Aus dem Institut für Diagnostische und Interventionelle Neuroradiologie

(Prof. Dr. med. M. Knauth)

der Medizinischen Fakultät der Universität Göttingen

\title{
Grey Matter Perfusion in Clinically Isolated Syndrome and Relapsing-Remitting Multiple Sclerosis
}

\author{
INAUGURAL - DISSERTATION \\ zur Erlangung des Doktorgrades \\ der Medizinischen Fakultät der \\ Georg-August-Universität zu Göttingen
}

vorgelegt von

Dr. rer. nat. Alina Freing

aus

Hamburg

Göttingen 2017 
Dekan:

Referent/in:

Ko-Referent/in:

Drittreferent/in:

Datum der mündlichen Püfung: 10.10.2017
Prof. Dr. rer. nat. H. K. Kroemer

Prof. Dr. med. M. Knauth

Prof. Dr. med. M. S. Weber

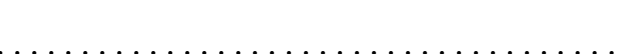


Hiermit erkläre ich, die Dissertation mit dem Titel „Grey Matter Perfusion in Clinically Isolated Syndrome and Relapsing-Remitting Multiple Sclerosis" eigenständig angefertigt und keine anderen als die von mir angegebenen Quellen und Hilfsmittel verwendet zu haben.

Göttingen, den 


\section{Contents}

List of Figures

$\begin{array}{ll}\text { List of Tables } & \text { IV }\end{array}$

\begin{tabular}{ll}
\hline List of Abbreviations & V
\end{tabular}

1 Introduction 1

1.1 Responsible Mechanisms . . . . . . . . . . . . . . . . . . . . . 3

1.2 Diagnostic Criteria and Clinical Appearance . . . . . . . . . . . . . . 5

1.3 Brain Atrophy as a Marker of Neurodegeneration . . . . . . . . . . . . . 7

1.4 Perfusion . . . . . . . . . . . . . . . . . . . . . . . . . . . . . . 9 9

2 Material and Methods 13

2.1 Magnetic Resonance Imaging . . . . . . . . . . . . . . . . . . . . . 13

2.2 Image Analysis . . . . . . . . . . . . . . . . . . . . . . . . . . . . . 14

$2.2 .1 \quad$ Brain Volumes . . . . . . . . . . . . . . . . . . . 15

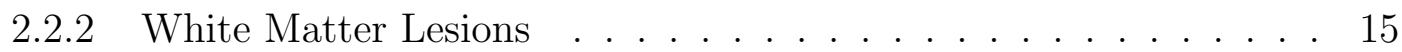

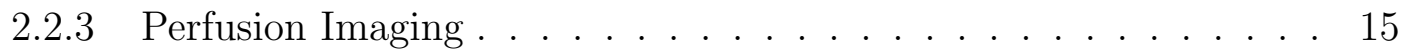

2.2 .4 Regions of Interest $\ldots \ldots \ldots$. . . . . . . . . . . . 16

2.3 Statistics . . . . . . . . . . . . . . . . . . . . . . . . . . 21

\begin{tabular}{lll}
\hline 3 & Results & 22 \\
\hline
\end{tabular}

3.1 Volumes . . . . . . . . . . . . . . . . . . . . . . . . . . . . . 22

3.2 Perfusion parameters $\ldots \ldots \ldots \ldots \ldots \ldots$. . . . . . . . . . . . . . . . 22

3.2 .1 Group Comparisons . . . . . . . . . . . . . . . . . . . 24

3.2 .2 Correlations . . . . . . . . . . . . . . . . . . . . 26 
$\begin{array}{lll}4 & \text { Discussion } & 35\end{array}$

4.1 Volumes $\ldots \ldots \ldots \ldots$

4.2 Perfusion parameters . . . . . . . . . . . . . . . . . . . . 37

4.2 .1 Group Comparisons . . . . . . . . . . . . . . . . . . . . . 38

4.2 .2 Correlations . . . . . . . . . . . . . . . . . . . . . . 41

4.2 .3 Responsible mechanisms . . . . . . . . . . . . . . . . . . 47

$4.3 \quad$ Conclusions and Outlook . . . . . . . . . . . . . . . . . . . . . . . . . . . . . . . . 49

$\begin{array}{lll}5 & \text { Summary } & 51\end{array}$

\begin{tabular}{lll}
\hline 6 & Bibliography & 53
\end{tabular} 


\section{List of Figures}

$2.1 \quad$ Exemplary arterial input function definitions. $\ldots \ldots \ldots$. . . . . . . 16

2.2 Normal plots of average relative cerebral blood volume values for each region of interest. . . . . . . . . . . . . . . . . . . . 17

2.2 Normal plots of average relative cerebral blood volume values for each region of interest. . . . . . . . . . . . . . . . . . . . . . . . . 18

2.3 Normal plots of average relative cerebral blood flow values for each region of interest. . . . . . . . . . . . . . . . . . . . . . . . . . . . . 19

2.3 Normal plots of average relative cerebral blood flow values for each region of interest . . . . . . . . . . . . . . . . . . . . . . . 20

$3.1 \quad$ Average relative cerebral blood volume and relative cerebral blood flow against normalized brain volume for all regions of interest. . . . . . . . . 28

3.2 Average relative cerebral blood volume and relative cerebral blood flow against Expanded Disability Status Scale for all regions of interest. . . . 29

3.3 Average relative cerebral blood volume and relative cerebral blood flow against disease duration for all regions of interest. . . . . . . . . . . . . 30

3.4 Boxplot for all regions of interest and all perfusion parameters. . . . . . . 31

$3.5 \quad$ Boxplot for intragroup comparison of perfusion parameters for all regions of interest. . . . . . . . . . . . . . . . . . . . . . . 32 


\section{List of Tables}

2.1 Cohort demographics. . . . . . . . . . . . . . . . . . 14

3.1 Comparison of the two different groups with respect to demographics. . . 23

3.2 Descriptive statistics for the different volumes derived from FreeSurfer. . 23

3.3 Descriptive statistics for relative cerebral blood volume. . . . . . . . . . . 24

3.4 Descriptive statistics for relative cerebral blood flow. . . . . . . . . . . . 25

3.5 Group comparisons with respect to relative cerebral blood volume. . . . . 31

3.6 Group comparisons with respect to relative cerebral blood flow. . . . . . 33

3.7 Multiple regression results for pooled data. . . . . . . . . . . . . . . . . . 33

3.8 Multiple regression results for subgroup of subjects diagnosed with clinically isolated syndrome. . . . . . . . . . . . . . . . . . . . 34

3.9 Multiple regression results for subgroup of subjects diagnosed with relapsing-remitting multiple sclerosis. . . . . . . . . . . . . . . . . . . 34 


\section{List of Abbreviations}

$\begin{array}{ll}\text { AIF } & \text { arterial input function } \\ \text { ASL } & \text { arterial spin labelling } \\ \text { CBF } & \text { cerebral blood flow } \\ \text { CBV } & \text { cerebral blood volume } \\ \text { CCSVI } & \text { chronic cerebrospinal venous insufficiency } \\ \text { CIS } & \text { clinically isolated syndrome } \\ \text { CNS } & \text { central nervous system } \\ \text { DisDur } & \text { disease duration } \\ \text { DSC } & \text { dynamic susceptibility contrast } \\ \text { EDSS } & \text { Expanded Disability Status Scale } \\ \text { ET-1 } & \text { endothelin-1 } \\ \text { gCIS } & \text { subgroup of subjects diagnosed with clinically isolated } \\ & \text { syndrome } \\ \text { Gd-enhancing lesions } & \text { Gadolinium-enhancing lesions on T1-weighted mag- } \\ & \text { netic resonance images } \\ \text { GM } & \text { cerebral grey matter } \\ \text { GMCort } & \text { region of interest comprising the cortical grey matter } \\ \text { gMS } & \text { subgroup of subjects diagnosed with relapsing- } \\ \text { IQR } & \text { remitting multiple sclerosis } \\ \text { MPRAGE } & \text { interquartile range } \\ \text { MRI } & \text { magnetization-prepared rapid acquisition and multi- } \\ \text { MS } & \text { magnetic resonance imaging } \\ & \text { multiple sclerosis } \\ & \end{array}$


MWU-Test

NAGM

NAWM

NBV

NGMV

NWMV

PPMS

PUT

$\mathrm{rCBF}$

$\mathrm{rCBV}$

ROI

RRMS

SPMS

$\mathrm{T} 2 \mathrm{w}$ lesions

T2wLES

THAL

WM

WMROI two-sided Mann-Whitney-U-Test

normal appearing cerebral grey matter

normal appearing cerebral white matter

normalized brain volume

normalized cerebral grey matter volume

normalized cerebral white matter volume

primary progressive multiple sclerosis

region of interest comprising the putamen

relative cerebral blood flow

relative cerebral blood volume

region of interest

relapsing-remitting multiple sclerosis

secondary progressive multiple sclerosis

hyperintense lesions on T2-weighted magnetic resonance images

region of interest comprising the hyperintense lesions on T2-weighted magnetic resonance images region of interest comprising the thalamus cerebral white matter region of interest comprising cerebral white matter 


\section{Introduction}

Multiple sclerosis (MS) is the most common acquired inflammatory demyelinating disorder of the central nervous system (Keegan and Noseworthy 2002) and it is the leading cause of non-traumatic neurological disability in young adults Compston and Coles 2002; Dutta and Trapp 2011). The clinical presentation of MS is diverse and ranges from focal neurological deficits to cognitive impairment. Radiologically, MS also presents a wide range of affections such as focal lesions in the sense of local inflammation and changes in diffusion and perfusion characteristics. The estimated prevalence of MS is about 2.5 million people worldwide (Noseworthy et al. 2000) and there is a notable gender imbalance, as two thirds to three quarters of the patients are women Noseworthy et al. 2000; Whitacre 2001). MS affects patients of all ages, but most patients, who present with a first manifestation of MS are aged between 20 and 50 years. MS was first described by Jean-Martin Charcot $(1825$ - 1893), who is often considered the founder of modern neurology. Charcot was the first to recognise MS as a distinct affliction and described it as the triad consisting of nystagmus, kinetic tremor and scanning speech. He also undertook the first neuropathological investigation of MS and linked his neuropathological findings to clinical symptoms. Even though there has been extensive research into the neurological, neuropathological and neuroradiological characteristics and mechanisms of MS since then, many aspects of MS remain elusive.

Familial genetic studies have shown that there is a genetic influence, as family members of MS patients have an up to 250-fold increased risk of developing MS compared to the average member of the public (Ebers et al. 2000). The individual genetic factor associated with the highest risk of developing MS is the HLA DRB1*15 variant of the human leukocyte antigen system, which codes for the MHC II-receptor Epplen et al. 1997; Compston 1999; Lin et al. 2012). This genetic variant increases the risk of developing MS by a factor of three and has been found in the more than $70 \%$ of 
MS patients (Epplen et al. 1997; Compston 1999; Lin et al. 2012). But even though there have been attempts to uncover the genetic risk factors for MS using genome-wide association studies, each respective identified risk factor on its own only accounted for a fractionally increased risk of developing the disease (Sadovnick et al. 1988; Robertson et al. 1996). Therefore the heritability of MS is thought to be polygenic and potentially even epigenetic.

The prevalence of MS is comparatively low in equatorial regions and increases towards both poles, constituting a distinct geographical pattern (Pugliatti et al. 2006). The reasons for this are unknown, but low vitamin $\mathrm{D}$ levels have been found to be a risk factor for acquiring MS, both in epidemiological studies (Munger et al. 2006) and in animal models of MS (Lemire and Archer 1991; Cantorna et al. 1996; Spach and Hayes 2005).

The Epstein-Barr virus has received special attention with respect to the risk of acquiring MS. Avoiding Epstein-Barr virus infection significantly reduces the individual risk of developing MS in adults (Sumaya et al. 1985; Munch et al. 1997; Myhr et al. 1998; Ascherio and Munch 2000; Wagner et al. 2000; Ascherio et al. 2001; Haahr et al. 2004; Sundström et al. 2004; Ponsonby et al. 2005) as well as in children Alotaibi et al. 2004; Pohl et al. 2006). Additionally, there is also a significant difference with respect to the risk of acquiring MS between seropositive individuals with and without a history of infectious mononucleosis (Thacker et al. 2006), which in the opinion of Ascherio and Munger (2007a) suggests that older age at infection further increases the odds of developing MS. The significance of infection with the Epstein-Barr virus has been controversially discussed because of the general prevalence of Epstein-Barr antibodies of 95\% in adults, which makes it difficult to analyse the suspected effect to any sufficient degree of certainty. But as Ascherio and Munger (2007a) pointed out, there are other viruses such as the polio virus, which show similar behaviour: the polio virus used to be endemic in some countries, infecting virtually all children, but only causing poliomyelitis in a limited number of cases. So the high seroprevalence of the Epstein-Barr virus is not in itself a valid argument for discounting it as a potentially important player in the acquisition of MS (Ascherio and Munger 2007a).

Several other influencing factors have been identified or speculated upon. Smoking has been identified both, as a general risk factor and also as interacting negatively with the risk posed by an Epstein-Barr virus infection (Simon et al. 2010). Other virus infections 
than Epstein-Barr virus have been discussed as potentially increasing or decreasing the risk of developing MS, depending on the virus (reviewed in Bach 2002). However, it should be noted that most studies used animal models of autoimmune disease. Since there is a notable gender imbalance and also a typical age range, gender and age seem to be influential factors. Mandoj et al. (2015) found a disrupted lipid homeostasis was associated with high disease activity, while Fellows et al. (2015) found high serum levels of HDL-cholesterol to be protective with respect to the integrity of the blood brain barrier in MS patients. Potentially influential factors such as hormons, especially estrogens, diet, both with respect to fatty acids and with respect to antioxidants have been discussed (see Ascherio and Munger (2007b) for a comprehensive review). General hygiene has received some attention with respect to developing autoimmune diseases in general, not only MS. But the hypothesis of Leibowitz et al. (1966), which postulates that exposure to several infectious agents early in life is protective against MS is still a topic of scientific discussion.

\subsection{Responsible Mechanisms}

Despite its common occurrence and years of scientific effort, the mechanisms of MS are still comparatively poorly understood. However, it is generally accepted that there are two major components in MS, namely neuroinflammation and neurodegeneration. The aspect that has long been in the focus of attention is the (focal) inflammatory component. The reasons for this central role mostly stem from history. For one thing, focal brain lesions were the first affection of MS to be detected early on in brain dissection of MS patients. White matter brain lesions were also the first MS affection, which could be detected and quantified in vivo, when magnetic resonance imaging (MRI) first became available. Additionally, there is a significant amount of pharmaceutical knowledge about targeting and regulation parts of the immune system, which has been around for a while. So researching the inflammatory component held much more promise in terms of potential drug development.

The inflammatory component of MS is thought to be mediated by autoreactive T- and B-cells, which migrate to the central nervous system causing focal demyelination, oligodendrocyte loss and also neuronal damage (Dutta and Trapp 2007; Trapp and Nave 2008; Dutta and Trapp 2011; Kipp et al. 2012). Infiltrating macrophages were found 
in single large-mass (tumefactive) lesions alongside myelin loss and preserved axons in clinically isolated syndrome (CIS) patients (Miller et al. 2005b). However, it should be kept in mind that these histopathological findings are based on atypical presentations of CIS, which makes them inherently biased. Furthermore, primary mechanisms such as oligodendrocyte dysfunction have also been found to be a potential diseasetriggering factor (Barnett and Prineas 2004). These changes are not limited to focal lesions, however (Bö et al. 2006). Similar changes have been found in extensive areas of seemingly normal white matter (Kutzelnigg et al. 2005, Androdias et al. 2010). Recent histopathological and MRI findings have shown that MS pathology also involves grey matter lesions and diffuse grey matter damage (Bö et al. 2006; Filippi et al. 2012; Kipp et al. 2012), where focal grey matter lesions also show demyelination and oligodendrocyte loss (Bö et al. 2006; Kipp et al. 2012). Lucchinetti et al. (2011) even found inflammatory meningeal pathology additional to widespread cortical pathology outside of lesions.

The second major component is neurodegeneration. Its pathogenesis is less well understood than that of neuroinflammation. Axonal pathology in MS lesions and its mechanisms have received much attention (Ferguson et al. 1997; Trapp et al. 1998; Kornek et al. 2000; Bjartmar et al. 2000; Kuhlmann et al. 2002). Cortical lesions are a common occurence in MS patients and have been found in $26 \%$ of MS patients by means of in vivo MRI, as well as post mortem MRI and neuropathological analysis (Kidd et al. 1999). While these focal grey matter lesions display inflammatory features, they also display substantial amounts of axonal and neuronal damage (Bö et al. 2006; Kipp et al. 2012). Peterson et al. (2001) also found death of neuronal cell bodies early on in the disease in a post mortem tissue analysis of cortical lesions in MS patients. Sailer et al. (2003) and Inglese et al. (2004) also found widespread grey matter involvement in normal appearing cerebral grey matter (NAGM) and focal cortical thinning in an in vivo MRI study, suggesting axonal loss.

However, neuroinflammation and neurodegeneration are not two separate components, but are intrinsically linked to each other. There is evidence that the destruction in focal inflammatory MS lesions leads to Wallerian degeneration, which in turn contributes significantly to early axonal pathology in MS patients Casanova et al. 2003; Dziedzic et al. 2010). Wallerian degeneration has also been suggested as the process responsible for diffuse tissue damage in the so called normal appearing cerebral white matter 
(NAWM) outside focal lesions (Seewann et al. 2009). Trapp et al. (1998) also found that early axonal pathology correlates with immune cell infiltration. Conversely, Wallerian degeneration has also been implicated in the recruitment of inflammatory cells into the central nervous system (CNS) (Tsunoda et al. 2007).

\subsection{Diagnostic Criteria and Clinical Appearance}

There is no such thing as "the" MS. Rather, MS is a collection of several different subtypes, which are characterised by markedly different forms of progression. There is a wide range from very benign courses to rapidly-progressing disability. Moreover, the subtype is not definite and can change in the course of the disease. Lublin and Reingold (1996) defined the following three subtypes and revised them in 2013 Lublin et al. 2014):

- CIS: clinical presentation of a disease which shows characteristics of inflammatory demyelination and which could be MS, but does not yet fulfil the diagnostic criteria of MS with respect to dissemination in time.

- Relapsing-remitting multiple sclerosis (RRMS): clearly defined disease relapses with full recovery or a residual neurological and/or functional deficit. The periods between relapses are characterised by a lack of disease progression.

- Primary progressive multiple sclerosis (PPMS): disease progression from onset with occasional plateaus. Temporary minor improvements are allowed, but distinct relapses are not.

- Secondary progressive multiple sclerosis (SPMS): initially shows all characteristics of RRMS, but turns into progression which might be overlaid with additional relapses. Minor remissions and plateaus are possible, but if there are still relapses, periods between those relapses are characterised by disease progression (as opposed to RRMS).

McDonald et al. (2001) first developed objective criteria for definitely diagnosing MS. These criteria have since been revised by the International Panel on Diagnosis of MS (Polman et al. 2011). It should be noted that these criteria are explicitly developed 
and validated for patients who present with a typical CIS suggestive of RRMS or at least with symptoms consistent with inflammatory demyelinating processes in the CNS and should not be applied to different collectives (Polman et al. 2011). The diagnosis of MS is the result of a synthesis of clinical and especially MRI-derived paraclinical information (Polman et al. 2011). Beyond symptoms of inflammatory demyelination of the CNS in the sense of hyperintense lesions on T2-weighted magnetic resonance images (T2w lesions) and Gadolinium-enhancing lesions on T1-weighted magnetic resonance images (Gd-enhancing lesions), it essentially requires the exclusion of other disorders that can mimic MS and objective evidence for a disseminated disease course both in time and in space. The dissemination can either be substantiated clinically or by means of MRI according to specific criteria (Swanton et al. 2006, 2007; Polman et al. 2011). Additionally, MRI has developed into the primary tool to distinguish CIS from early MS (McDonald et al. 2001; Rot and Mesec 2006; Swanton et al. 2007; Miller et al. 2008; Polman et al. 2011). For the sake of completeness, it should be noted that the European collaborative research network that studies magnetic resonance imaging in multiple sclerosis (MAGNIMS) has recently suggested several modifications (Filippi et al. 2016) to the 2010 revisions of the McDonald criteria (Polman et al. 2011) with regard to MRI.

The clinical appearance of MS is heterogeneous, depending on the anatomical areas targeted by intense disease activity. Patients presenting with CIS typically show symptoms linked to the optic nerve, the brain stem/cerebellum, the spinal cord, or the cerebral hemispheres (Polman et al. 2011). About $85 \%$ of patients who later develop RRMS first present with a clinically isolated syndrome (Miller et al. 2005b), but there is no pathognomonic characteristic to predict which of the CIS patients will develop definite RRMS. Generally, more than $80 \%$ of the MS patients start out with CIS or RRMS, but convert to SPMS approximately 20 years after onset (Confavreux and Vukusic 2006). In RRMS there is a good correlation between new Gd-enhancing lesions and the occurrence of relapses, which brakes down when the disease converts to a progressive form (Pittock et al. 2004). The inflammatory processes corresponding to such a Gadolinium uptake generally cause focal inflictions such as optic neuritis, locally limited dysesthesia or reduced strength in limited areas. In contrast to this, diffuse axonal damage often leads to neurological and cognitive impairments (Keegan and Noseworthy 2002; Schulz et al. 2006). Cognitive impairment is generally a common occurrence in MS and affects 
$43-70 \%$ of the patients (Rao et al. 1991; Amato et al. 2001; Chiaravalloti and DeLuca 2008). It can be detected already comparatively early in the disease course (Amato et al. 1995) and has a considerable impact on both, the private and the working life of MS patients (Rao et al. 1991; Chiaravalloti and DeLuca 2008). Additionally, MS patients show as much as a tenfold increase in frequency of epileptic seizures compared to healthy subjects (Eriksson et al. 2002).

Therapeutic options in MS are limited and directly depend on the clinical subtype as introduced above. There is a reasonable range of immunomodulatory drugs available, which target the neuroinflammatory component of RRMS. Several of these diseasemodifying agents verifiably reduce the number of relapses, MRI-derived disease activity and to a lesser degree even the progression of clinical disability in RRMS (e.g. Polman et al. 2006; Mikol et al. 2008; Kappos et al. 2010; Cohen et al. 2012; Gold et al. 2012; Calabresi et al. 2014). However, therapeutic options are rare with respect to the progressive forms of MS presumably dominated by neurodegeneration. These immunomodulatory agents which are effective in RRMS only have a very limited effect in progressive forms of MS (European Study Group on Interferon $\beta$-1b in Secondary Progressive MS 1998; Leary and Thompson 2003). Mitoxantrone is used in SPMS, but its use is limited by severe cumulative side effects. There is currently no therapeutic agent approved for use in PPMS (Ransohoff et al. 2015). Treatment decisions in PPMS are made on an individual basis (Ontaneda et al. 2015). In light of this, it is even more important that several clinical trials have shown that administering disease-modifying treatment to CIS patients reduces their likelihood of developing RRMS and also reduces MRI-derived disease activity (Jacobs et al. 2000; Comi et al. 2001; Kappos et al. 2006; Comi et al. 2009). Similarly, transition to SPMS can be delayed by effective immunomodulatory medication (Tedeholm et al. 2013). Therefore timely treatment is also of prognostic importance on several counts.

\subsection{Brain Atrophy as a Marker of Neurodegenera- tion}

Conventional MRI with contrast enhancement is a routine means of diagnosing and monitoring the course of MS. But even if the current diagnostic criteria (Polman et al. 
2011; Filippi et al. 2016) only consider T2w lesions and Gd-enhancing lesions, there is far more information which MRI can provide in the context of MS. This is of particular importance as the correlation between conventional radiological parameters and clinical disability is comparatively poor (Barkhof 2002).

One of the parameters on which MRI can provide information is brain atrophy. Reliable methods to estimate brain atrophy have been around for some time (Bermel and Bakshi 2006; Zipp 2009) and are constantly being refined (Smeets et al. 2016). There is a significantly increased annual brain volume loss of $0.5-1.0 \%$ in MS patients compared to an annual brain volume loss of $0.1-0.3 \%$ in healthy individuals (Simon 2006; Fotenos et al. 2008; Fisher et al. 2008; Barkhof et al. 2009; De Stefano et al. 2010; De Stefano et al. 2014, Vollmer et al. 2015). This brain volume loss occurs in all MS patients regardless of the respective subtype (Tedeschi et al. 2005, De Stefano et al. 2010). Both, demyelination due to inflammatory activity and neurodegeneration contribute to this loss (Simon 2006; Barkhof et al. 2009; Barten et al. 2010). Contrary to longheld beliefs, neurodegeneration begins early on in the disease (Silber and Sharief 1999; Dutta and Trapp 2007) and is already visible on MRI of CIS patients suggestive of MS (Chard et al. 2002; Henry et al. 2008; Chard and Miller 2009; Raz et al. 2010). There is a correlation between brain volume loss and disability/disease progression (Bermel and Bakshi 2006; Simon 2006; Amato et al. 2007; Minneboo et al. 2008; Fisniku et al. 2009; Lukas et al. 2010; Filippi and Rocca 2011; Zivadinov et al. 2013b; De Stefano et al. 2014; Jacobsen et al. 2014) and Sormani et al. (2014) showed that additionally measuring brain atrophy provides new information in comparison with just quantifying $\mathrm{T} 2 \mathrm{w}$ lesion volume. Today, measurement of brain volume change is widely accepted as a method for quantifying neurodegeneration and is recommended for use in clinical trials to assess neurodegeneration, neuroprotection and the efficacy of tested therapies (e.g. Zivadinov and Bakshi 2004; Barkhof et al. 2009; De Stefano et al. 2014). Some of the available software tools such as SIENAX (Smith et al. 2002; Smith et al. 2004) and FreeSurfer (Fischl et al. 2002) also provide estimates of regional volumes. This addresses the fact that several studies suggest that it is mainly grey matter atrophy, which predicts disease progression, disability and cognitive impairment (De Stefano et al. 2003; Amato et al. 2004; Chard et al. 2004; Sanfilipo et al. 2005; Sanfilipo et al. 2006; Fisher et al. 2008; Zivadinov et al. 2013b; Popescu et al. 2013; Zivadinov et al. 2013a; Fisniku et al. 2008). As De Stefano et al. (2014) pointed out, this may have important implications 
with respect to the recommendation to use brain atrophy as a primary end-point for measuring neuroprotection in clinical trials. Furthermore, neurodegeneration in the form of MRI-derived brain atrophy also has some prognostic value with respect to the conversion from CIS to MS (Bjartmar et al. 2000). But on a note of caution, it should be mentioned that there are several confounders such as image quality, age, life habits, genetic load and comorbidities (Enzinger et al. 2005; Zivadinov et al. 2009; De Stefano et al. 2014), which can obscure the underlying processes.

\subsection{Perfusion}

Changes in perfusion in MS patients have received increasing scientific attention in the last couple of years. Early on, Wuerfel et al. (2004) showed that the inflammatory processes involved in forming a new MS plaque are accompanied by altered local perfusion. This change in local perfusion can be detected by means of perfusion MRI prior to permeability of the blood brain barrier (Wuerfel et al. 2004). Wuerfel et al. (2004) concluded that elevation of perfusion must therefore be an early event in the development of a plaque. This is in line with the results of Haselhorst et al. (2000) and Ge et al. (2005b), who also found that the early stage of plaque development is characterised by inflammation and increased perfusion. Contrarily, normal to diminished blood supply has been described for fully-formed MS plaques with a tendency to decrease further with increasing axonal damage (Haselhorst et al. 2000; Law et al. 2004; Ge et al. 2005b). Several other studies suggest that overall perfusion of MS patients in lesions as well as in various parts of NAWM and NAGM is decreased compared to healthy subjects (Law et al. 2004; Ge et al. 2005b; Adhya et al. 2006; Inglese et al. 2007). It is therefore a broad consensus that MS patients display altered cerebral perfusion.

An idea which has been the center of intense and controversial discussion is the concept of venous drainage pathology termed chronic cerebrospinal venous insufficiency (CCSVI), which was reintroduced by Zamboni $(2006)$ as a potential reason for developing MS. Professor Zamboni has suggested that endovascular therapy of the vein blocking the cranial outflow might be a means to cure MS and his group has published several studies in support of the CCSVI theory (Zamboni et al. 2007; Zamboni et al. 2009a; Zamboni et al. 2009b; Zamboni et al. 2009c; Bartolomei et al. 2010; Zamboni and Galeotti 2010). However, the study of Zamboni (2006) has been severely critizised with 
respect to the methods and criteria implied, as comprehensively presented by Valdueza et al. (2013). Most other research groups could not corroborate that cerebral venous pathology occurs more often in MS than in healthy controls, neither using ultrasound nor the more objective MRI (e.g. Sundström et al. 2010; Doepp et al. 2010; Doepp et al. 2011; Wattjes et al. 2011b; Wattjes et al. 2011a; Zivadinov et al. 2011; Bourdette and Cohen 2014; Tsivgoulis et al. 2015; Krogias et al. 2016; Cardaioli et al.|2016).

But the general idea that there is some sort of MS-associated vasculopathy is still around, as small venules have been found to be affected in MS in several ways. Histopathological studies found significant wall damage and perivascular inflammation (Tanaka et al. 1975; Adams et al. 1985, Adams 1988). These findings were corroborated by MRI, which showed widespread perivascular inflammation and altered perivascular spaces (Ge et al. 2005a; Wuerfel et al. 2008). High field MRI could also show that each MS plaque is associated with a venule (Tallantyre et al. 2008; Ge et al. 2008; Kollia et al. 2009; Tallantyre et al. 2009; Tallantyre et al. 2011; Sinnecker et al. 2012a; Sinnecker et al. 2012b; Wuerfel et al. 2012). Furthermore, Sinnecker et al. (2013) found that the general density of perivascular veins is reduced in MS. They attribute this rarefication in part to venous pathology (Sinnecker et al. 2013). The perivascular configuration of MS plaques and the rarefication of perivascular veins has given rise to the idea that obliterating processes in the course of lesion chronification might be one of the reasons for reduced cerebral perfusion in MS patients. But D'haeseleer et al. (2015) convincingly argue against this hypothesis by pointing out that this would lead to a more patchy pattern of focal cerebral blood flow $(\mathrm{CBF})$ decrease than that observed in MS patients. Similarly, there is no indication of substantial obliteration such as microvessel thrombosis and other structural abnormalities in focal MS lesions (De Keyser et al. 2008), which also argues against obliterating processes being the reason for hemodynamic changes.

Another mechanism which has been suggested as a reason for reduced cerebral perfusion is an altered metabolism. Blood supply in the brain is regulated by astrocytes according to blood and oxygen demands of the local neurons (Petzold and Murthy 2011). While a decrease in cerebral perfusion in this context seems a somewhat natural consequence of axonal loss and the implied reduction in metabolism, there are several studies which point to other pathomechanisms. For one thing, there is an increase in the excitability of primary motor cortex neurons of MS patients, which suggests an increase of metabolism (Dutta et al. 2006; Conte et al. 2009; Vucic et al. 2012). Furthermore, Debernard et al. 
(2014) showed that reduced grey matter perfusion is already present in RRMS patients who do not show pathological brain volume loss yet. This indicates that axonal loss is probably not the driving mechanism of cerebral hypoperfusion (D'haeseleer et al. 2015). This is consistent with the findings of Saindane et al. (2007), who simultaneously used perfusion and diffusion tensor imaging to investigate hemodynamic changes. They found the decreased $\mathrm{CBF}$ levels in their study to be rather consistent with primary ischemia than with hypoperfusion caused by Wallerian degeneration (Saindane et al. 2007). This is in line with the results of De Keyser et al. (2008), who found that ischemic changes are a possible reason for lesion development in certain types of lesions instead of a consequence (De Keyser et al. 2008). The notion that decreased perfusion in MS patients is not simply a direct consequence of a reduced metabolism is further supported by the spectroscopic results of Steen et al. (2013).

Beyond an altered metabolism, primary astrocyte dysfunction has been suggested as a key player in the pathomechanism of reduced perfusion De Keyser et al. (2008). Reactive astrocytes in MS plaques have also been identified as a possible source for elevated levels of the vasoconstrictive peptide endothelin-1 (ET-1) (D'haeseleer et al. 2013), which has recently become the focus of scientific attention. Several studies have found enhanced blood levels of ET-1 in peripheral venous blood as well as in cerebrospinal fluid of MS patients (Speciale et al. 2000; Haufschild et al. 2001; D'haeseleer et al. 2013). Enhanced blood levels of ET-1 have also been associated with reduced extra-ocular blood flow velocities (Pache et al. 2003). Furthermore, Marshall et al. (2014) could show that the functional response of cerebral arterioles to vasomotor stimulation is impaired in MS patients, which indicates the presence of counteracting vasoconstrictive effects. In addition to that, D'haeseleer et al. (2013) even found that reduced perfusion in MS patients could be significantly increased by administrating an ET-1 receptor antagonist. Overall, these findings provide strong evidence that reduced cerebral perfusion in MS patients is at least partly mediated by elevated ET-1 levels, suggesting a pathology of arterioles as an important player in hemodynamic change in MS patients.

Impaired perfusion in MS patients has been linked to loss to higher brain functions, such as verbal memory and executive motor task function, as well as to fatigue score (Inglese et al. 2007; Papadaki et al. 2012; Papadaki et al. 2014a; Papadaki et al. 2014b). This assumes a new significance in light of the emerging understanding that impaired perfusion in MS is not simply a consequence of inflammation and/or axonal loss, but 
might independently contribute to disease progression and all the ensuing disability. This makes cerebral perfusion a valuable tool not only in assessing disease progressing, but also in assessing the efficacy of drugs undergoing clinical testing. Moreover, hemodynamic change is a process, which starts early on in the disease (e.g. Rashid et al. 2004; Papadaki et al. 2012; Papadaki et al. 2014b). Also, Varga et al. (2009) found some evidence that hemodynamic change in MS starts out in the NAWM and only spreads to the cerebral grey matter (GM) as the disease progresses. Considering this in view of the potentially reversible nature of this impaired perfusion D'haeseleer et al. (2013), restoring cerebral perfusion could become an important pharmacological target in the hitherto inadequately addressed diffuse axonal damage in MS. It is therefore crucial to further investigate the process underlying impaired cerebral perfusion in MS to better understand its (temporal) evolution and the mechanisms governing it. 


\section{Material and Methods}

A total of 106 untreated patients aged between 18 and 65, who had been diagnosed with CIS or RRMS according to the revised McDonald criteria (Polman et al. 2011) were prospectively recruited from the Charité outpatient clinic, Department of Neurology, Charité University Medicine, Berlin, Germany. A total of 11 patients were excluded, 3 because the diagnosis was revised in the course of the study, 5 because of technical problems (contrast agent peak not properly captured, motion artefacts), 2 because of insufficient enhancement and 1 because of an unusually shaped lesion suspective of a tumour. The remaining 95 patients were included in this study.

All patients underwent a neurological examination conducted by a board-certified neurologist and were evaluated according to the EDSS (Kurtzke 1983). Written informed consent according to the declaration of Helsinki was obtained from all patients. A positive vote of the Ethics Committee at Charité University Medicine, Berlin, Germany was obtained (EA1/182/10). More detailed demographic information can be found in Table 2.1.

\subsection{Magnetic Resonance Imaging}

All subjects underwent MRI. The following sequences were acquired in all subjects on a $3 \mathrm{~T}$ scanner (Tim Trio, Siemens Medical Systems, Erlangen, Germany) at the Berlin Center of Advanced Neuroimaging (Charité University Medicine, Berlin, Germany):

1. Three-dimensional T1- weighted $1 \times 1 \times 1 \mathrm{~mm}^{3}$ isotropic magnetization-prepared rapid acquisition and multiple gradient echo technique (MPRAGE), TE $2.55 \mathrm{~ms}$, TR $1900 \mathrm{~ms}$, TI $900 \mathrm{~ms}$.

2. Three-dimensional T2-weighted $1 \times 1 \times 1 \mathrm{~mm}^{3}$ isotropic, TE $502 \mathrm{~ms}$, TR $5000 \mathrm{~ms}$. 


\begin{tabular}{lll}
\hline Subjects & $\mathrm{n}$ & 95 \\
\hline Gender & Male & $29(31 \%)$ \\
& Female & $66(69 \%)$ \\
Age (years) & Mean \pm SD & $32 \pm 9$ \\
& Min $-\max$ & $18-56$ \\
EDSS & Median & 1.5 \\
& Min $-\max$ & $1-4$ \\
NBV $\left(\mathrm{cm}^{3}\right)$ & Mean \pm SD & $1707 \pm 91$ \\
& Min $-\max$ & $1451-1973$ \\
NGMV $\left(\mathrm{cm}^{3}\right)$ & Mean \pm SD & $692 \pm 38$ \\
& Min $-\max$ & $590-804$ \\
NWMV $\left(\mathrm{cm}^{3}\right)$ & Mean $\pm \mathrm{SD}$ & $1015 \pm 58$ \\
& Min $-\max$ & $862-1168$ \\
\hline
\end{tabular}

Table 2.1: Cohort demographics. SD: standard deviation; Min: minimum value; max: maximum value.

3. Three-dimensional T2-weighted fluid-attenuated inversion recovery $1 \times 1 \times 1 \mathrm{~mm}^{3}$ isotropic, TE $388 \mathrm{~ms}$, TR $5000 \mathrm{~ms}$, TI $1800 \mathrm{~ms}$.

4. Two-dimensional dynamic susceptibility contrast (DSC) echo-planar imaging $1.8 \times 1.8 \mathrm{~mm}^{2}$ with a slice thickness of $5 \mathrm{~mm}$ (TE $30 \mathrm{~ms}$, TR $1490 \mathrm{~ms}$ ) after intravenous injection of $7 \mathrm{ml}$ Gadovist $(0.1 \mathrm{mmol} / \mathrm{kg}$ body weight, Bayer Healthcare Germany, Radiology, Leverkusen) at a rate of $3 \mathrm{ml} / \mathrm{s}$ followed by $20 \mathrm{ml}$ saline. Data acquisition started $10 \mathrm{~s}$ before the beginning of the contrast agent injection with a temporal resolution of $1.5 \mathrm{~s}$ and was continued for $75 \mathrm{~s}$.

\section{$2.2 \quad$ Image Analysis}

All acquired images were stored in DICOM file format in an instance of version 1.6.5 of the open-source Extensible Neuroimaging Archive Toolkit (Marcus et al. 2007), which is available for download at http://www.xnat.org/download. Most steps of the image analysis was implemented as a pipeline in the framework provided by the Extensible Neuroimaging Archive Toolkit. All computations were carried out on a Mac Mini (OS X 10.9.5, Intel Core i7 2.6 GHz, 16 GB 1600 MHz DDR3). 


\subsubsection{Brain Volumes}

Brain tissue volume, normalised for subject head size, was estimated with version 2.6 of SIENAX (Smith et al. 2001, 2002), part of FSL (Smith et al. 2004), which is available for download at https://fsl.fmrib.ox.ac.uk/fsldownloads/fsldownloadmain.html. SIENAX starts by extracting brain and skull images from the single whole-head input data (Smith 2002). The brain image is then affine-registered to MNI152 space (Jenkinson and Smith 2001; Jenkinson et al. 2002), using the skull image to determine the registration scaling. The registration is primarily carried out to obtain the volumetric scaling factor, which is used as a normalisation for head size. In the next step, tissue-type segmentation with partial volume estimation is carried out (Zhang et al. 2001) in order to calculate the normalised total volume of brain tissue, including separate estimates of the volumes of grey matter and white matter. SIENAX is referenced here as requested in the documentation (SIENAX 2016).

\subsubsection{White Matter Lesions}

Bulk white matter T2w lesion load was manually quantified on T2-weighted sequences using version 6.0 of the OsiriX open-source software (Rosset et al. 2004), which is available for download at http://www.osirix-viewer.com. Binary lesion masks were created and converted to NIfTI file format. 11 patients did not show any white matter lesions.

\subsubsection{Perfusion Imaging}

Perfusion images were analysed version 6.0 of the OsiriX open-source software (Rosset et al. 2004) and the IB Neuro (Imaging Biometrics, LLC, http://www. imagingbiometrics.com, v1.2) plug-in, which offers good accuracy and consistency (Hu et al. 2015). The IB Neuro plug-in offers standardised rCBF maps and rCBV maps corrected for contrast agent leakage. Standardization is carried out by transforming rCBV values to a consistent intensity scale regardless of make, model and field strength of the scanner used (Schmainda et al. 2004; Boxerman et al. 2006; Bedekar et al. 2010). In order to define the AIF, four voxels were manually selected in distant branches of the medial cerebral artery (Ebinger et al. 2010) with early and well-defined contrast agent 


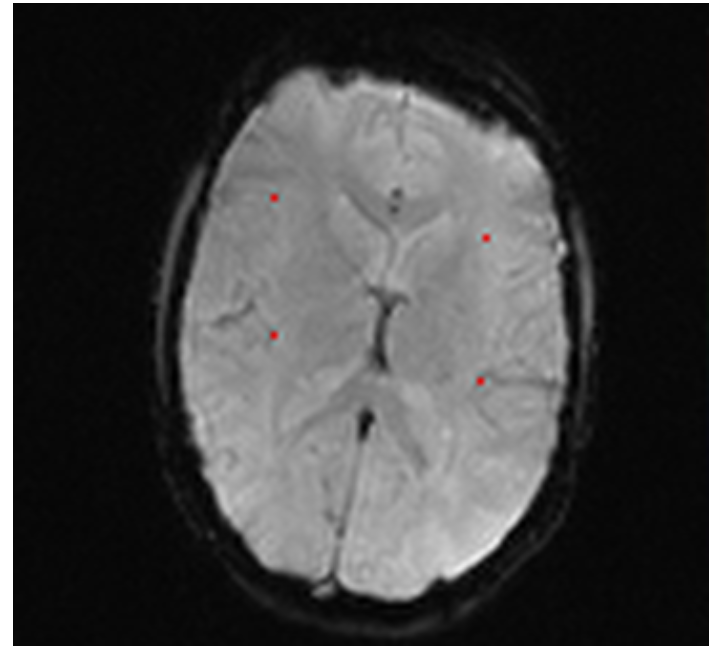

(a) Defining voxels for AIFs.

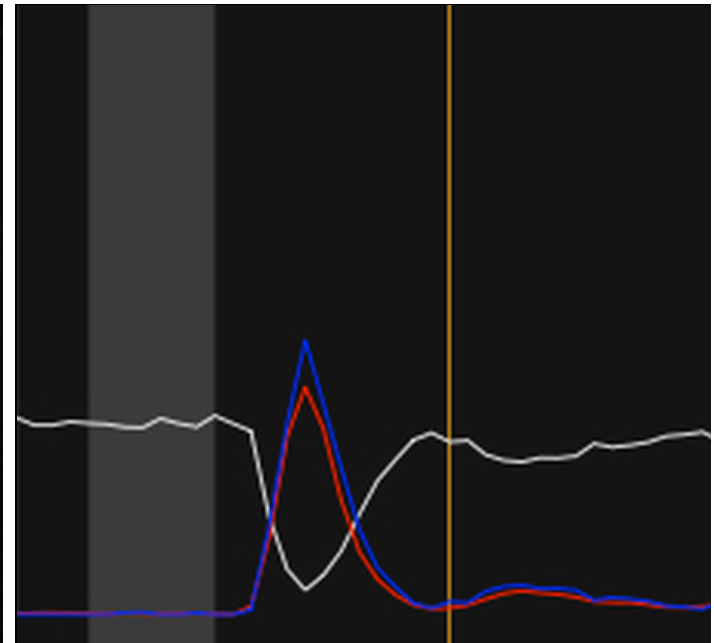

(b) Defining voxels for AIFs.

Figure 2.1: (a) Exemplary defining voxels for AIF and (b) resulting curves (last manually defined AIF in white, average AIF in red, difference between last manually defined AIF and average AIF in blue). Light gray interval denotes definition of the baseline, vertical orange line denotes the end of the integration interval.

inflow, two in each hemisphere (see Figure 2.1 for an example). All created maps were converted to NIfTI file format.

\subsubsection{Regions of Interest}

Volumetric segmentation was performed with version 5.2.0 of FreeSurfer, which is documented and available for download at http://surfer.nmr.mgh.harvard.edu. Details on FreeSurfer processes are described in more detail in Dale and Sereno (1993), Dale et al. (1999), Fischl et al. (1999a), Fischl et al. (1999b), Fischl and Dale (2000), Fischl et al. (2001), Fischl et al. (2002), Fischl et al. (2004a), Fischl et al. (2004b), Ségonne et al. (2004), Han et al. (2006), Jovicich et al. (2006), Reuter et al. (2010) and Reuter et al. (2012). Briefly, this processing includes motion correction and averaging of multiple volumetric T1 weighted images, if more than one is available (Reuter et al. 2010), removal of non-brain tissue using a hybrid watershed/surface deformation procedure (Ségonne et al. 2004), automated Talairach transformation, segmentation of the subcortical white matter and deep grey matter volumetric structures including thalamus, hippocampus, amygdala, caudate, putamen, ventricles (Fischl et al. 2002, 2004a), in- 


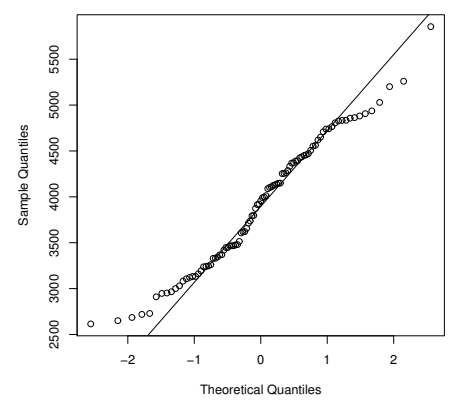

(a) $\mathrm{rCBV}$ (WMROI)

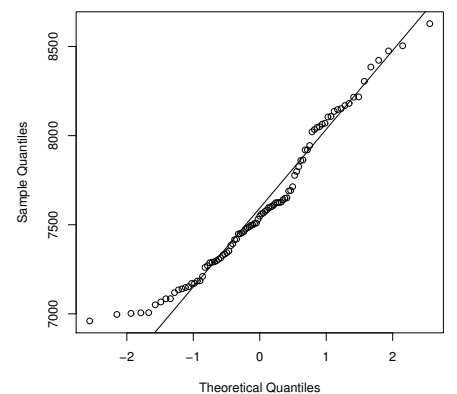

(d) rCBV (GMCort)

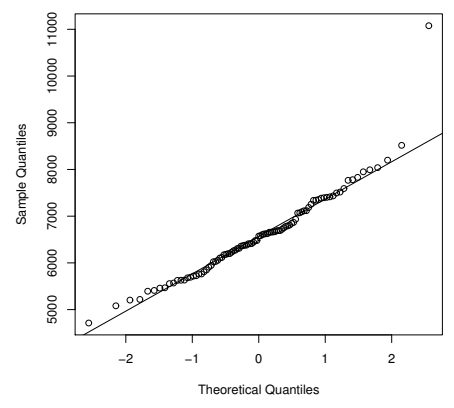

(g) rCBV (THAL)

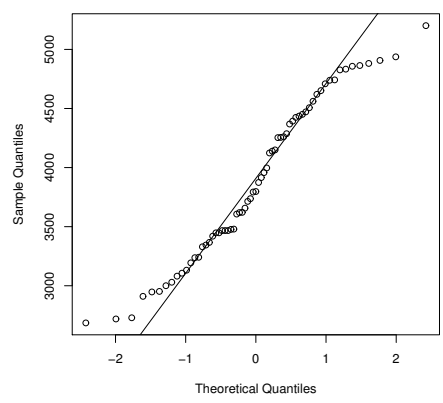

(b) rCBV (WMROI, CIS)

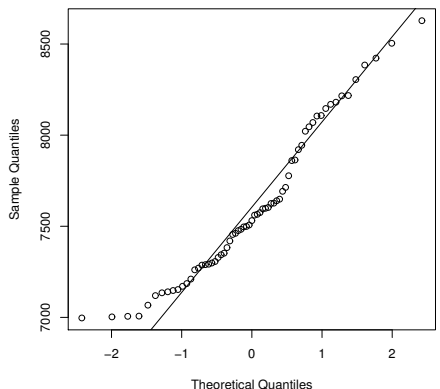

(e) rCBV (GMCort, CIS)

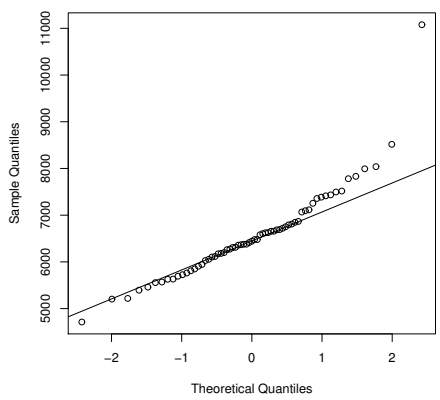

(h) rCBV (THAL, CIS)

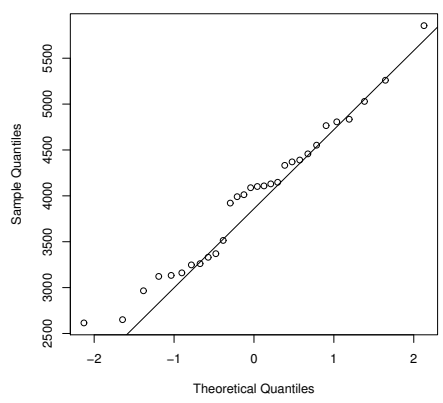

(c) rCBV (WMROI, MS)

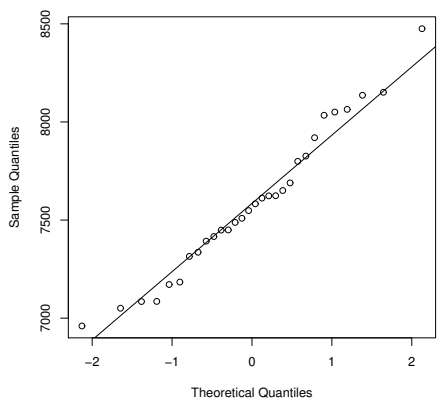

(f) rCBV (GMCort, MS)

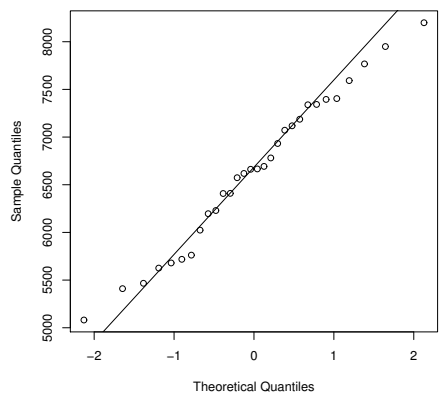

(i) $\mathrm{rCBV}$ (THAL, MS)

Figure 2.2: Normal plots of average rCBV values for each ROI and the respective patient groups (all, CIS, MS).

tensity normalization (Sled et al. 1998), tessellation of the grey matter white matter boundary, automated topology correction (Fischl et al. 2001; Ségonne et al. 2007) and surface deformation following intensity gradients to optimally place the grey/white and grey/cerebrospinal fluid borders at the location where the greatest shift in intensity defines the transition to the other tissue class (Dale et al. 1999; Dale and Sereno 1993; 


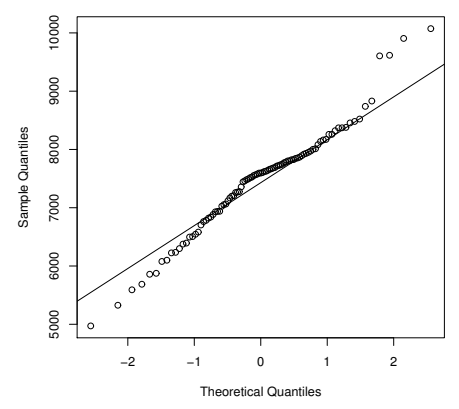

(j) $\mathrm{rCBV}(\mathrm{PUT})$

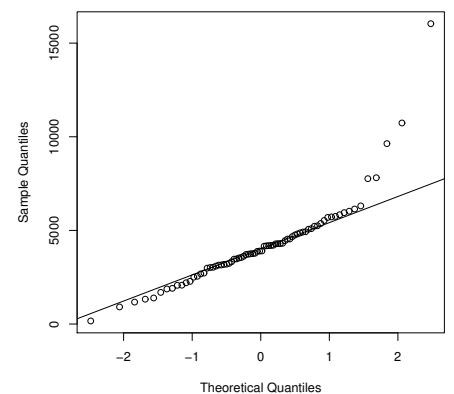

(m) rCBV (T2wLES)

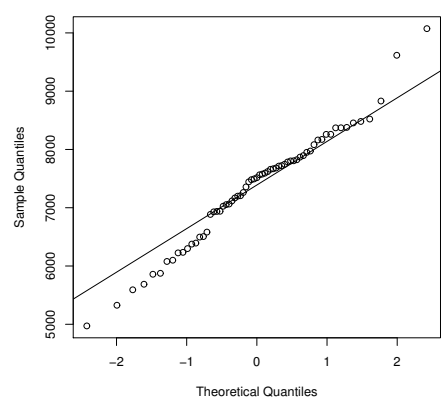

(k) $\mathrm{rCBV}(\mathrm{PUT}, \mathrm{CIS})$

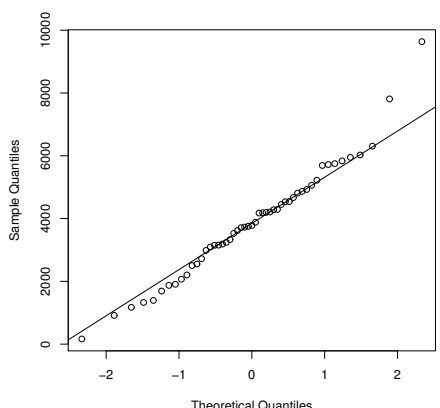

(n) rCBV (T2wLES, CIS)

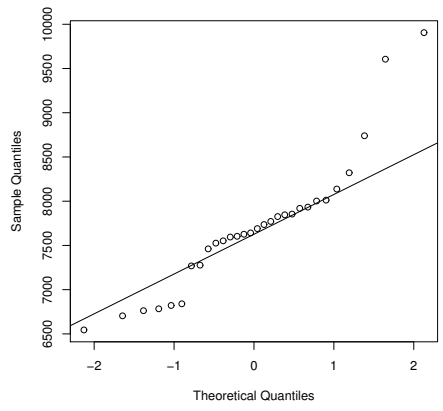

(l) $\mathrm{rCBV}(\mathrm{PUT}, \mathrm{MS})$

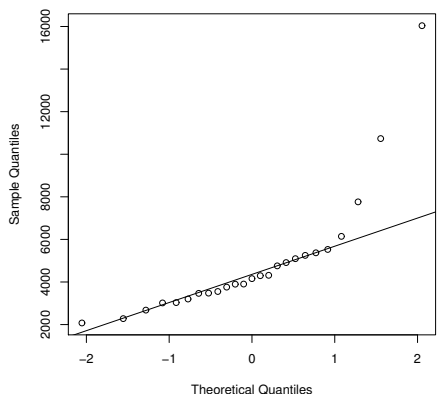

(o) $\mathrm{rCBV}$ (T2wLES, MS)

Figure 2.2: Normal plots of average rCBV values for each ROI and the respective patient groups.

Fischl and Dale 2000). FreeSurfer is referenced here as requested in the documentation (FreeSurfer 2016). FreeSurfer results were manually checked and corrected where necessary for every step as recommended by the FreeSurfer user guide.

Binary masks for the region of interest comprising the cortical grey matter (GMCort), the region of interest comprising cerebral white matter (WMROI), the region of interest comprising the thalamus (THAL) and the region of interest comprising the putamen (PUT) were extracted from the FreeSurfer output volume and converted to NIfTI file format using FMRIB's FSL software library (Jenkinson et al. 2012). T2w lesions were subsequently excluded from these ROI masks by inverting the respective T2w lesion mask and applying it to each ROI using FMRIB's FSL software library (Smith 2002). A brain-extracted version of the MPRAGE data was created using BET from FMRIB's FSL software library (Smith 2002). The first time-point of the original perfusion data was registered linearly to the brain-extracted MPRAGE data using 12 degrees 


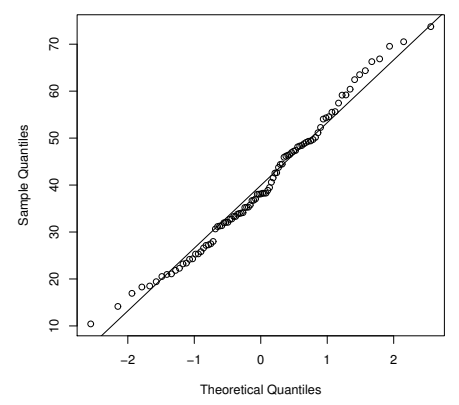

(a) $\mathrm{rCBF}$ (WMROI)

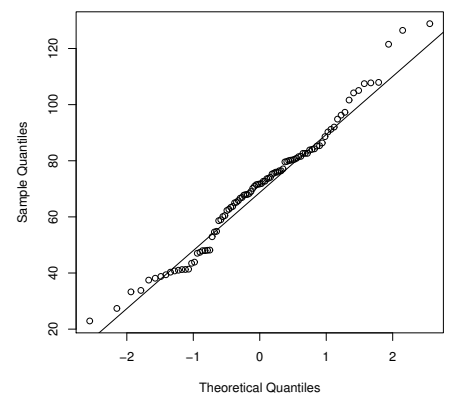

(d) $\mathrm{rCBF}$ (GMCort)

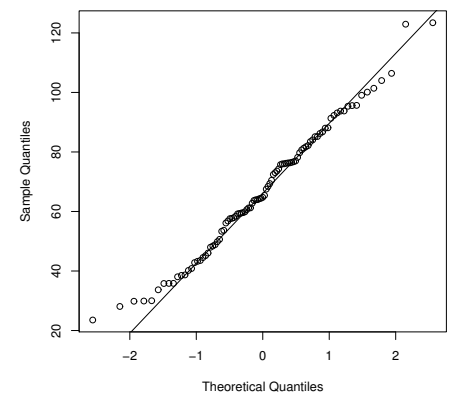

(g) $\mathrm{rCBF}(\mathrm{THAL})$

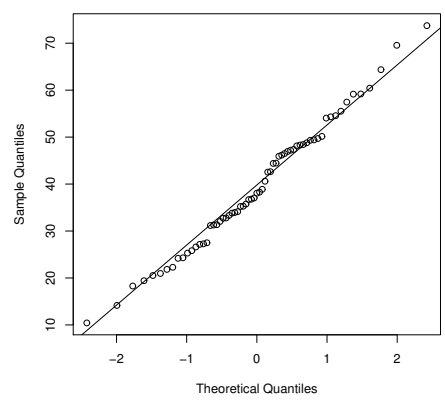

(b) $\mathrm{rCBF}$ (WMROI, CIS)

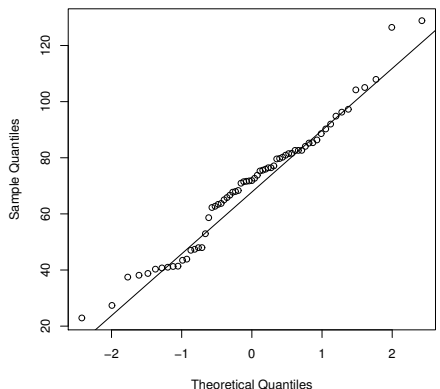

(e) rCBF (GMCort, CIS)

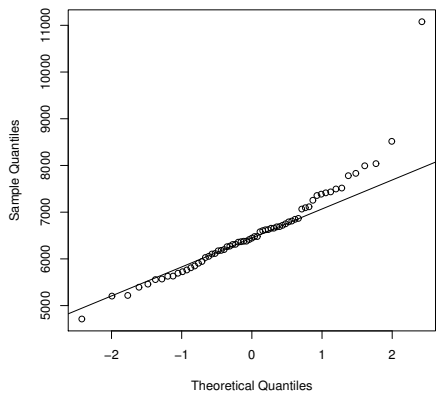

(h) rCBF (THAL, CIS)

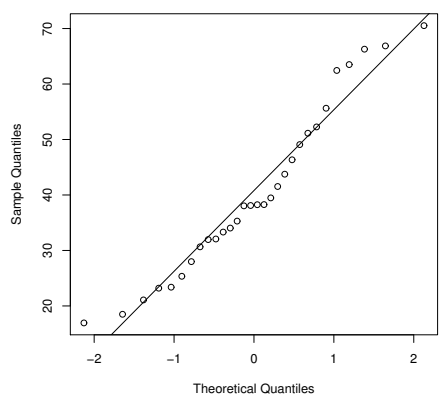

(c) rCBF (WMROI, MS)

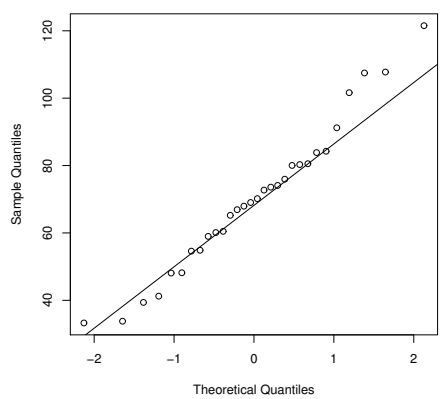

(f) $\mathrm{rCBF}$ (GMCort, MS)

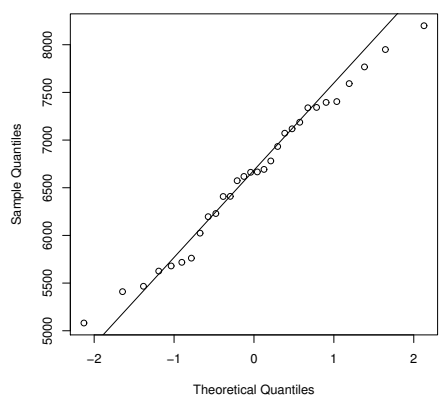

(i) $\mathrm{rCBF}$ (THAL, MS)

Figure 2.3: Normal plots of average rCBF values for each ROI and the respective patient groups (all, CIS, MS).

of freedom. The resulting transformation matrix was then inverted and applied to the MPRAGE data, thus effectively registering the MPRAGE data to the baseline perfusion image. All ROI masks and the lesion mask were also registered to perfusion resolution using this inverted transformation.

This registration comprises a change in resolution from the original MPRAGE space 


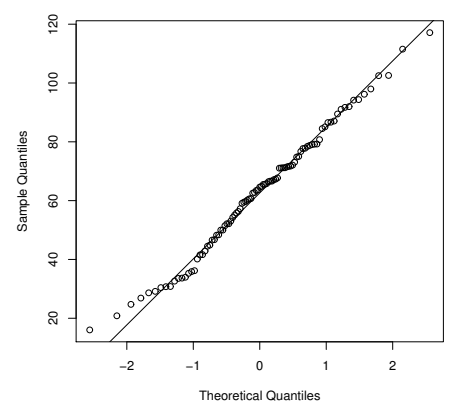

(j) $\mathrm{rCBF}(\mathrm{PUT})$

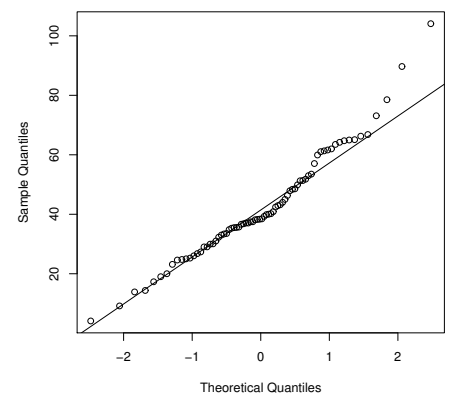

(m) $\mathrm{rCBF}(\mathrm{T} 2 \mathrm{wLES})$

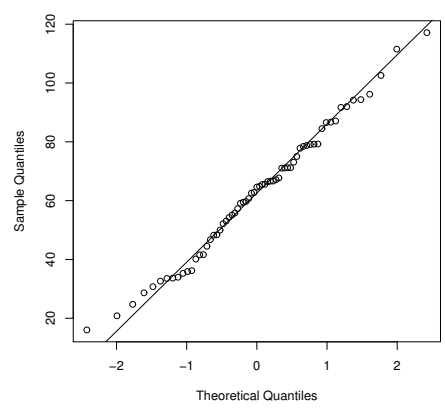

(k) $\mathrm{rCBF}(\mathrm{PUT}, \mathrm{CIS})$

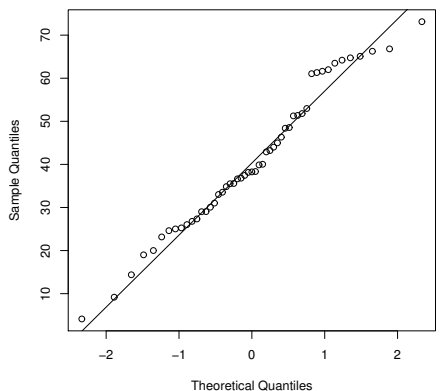

(n) $\mathrm{rCBF}$ (T2wLES, CIS)

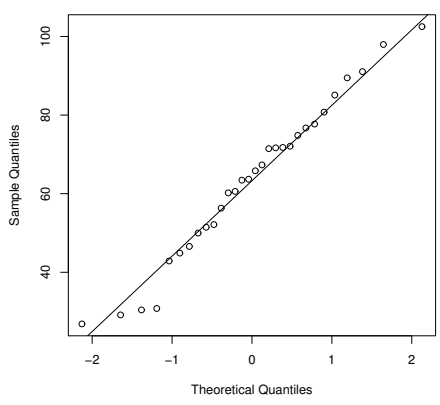

(l) $\mathrm{rCBF}(\mathrm{PUT}, \mathrm{MS})$

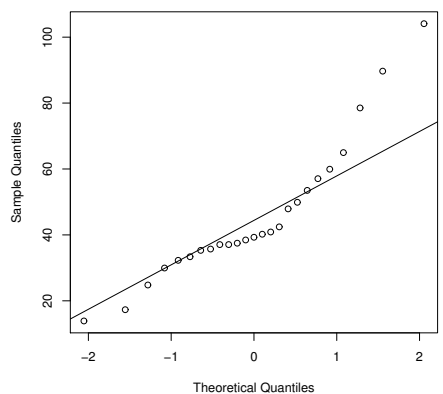

(o) $\mathrm{rCBF}(\mathrm{T} 2 \mathrm{wLES}, \mathrm{MS})$

Figure 2.3: Normal plots of average rCBF values for each ROI and the respective patient groups.

$\left(1 \times 1 \times 1 \mathrm{~mm}^{3}\right)$ to the resolution of the perfusion data $\left(1.8 \times 1.8 \times 5 \mathrm{~mm}^{3}\right)$. In the course of this downsampling, new voxels integrate information from several original voxels, which creates significant partial volume effects. To account for this change in resolution, a threshold was applied to all ROI masks. For all grey matter-derived ROI masks (GMCort, THAL, PUT) only those new voxels were accepted, of which at least a fraction of $80 \%$ was constructed from voxels originally belonging to the respective ROI. This aims to minimise partial volume effects while still keeping most of the original form and size of the ROI. For region of interest comprising the hyperintense lesions on T2-weighted magnetic resonance images (T2wLES) only new voxels were accepted, of which at least a fraction of $70 \%$ was constructed from original $\mathrm{T} 2 \mathrm{w}$ lesion voxels. A slightly larger partial volume effect was accepted for $\mathrm{T} 2 \mathrm{w}$ lesions in favour of more lesions surviving the downsampling. This is effectively in order to make sure the ROIs only contain normal appearing matter. Still 8 lesions masks were levelled by the registration. 
ROI analysis was carried out using FMRIB's FSL software library (Jenkinson et al. 2012). All respective ROI masks (GMCort, WMROI, PUT, THAL, T2wLES) were applied to all respective perfusion masks (rCBF, $\mathrm{rCBV}$ ) and calculated the mean of all non-zero voxels.

\subsection{Statistics}

Subjects were dichotomised according to their diagnosis in a subgroup of subjects diagnosed with clinically isolated syndrome (gCIS) and a subgroup of subjects diagnosed with relapsing-remitting multiple sclerosis (gMS). All statistical work was carried out using version 2.15.3 of $\mathrm{R}$ (http://www.R-project.org). Since all derived perfusion parameters are not normally distributed (cf. Figures 2.3 and 2.2), non-parametric methods were used: median and interquartile range (IQR) to describe the data, two-sided MannWhitney-U-Tests (MWU-Tests) to compare the distribution of different groups. IQRs were calculated as recommended by Hyndman and Fan (1996). A multiple regression model was used to evaluate relations between the various parameters and possible interactions simultaneously. Results were corrected for multiple testing where appropriate using the Bonferroni-Holm-correction (Holm 1979). 


\section{Results}

The two subject subgroups (gCIS and gMS) are similar with respect to demographic characteristics, though not in size, cf. Table 3.1. gCIS is twice the size of gMS, but the gender ratios are similar for both groups. The gender ratios of approximately $F: M=2: 1$ are also characteristic of a population of MS patients. There is no significant difference between the two groups with respect to age, EDSS and brain volumes (compare Table 3.1). There is a significant difference between both groups with respect to disease duration (DisDur), compare Table 3.1 .

\section{$3.1 \quad$ Volumes}

An overview of derived ROI volumes can be found in Table 3.2. Median and IQR are similar for gCIS and gMS. The results of the MWU-Tests implicate that both samples, gCIS and gMS, originate from the same statistical population for each respective ROIs. In particular, this comprises that there is no significant difference between the medians of both subject subgroups for each ROI.

\subsection{Perfusion parameters}

An overview of derived perfusion parameters can be found in Table 3.3, Table 3.4, Figure 3.1. Figure 3.2 and Figure 3.3. Median and IQR are similar for both groups in each ROI for both, rCBV and rCBF, respectively, cf. Figure 3.4. Figure 3.5, Table 3.3 and Table 3.4 


\begin{tabular}{|c|c|c|c|}
\hline & gCIS & gMS & $\begin{array}{l}\text { MWU-Test } \\
\text { (p-values) }\end{array}$ \\
\hline Subjects & 65 & 30 & \\
\hline Gender & Male: $21(32 \%)$ & Male: $8(27 \%)$ & \\
\hline & Female: $44(68 \%)$ & Female: $22(73 \%)$ & \\
\hline Age (years) & $\begin{array}{c}33 \pm 8 \\
(20-56)\end{array}$ & $\begin{array}{c}32 \pm 9 \\
(18-52)\end{array}$ & 0.39 \\
\hline EDSS & $\begin{array}{c}1.5 \\
(0-3.5)\end{array}$ & $\begin{array}{c}1.5 \\
(0-4)\end{array}$ & 0.18 \\
\hline $\begin{array}{l}\text { Disease duration } \\
\text { (months) }\end{array}$ & $\begin{array}{c}4 \\
(1-36)\end{array}$ & $\begin{array}{c}7.5 \\
(10-38)\end{array}$ & $1.60 \times 10^{-3}$ \\
\hline $\operatorname{NBV}\left(\mathrm{cm}^{3}\right)$ & $\begin{array}{c}1711 \pm 93 \\
(1451-1973)\end{array}$ & $\begin{array}{c}1698 \pm 86 \\
(1588-1915)\end{array}$ & 0.26 \\
\hline $\operatorname{NGMV}\left(\mathrm{cm}^{3}\right)$ & $\begin{array}{c}694 \pm 40 \\
(590-804)\end{array}$ & $\begin{array}{c}689 \pm 35 \\
(624-757)\end{array}$ & 0.52 \\
\hline $\operatorname{NWMV}\left(\mathrm{cm}^{3}\right)$ & $\begin{array}{c}1018 \pm 59 \\
(862-1168)\end{array}$ & $\begin{array}{c}1009 \pm 56 \\
(919-1158)\end{array}$ & 0.27 \\
\hline
\end{tabular}

Table 3.1: Comparison of the two different groups with respect to demographics. Median given for EDSS and disease duration, mean \pm standard deviation given for all other numeric parameters. Ranges give minimal and maximal values. Significant p-values are given in bold print.

\begin{tabular}{c|cccc}
\hline & $\begin{array}{c}\text { All } \\
(n=95)\end{array}$ & $\begin{array}{c}\text { CIS } \\
(n=65)\end{array}$ & $\begin{array}{c}\text { MS } \\
(n=30)\end{array}$ & $\begin{array}{c}\text { MWU-Test } \\
(\mathrm{p} \text {-values })\end{array}$ \\
\hline Cortex & M: 465 & M: 465 & M: 463 & 0.9 \\
$\left(\mathrm{~cm}^{3}\right)$ & IQR: 64 & IQR: 76 & IQR: 50 & \\
THAL & M: 15 & M: 15 & M: 14 & 0.6 \\
$\left(\mathrm{~cm}^{3}\right)$ & IQR: 3 & IQR: 3 & IQR: 2 & \\
PUT & M: 11 & M: 11 & M: 11 & 0.6 \\
$\left(\mathrm{~cm}^{3}\right)$ & IQR: 2 & IQR: 2 & IQR: 2 & \\
\hline
\end{tabular}

Table 3.2: Descriptive statistics for the different FreeSurfer-derived volumes; M: median. 


\begin{tabular}{c|cccc}
\hline & All & CIS & MS & MWU-Test \\
& $(n=95)$ & $(n=65)$ & $(n=30)$ & $($ p-values $)$ \\
\hline GMCort & M: 7548 & M: 7531 & M: 7566 & 0.99 \\
& IQR: 617 & IQR: 640 & IQR: 500 & \\
THAL & M: 6574 & M: 6444 & M: 6664 & 0.43 \\
& IQR: 1087 & IQR: 932 & IQR: 1337 & \\
PUT & M: 7595 & M: 7515 & M: 7666 & 0.15 \\
& IQR: 1000 & IQR: 1129 & IQR: 662 & \\
WMROI & M: 3957 & M: 3797 & M: 4095 & 0.72 \\
& IQR: 1123 & IQR: 1098 & IQR: 1206 & \\
T2wLES* & M: 3900 & M: 3782 & M: 4158 & 0.21 \\
& IQR: 1947 & IQR: 2092 & IQR: 1913 & \\
\hline
\end{tabular}

Table 3.3: Descriptive statistics for rCBV. ${ }^{*}$ Number of observations is different for T2wLES (gCIS: $n=51$, gMS: $n=25$, All: $n=76$ ), see Section 2.2.4 M: median.

\subsubsection{Group Comparisons}

There are several ways to look at group differences for the derived perfusion metrics and the results from the different comparisons are heterogeneous:

Comparing gCIS and gMS with respect to rCBV. The results of the MWUTests implicate that both samples, gCIS and gMS, originate from the same statistical population for each respective ROIs (compare Table 3.3). In particular, this comprises that there is no significant difference between the medians of each subject group for the respective ROI.

Comparing gCIS and gMS with respect to $\mathrm{rCBF}$. The results of the MWUTests implicate that both samples, gCIS and gMS, originate from the same statistical population for each respective ROIs (compare Table 3.4). In particular, this comprises that there is no significant difference between the medians of each subject subgroup for the respective ROI.

Comparing tuples of ROI within gCIS with respect to rCBV. For every tuple that consists of one grey matter ROI (GMCort, THAL, PUT) and one white matter ROI (WMROI, T2wLES) each, the results of the MWU-Tests implicate that rCBV of the two ROI do not originate from the same statistical population. In particular, this implicates that the medians of both respective ROIs also differ (see superdiagonal half of Table 3.5). Additionally, all possible tuples of grey matter ROIs differ in distribution and median, 


\begin{tabular}{c|cccc}
\hline & $\begin{array}{c}\text { All } \\
(n=95)\end{array}$ & $\begin{array}{c}\text { CIS } \\
(n=65)\end{array}$ & $\begin{array}{c}\text { MS } \\
(n=30)\end{array}$ & $\begin{array}{c}\text { MWU-Test } \\
(\mathrm{p} \text {-values })\end{array}$ \\
\hline GMCort & M: 72 & M: 72 & M: 70 & 0.80 \\
& IQR: 28 & IQR: 31 & IQR: 26 & \\
THAL & M: 65 & M: 65 & M: 65 & 0.94 \\
& IQR: 32 & IQR: 32 & IQR: 35 & \\
PUT & M: 65 & M: 65 & M: 65 & 0.78 \\
& IQR: 31 & IQR: 33 & IQR: 27 & \\
WMROI & M: 38 & M: 38 & M: 38 & 0.86 \\
& IQR: 18 & IQR: 19 & IQR: 21 & \\
T2wLES* & M: 38 & M: 38 & M: 39 & 0.49 \\
& IQR: 22 & IQR: 23 & IQR: 20 & \\
\hline
\end{tabular}

Table 3.4: Descriptive statistics for rCBF. ${ }^{*}$ Number of observations is different for T2wLES (CIS: $n=51$, MS: $n=25$, All: $n=76$ ), see Section 2.2.4: M: median.

except for the tuple consisting of (GMCort,PUT) (see superdiagonal half of Table 3.5). Conversely, the results of the MWU-Test indicate that two sets of rCBV values do not differ in distribution or median for WMROI and T2wLES (compare superdiagonal half of Table 3.5.

Comparing tuples of ROI within gCIS with respect to rCBF. For every tuple that consists of one grey matter ROI (GMCort, THAL, PUT) and one white matter ROI (WMROI, T2wLES) each, the results of the MWU-Tests implicate that rCBV of the two ROI do not originate from the same statistical population. In particular, this implicates that the medians of both respective ROIs also differ (see superdiagonal half of Table 3.6). Additionally, all possible tuples of grey matter ROIs differ in distribution and median (see superdiagonal half of Table 3.6). Conversely, the results of the MWUTest indicate that two sets of rCBV values do not differ in distribution or median for WMROI and T2wLES (compare superdiagonal half of Table 3.6).

Comparing tuples of ROI within gMS with respect to rCBV. For every tuple that consists of one grey matter ROI (GMCort, THAL, PUT) and one white matter ROI (WMROI, T2wLES) each, the results of the MWU-Tests implicate that rCBV of the two ROI do not originate from the same statistical population. In particular, this implicates that the medians of both respective ROIs also differ (see subdiagonal half of Table 3.5). Additionally, all possible tuples of grey matter ROIs differ in distribution and median, 
except for the tuple consisting of (GMCort,PUT) (see subdiagonal half of Table 3.5). Conversely, the results of the MWU-Test indicate that two sets of rCBV values do not differ in distribution or median for WMROI and T2wLES (compare subdiagonal half of Table 3.5.).

Comparing tuples of ROI within gMS with respect to rCBF. For every tuple that consists of one grey matter ROI (GMCort, THAL, PUT) and one white matter ROI (WMROI, T2wLES) each, the results of the MWU-Tests implicate that rCBV of the two ROI do not originate from the same statistical population. In particular, this implicates that the medians of both respective ROIs also differ (see superdiagonal half of Table 3.6.). Additionally, all possible tuples of grey matter ROIs differ in distribution and median, except for the two tuples consisting of (THAL,GMCort) and (THAL,PUT) (see superdiagonal half of Table 3.6). Conversely, the results of the MWU-Test indicate that two sets of rCBV values do not differ in distribution or median for WMROI and T2wLES (compare superdiagonal half of Table 3.6).

\subsubsection{Correlations}

Correlations were investigated from several angles and always in relation to NBV and EDSS, respectively.

For all data with respect to rCBV. A linear model containing mean rCBV for all ROI, age, gender and DisDur contributes significantly to explaining the systematic variation of NBV. Individually, mean rCBV for GMCort, THAL, WMROI, age, gender and DisDur are significant predictors for NBV, but mean rCBV for PUT is not. Conversely, a linear model containing mean rCBV for all ROI, age, gender and DisDur does not contribute significantly to explaining the systematic variation of EDSS (compare Table 3.7).

For all data with respect to rCBF. A linear model containing mean rCBF for all ROI, age, gender and DisDur contributes significantly to explaining the systematic variation of NBV. Individually, mean $\mathrm{rCBF}$ for THAL, WMROI, age and DisDur are significant predictors for NBV, but mean rCBF for GMCort, PUT and gender are not. Conversely, a linear model containing mean rCBF for all ROI, age, gender and DisDur does not contribute significantly to explaining the systematic variation of EDSS (compare Table 3.7). 
For gCIS with respect to $\mathrm{rCBV}$. A linear model containing mean $\mathrm{rCBV}$ for all ROI, age, gender and DisDur significantly contributes to explaining the systematic variation of NBV. Individually, mean rCBV for GMCort, THAL, WMROI and gender are significant predictors for NBV, but PUT, age and DisDur are not. Conversely, a linear model containing mean rCBV for all ROI, age, gender and DisDur does not contribute significantly to explaining the systematic variation of EDSS, see Table 3.8 . For gCIS with respect to rCBF. A linear model containing mean rCBF for all ROI, age, gender and DisDur significantly contributes to explaining the systematic variation of NBV. Individually, mean $\mathrm{rCBF}$ for THAL, WMROI and age are significant predictors for NBV, but GMCort, PUT, gender and DisDur are not. Conversely, a linear model containing mean rCBF for all ROI, age, gender and DisDur does not contribute significantly to explaining the systematic variation of EDSS (compare Table 3.8).

For gMS with respect to rCBV. For gMS a linear model containing mean rCBV for all ROI, age, gender and DisDur does not contribute significantly to explaining the systematic variation of either NBV or EDSS, see Table 3.9.

For gMS with respect to rCBF. For gMS a linear model containing mean rCBF for all ROI, age, gender and DisDur does not contribute significantly to explaining the systematic variation of either NBV or EDSS. More detailed statistical results can be found in Table 3.9 . 


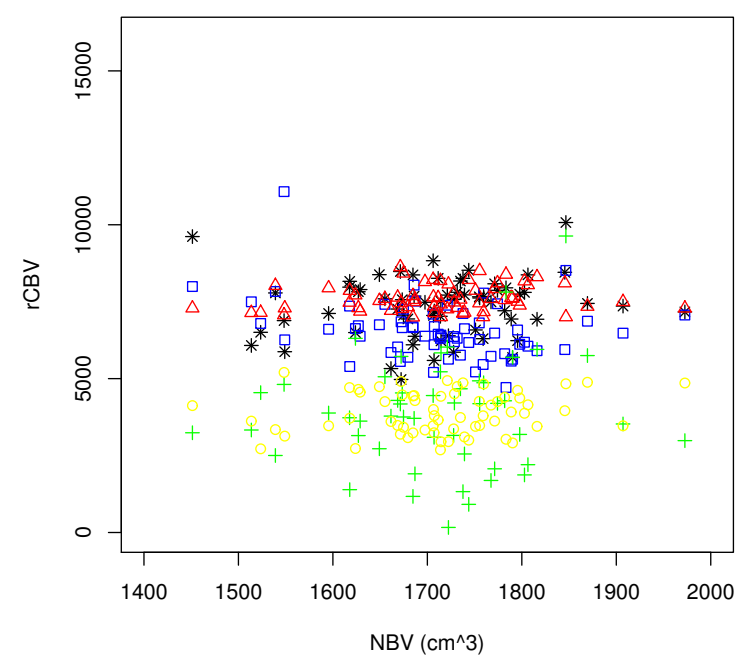

(a) gCIS

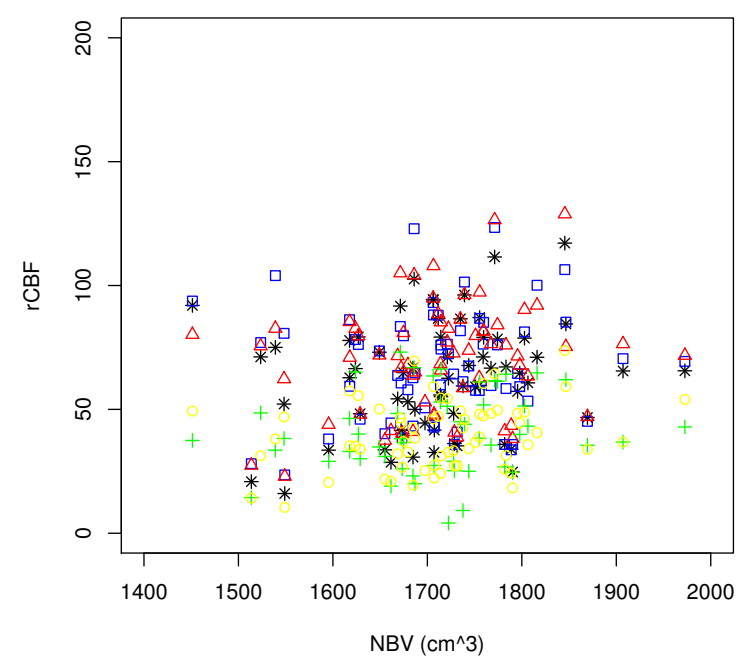

(c) gCIS

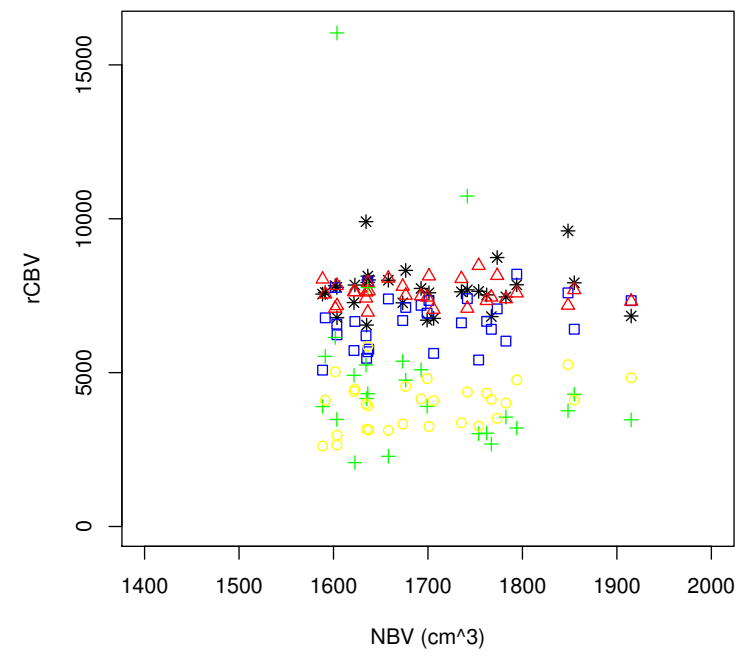

(b) gMS

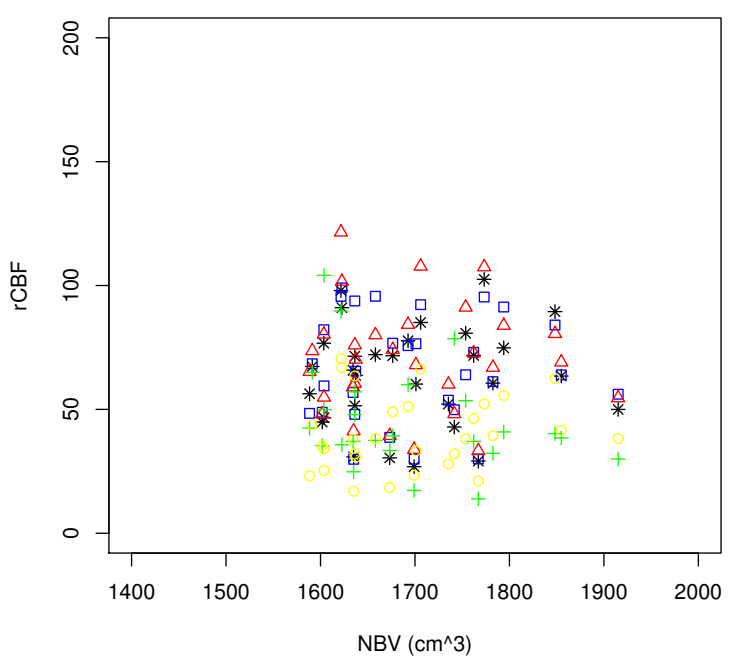

(d) gMS

Figure 3.1: Average $\operatorname{rCBV}((\mathrm{a}),(\mathrm{b}))$ and $\operatorname{rCBF}((\mathrm{c}),(\mathrm{d}))$ against NBV for all ROIs. GMCort: red triangles, THAL: blue squares, PUT: black asterisks, WMROI: yellow circles, T2wLES: green crosses. 


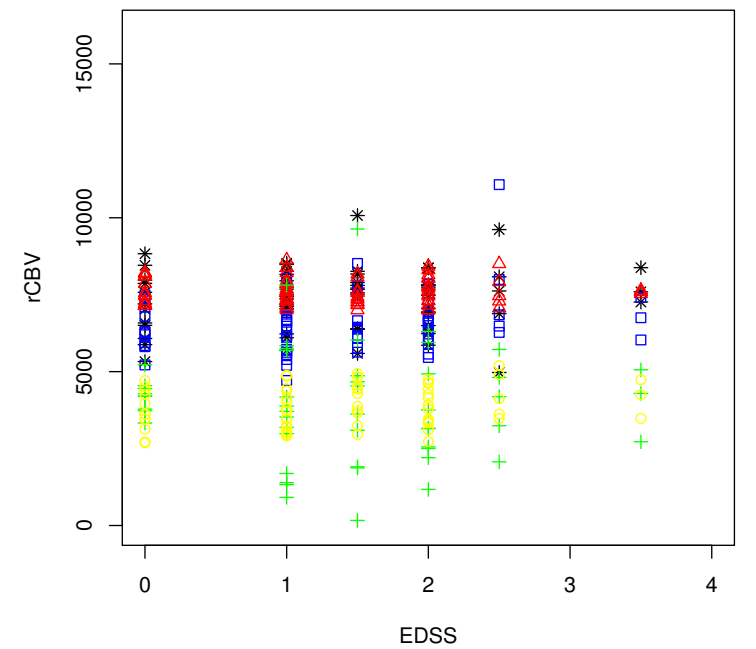

(a) gCIS

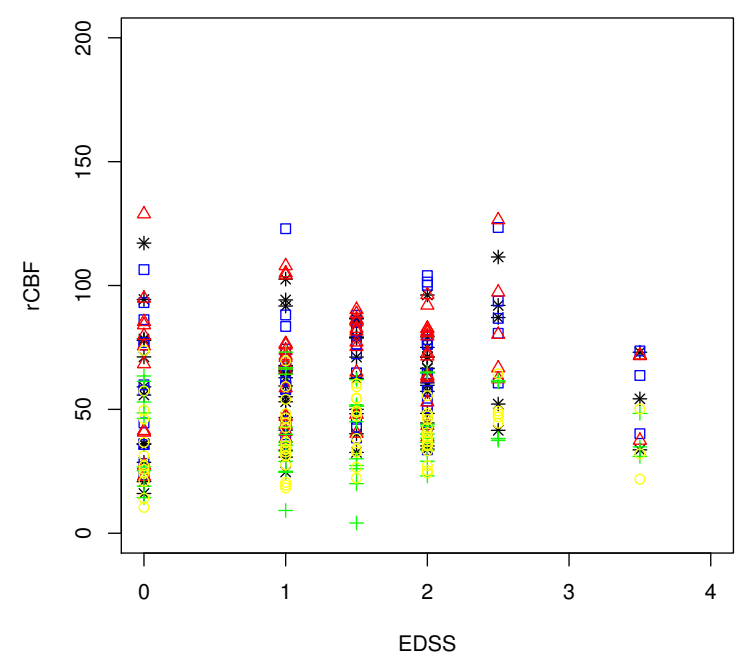

(c) gCIS

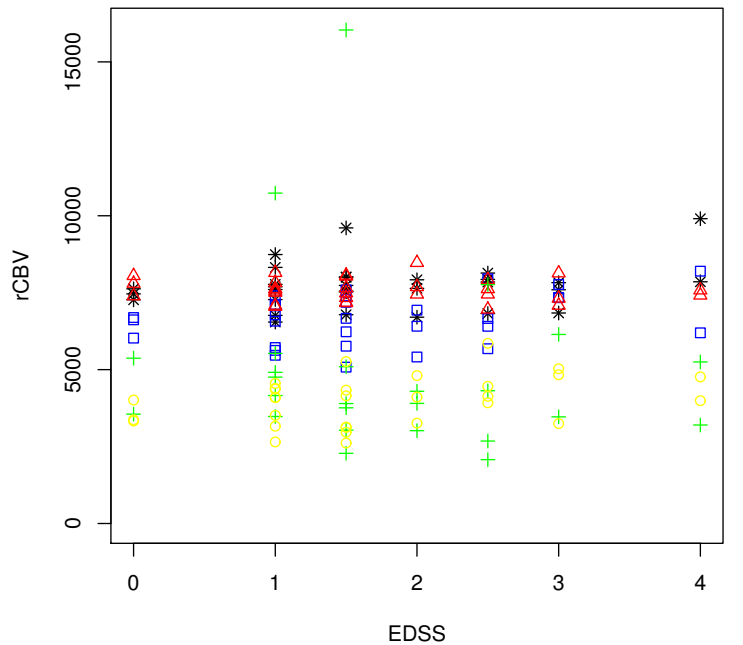

(b) gMS

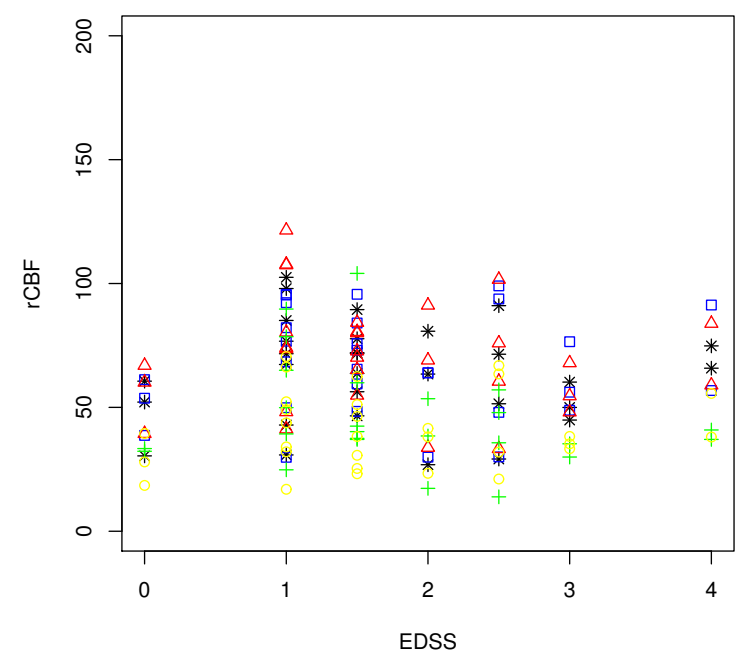

(d) gMS

Figure 3.2: Average $\operatorname{rCBV}((\mathrm{a}),(\mathrm{b}))$ and $\operatorname{rCBF}((\mathrm{c}),(\mathrm{d}))$ against EDSS for all ROIs. GMCort: red triangles, THAL: blue squares, PUT: black asterisks, WMROI: yellow circles, T2wLES: green crosses. 


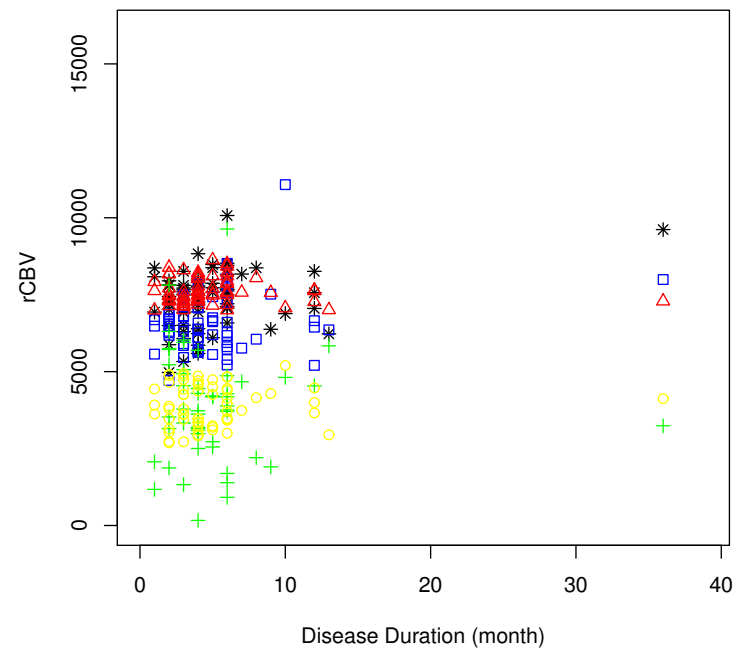

(a) gCIS

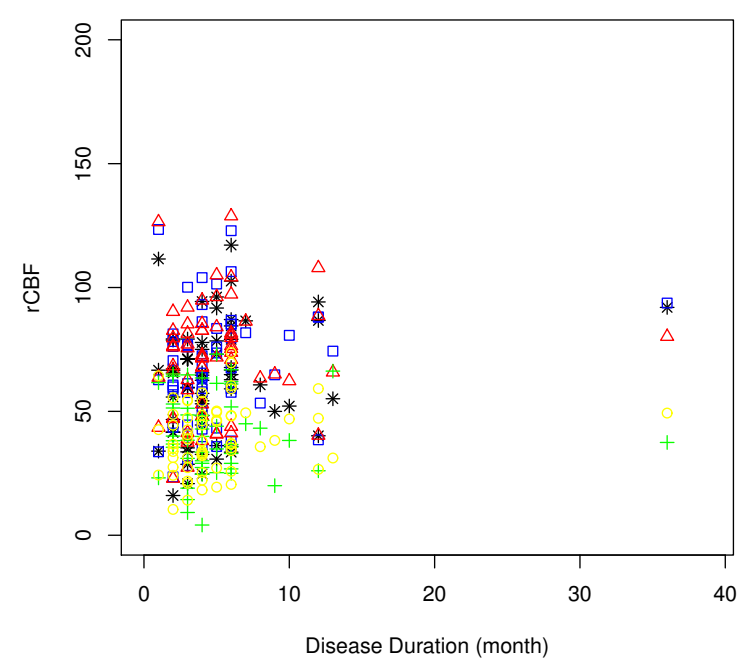

(c) gCIS

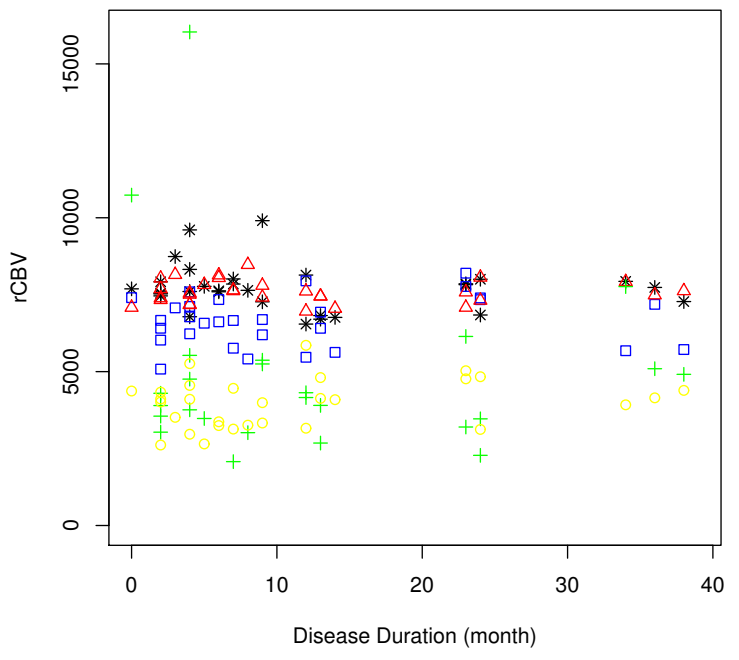

(b) gMS

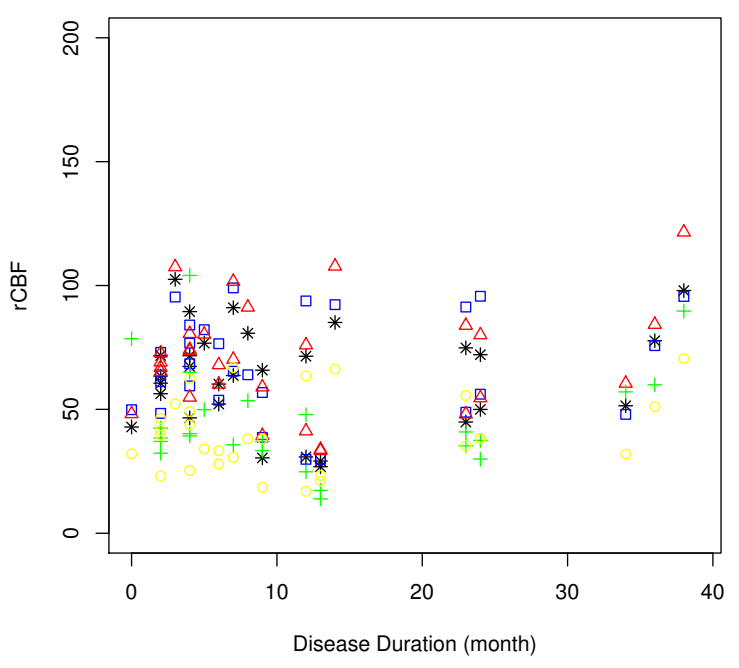

(d) gMS

Figure 3.3: Average $\operatorname{rCBV}((\mathrm{a}),(\mathrm{b}))$ and $\operatorname{rCBF}((\mathrm{c}),(\mathrm{d}))$ against disease duration for all ROIs. GMCort: red triangles, THAL: blue squares, PUT: black asterisks, WMROI: yellow circles, T2wLES: green crosses. 


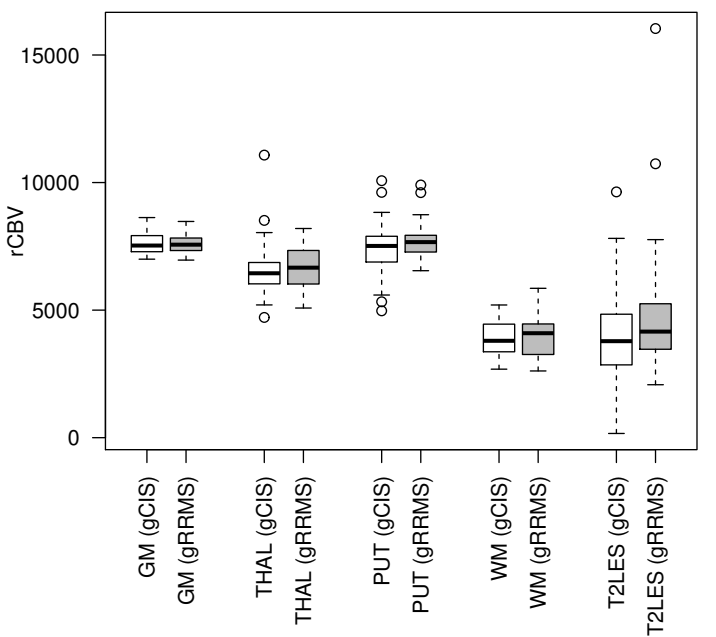

(a)

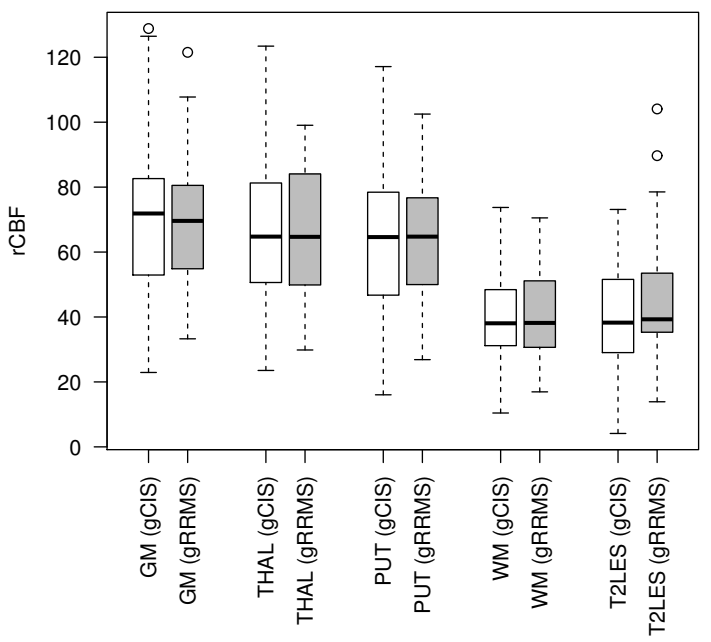

(b)

Figure 3.4: Boxplot for all ROIs and both patient groups (gCIS, gMS) for (a) rCBV and (b) rCBF.

\begin{tabular}{|c|c|c|c|c|c|}
\hline gMS gCIS & GMCort & THAL & PUT & WMROI & T2wLES \\
\hline GMCort & & $\begin{array}{c}1.69 \times \\
10^{-8}\end{array}$ & $\begin{array}{c}7.91 \times \\
10^{-2}\end{array}$ & $\begin{array}{l}1.72 \times \\
10^{-11}\end{array}$ & $\begin{array}{l}8.02 \times \\
10^{-17}\end{array}$ \\
\hline THAL & $\begin{array}{c}5.47 \times \\
10^{-5}\end{array}$ & & $\begin{array}{c}5.17 \times \\
10^{-6}\end{array}$ & $\begin{array}{c}1.72 \times \\
10^{-11}\end{array}$ & $\begin{array}{c}1.81 \times \\
10^{-14}\end{array}$ \\
\hline PUT & $\begin{array}{c}9.19 \times \\
10^{-1}\end{array}$ & $\begin{array}{c}1.93 \times \\
10^{-6}\end{array}$ & & $\begin{array}{c}1.72 \times \\
10^{-11}\end{array}$ & $\begin{array}{c}4.89 \times \\
10^{-16}\end{array}$ \\
\hline WMROI & $\begin{array}{c}1.86 \times \\
10^{-8}\end{array}$ & $\begin{array}{c}1.86 \times \\
10^{-8}\end{array}$ & $\begin{array}{c}1.86 \times \\
10^{-8}\end{array}$ & & $\begin{array}{c}8.85 \times \\
10^{-1}\end{array}$ \\
\hline T2wLES & $\begin{array}{c}4.47 \times \\
10^{-7}\end{array}$ & $\begin{array}{c}3.20 \times \\
10^{-6}\end{array}$ & $\begin{array}{c}3.10 \times \\
10^{-7}\end{array}$ & $\begin{array}{c}9.10 \times \\
10^{-1}\end{array}$ & \\
\hline
\end{tabular}

Table 3.5: Group comparisons with respect to rCBV. Table states corrected p-values of MWU-Tests for the tuple of ROIs defined by row/column. The superdiagonal half represents gCIS, the subdiagonal half represents gMS. Significant p-values are given in bold print. 


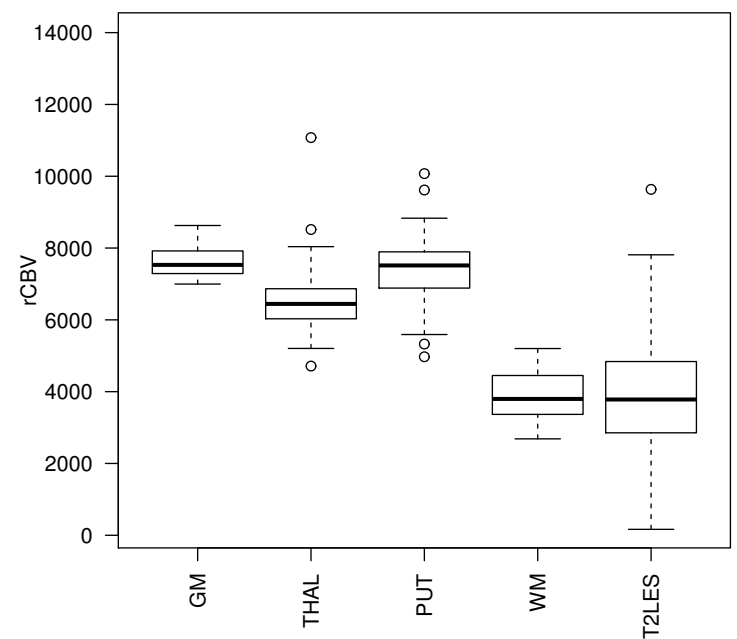

(a)

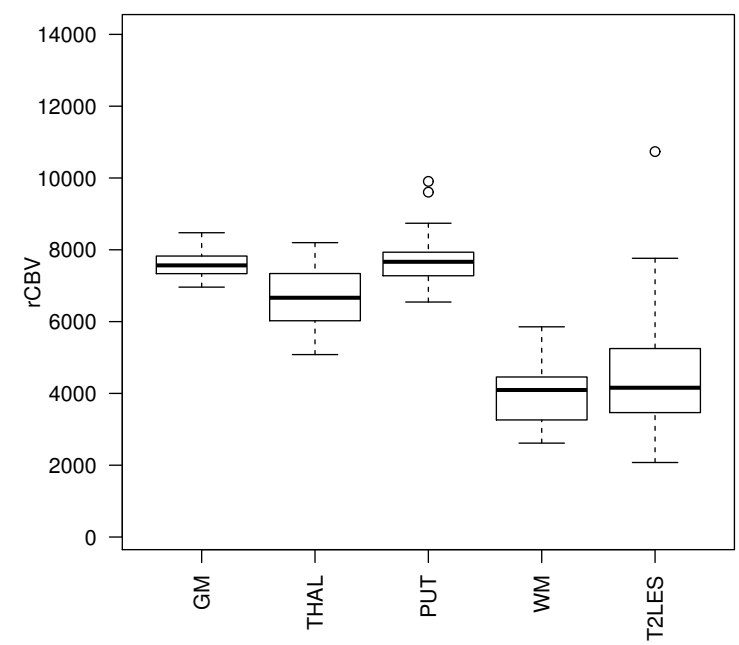

(c)

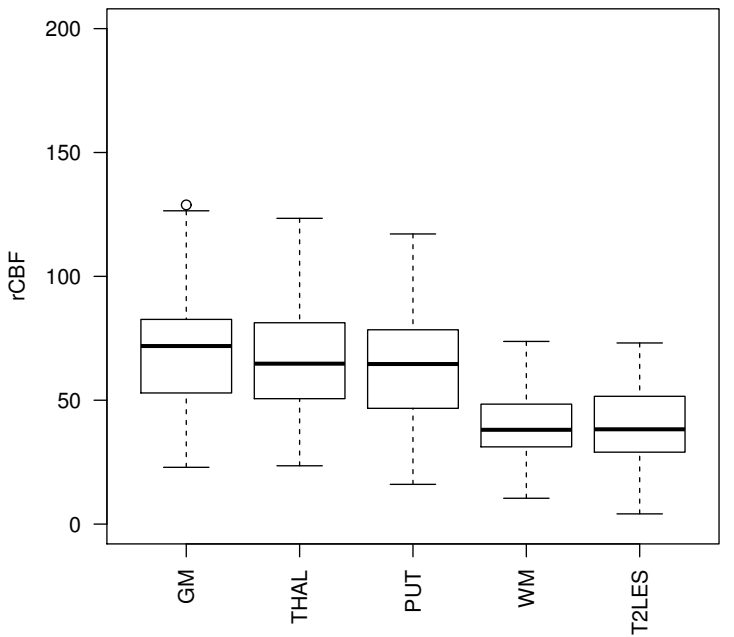

(b)

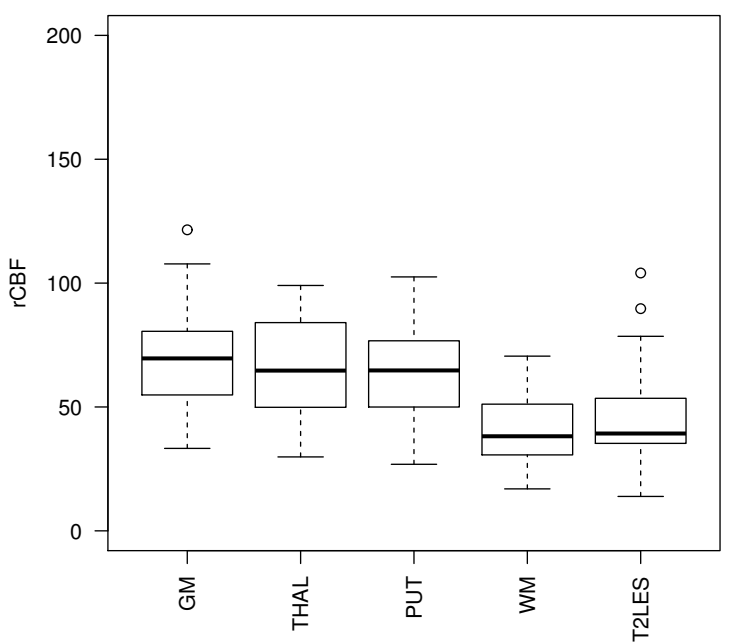

(d)

Figure 3.5: Boxplot for intragroup comparison for all ROIs and both, rCBV and rCBF, for gCIS ((a),(b)) and gMS ((c),(d)), respectively. 


\begin{tabular}{|c|c|c|c|c|c|}
\hline${ }_{\mathrm{gMS}} \mathrm{gCIS}$ & GMCort & THAL & PUT & WMROI & T2wLES \\
\hline GMCort & & $\begin{array}{c}1.60 \times \\
10^{-3}\end{array}$ & $\begin{array}{c}2.96 \times \\
10^{-9}\end{array}$ & $\begin{array}{c}2.46 \times \\
10^{-11}\end{array}$ & $\begin{array}{l}1.28 \times \\
10^{-10}\end{array}$ \\
\hline THAL & $\begin{array}{c}1.91 \times \\
10^{-1}\end{array}$ & & $\begin{array}{c}8.39 \times \\
10^{-4}\end{array}$ & $\begin{array}{c}2.46 \times \\
10^{-11}\end{array}$ & $\begin{array}{c}1.24 \times \\
10^{-8}\end{array}$ \\
\hline PUT & $\begin{array}{c}7.43 \times \\
10^{-5}\end{array}$ & $\begin{array}{c}1.91 \times \\
10^{-1}\end{array}$ & & $\begin{array}{c}2.46 \times \\
10^{-11}\end{array}$ & $\begin{array}{c}1.52 \times \\
10^{-6}\end{array}$ \\
\hline WMROI & $\begin{array}{c}1.86 \times \\
10^{-8}\end{array}$ & $\begin{array}{c}1.86 \times \\
10^{-8}\end{array}$ & $\begin{array}{c}1.86 \times \\
10^{-8}\end{array}$ & & $\begin{array}{c}6.60 \times \\
10^{-1}\end{array}$ \\
\hline T2wLES & $\begin{array}{c}2.34 \times \\
10^{-4}\end{array}$ & $\begin{array}{c}2.50 \times \\
10^{-3}\end{array}$ & $\begin{array}{c}6.60 \times \\
10^{-3}\end{array}$ & $\begin{array}{c}4.35 \times \\
10^{-1}\end{array}$ & \\
\hline
\end{tabular}

Table 3.6: Group comparisons with respect to rCBF. Table states corrected p-values of MWU-Tests for the tuple of ROIs defined by row/column. The superdiagonal half represents gCIS, the subdiagonal half represents gMS. Significant p-values are given in bold print.

\begin{tabular}{|c|c|c|c|c|}
\hline Metric: & \multicolumn{2}{|c|}{$\mathrm{rCBV}$} & \multicolumn{2}{|c|}{$\mathrm{rCBF}$} \\
\hline Target: & NBV & EDSS & NBV & EDSS \\
\hline F-Test & $6.34 \times 10^{-6}$ & $5.67 \times 10^{-2}$ & $2.89 \times 10^{-4}$ & $5.99 \times 10^{-2}$ \\
\hline $\operatorname{adj} . R^{2}$ & $2.80 \times 10^{-1}$ & $7.30 \times 10^{-2}$ & $2.04 \times 10^{-1}$ & $7.14 \times 10^{-2}$ \\
\hline GMCort & $5.63 \times 10^{-3}$ & $4.43 \times 10^{-1}$ & $5.11 \times 10^{-1}$ & $1.75 \times 10^{-1}$ \\
\hline THAL & $3.50 \times 10^{-2}$ & $5.15 \times 10^{-1}$ & $2.418 \times 10^{-2}$ & $6.31 \times 10^{-2}$ \\
\hline PUT & $7.75 \times 10^{-1}$ & $6.05 \times 10^{-1}$ & $9.83 \times 10^{-1}$ & $6.45 \times 10^{-1}$ \\
\hline WMROI & $1.09 \times 10^{-5}$ & $1.22 \times 10^{-1}$ & $3.09 \times 10^{-3}$ & $3.22 \times 10^{-1}$ \\
\hline Age & $8.73 \times 10^{-3}$ & $4.47 \times 10^{-1}$ & $8.39 \times 10^{-3}$ & $4.26 \times 10^{-1}$ \\
\hline Gender & $1.49 \times 10^{-2}$ & $8.41 \times 10^{-1}$ & $2.47 \times 10^{-1}$ & $7.32 \times 10^{-1}$ \\
\hline DisDur & $3.95 \times 10^{-2}$ & $9.26 \times 10^{-2}$ & $4.40 \times 10^{-2}$ & $5.40 \times 10^{-2}$ \\
\hline Intercept & $7.09 \times 10^{-07}$ & $2.75 \times 10^{-1}$ & $2.00 \times 10^{-16}$ & $1.22 \times 10^{-1}$ \\
\hline
\end{tabular}

Table 3.7: Multiple regression results for pooled data. Table states p-values of the respective t-test for each ROI. Significant p-values are given in bold print. 


\begin{tabular}{|c|c|c|c|c|}
\hline Metric: & \multicolumn{2}{|c|}{$\mathrm{rCBV}$} & \multicolumn{2}{|c|}{$\mathrm{rCBF}$} \\
\hline Target: & NBV & EDSS & NBV & EDSS \\
\hline F-Test & $5.25 \times 10^{-5}$ & $5.33 \times 10^{-1}$ & $3.65 \times 10^{-4}$ & $3.37 \times 10^{-1}$ \\
\hline $\operatorname{adj} . R^{2}$ & $3.38 \times 10^{-1}$ & 0 & $2.85 \times 10^{-1}$ & $1.77 \times 10^{-2}$ \\
\hline GMCort & $4.16 \times 10^{-2}$ & $4.16 \times 10^{-1}$ & $2.29 \times 10^{-1}$ & $8.87 \times 10^{-1}$ \\
\hline THAL & $1.26 \times 10^{-2}$ & $9.12 \times 10^{-1}$ & $1.61 \times 10^{-2}$ & $1.27 \times 10^{-1}$ \\
\hline PUT & $3.14 \times 10^{-1}$ & $9.83 \times 10^{-1}$ & $9.51 \times 10^{-1}$ & $2.63 \times 10^{-1}$ \\
\hline WMROI & $7.01 \times 10^{-4}$ & $2.68 \times 10^{-1}$ & $1.34 \times 10^{-2}$ & $4.77 \times 10^{-1}$ \\
\hline Age & $5.88 \times 10^{-2}$ & $3.01 \times 10^{-1}$ & $3.73 \times 10^{-2}$ & $2.18 \times 10^{-1}$ \\
\hline Gender & $1.82 \times 10^{-2}$ & $3.86 \times 10^{-1}$ & $3.34 \times 10^{-1}$ & $9.77 \times 10^{-1}$ \\
\hline DisDur & $7.96 \times 10^{-2}$ & $7.09 \times 10^{-1}$ & $7.42 \times 10^{-2}$ & $8.13 \times 10^{-1}$ \\
\hline Intercept & $3.91 \times 10^{-6}$ & $4.16 \times 10^{-1}$ & $1.10 \times 10^{-37}$ & $6.85 \times 10^{-1}$ \\
\hline
\end{tabular}

Table 3.8: Multiple regression results for gCIS. Table states p-values of the respective t-test for each ROI. Significant p-values are given in bold print.

\begin{tabular}{l|cc|cc}
\hline $\begin{array}{l}\text { Metric: } \\
\text { Target: }\end{array}$ & NBV & EDSS & NBV & EDSS \\
\hline F-Test & $2.38 \times 10^{-1}$ & $3.70 \times 10^{-1}$ & $4.64 \times 10^{-1}$ & $2.75 \times 10^{-1}$ \\
adj. R R $^{-1}$ & $9.69 \times 10^{-2}$ & $3.49 \times 10^{-2}$ & 0 & $1.77 \times 10^{-2}$ \\
GMCort & $2.19 \times 10^{-1}$ & $6.62 \times 10^{-1}$ & $1.74 \times 10^{-1}$ & $4.35 \times 10^{-2}$ \\
THAL & $5.08 \times 10^{-1}$ & $6.26 \times 10^{-1}$ & $6.26 \times 10^{-1}$ & $6.74 \times 10^{-1}$ \\
PUT & $6.15 \times 10^{-1}$ & $4.46 \times 10^{-1}$ & $3.30 \times 10^{-1}$ & $1.88 \times 10^{-1}$ \\
WMROI & $9.78 \times 10^{-2}$ & $4.07 \times 10^{-1}$ & $2.29 \times 10^{-1}$ & $5.33 \times 10^{-1}$ \\
Age & $2.64 \times 10^{-1}$ & $7.22 \times 10^{-1}$ & $3.45 \times 10^{-1}$ & $6.71 \times 10^{-1}$ \\
Gender & $3.60 \times 10^{-1}$ & $4.74 \times 10^{-1}$ & $2.09 \times 10^{-1}$ & $6.61 \times 10^{-1}$ \\
DisDur & $3.77 \times 10^{-1}$ & $2.87 \times 10^{-1}$ & $9.68 \times 10^{-1}$ & $8.12 \times 10^{-2}$ \\
Intercept & $6.64 \times 10^{-2}$ & $3.93 \times 10^{-1}$ & $7.23 \times 10^{-17}$ & $1.90 \times 10^{-1}$ \\
\hline
\end{tabular}

Table 3.9: Multiple regression results for gMS. Table states $\mathrm{p}$-values of the respective t-test for each ROI. Significant p-values are given in bold print. 


\section{Discussion}

Even though the two main groups (gCIS and gMS) are not comparable in size, they do not differ significantly in demographic characteristics, such as gender ratios and age, or measures of disease severity, such as EDSS and brain volumes. But there is a significant difference in disease duration. This can hardly be avoided, however, when comparing CIS, which is by definition a single incident, to RRMS, which comprises several incidents of which each would constitute a CIS. It is also possible that gCIS contains some subjects who really suffer from RRMS, but do not yet fulfil the dissemination criteria with respect to time (Polman et al. 2011). But since this study is part of a longitudinal setup, subjects were followed up for 24-48 months and diagnoses were updated where necessary. Thus the number of gCIS patients which really should be in gMS is likely comparatively small. Overall, the results presented in Chapter 3 are therefore likely to represent real effects rather than simply a biased group selection.

\subsection{Volumes}

There are no significant group differences for regional FreeSurfer-derived volumes (cf. Table 3.2). Loss of brain volume has been observed in early RRMS patients as well as in CIS patients (De Stefano et al. 2010), so generally this finding is consistent with the accepted understanding of the pathophysiology of MS. However, it should be noted that there is a small difference between gCIS and gMS with respect to disease duration. Therefore one might expect a small group difference with respect to regional volumes as well, simply because the underlying processes leading to neurodegeneration and brain volume loss have been going on for a longer period of time. But considering that FreeSurfer's sensitivity for correctly classifying a grey matter voxel was found to be only approximately $83-85 \%$ in ideal situations (Klauschen et al. 2009; Eggert et al. 
2012), such a small difference is likely to be obscured by FreeSurfer's sensitivity.

Moreover, the transformation of ROIs from the high-resolution MPRAGE image space into the space of the echo-planar perfusion image, which is also known to be geometrically distorted (Jezzard and Balaban 1995), presents another source of inaccuracy on two counts. Firstly, parts of several voxels are integrated into one new voxel, as the resolution significantly differs between the MPRAGE and the perfusion image. This causes a partial volume effect, which is accounted for by the threshold used in binarising the resulting mask (compare Section 2.2.4). The only way to be certain of not capturing any voxels outside of the original ROI would be to use a threshold of $100 \%$. However, because of the difference in resolution between the MPRAGE and the perfusion image, this would also exclude quite a number of voxels which largely consist of ROI tissue. Especially in non-uniform ROIs, this would influence the derived mean values. This problem was addressed by using a conservative threshold on all ROI masks during downsampling, in order to make sure that the registered ROI contains most of what was originally included in the ROI. Additionally, a more liberal threshold was chosen for T2wLES, in order to make sure that all other registered ROI masks do not contain lesional tissue.

Secondly, the echo-planar perfusion image is geometrically distorted Jezzard and Balaban 1995) in comparison with the MPRAGE, on which the ROI masks are initially defined. This is in part accounted for by using a linear transformation with 12 degrees of freedom to register the echo-planar perfusion image to the MPRAGE. But there are some problems with this method. The perfusion image displays rather insufficient grey/white contrast, so the alignment is based on other landmarks. So even with a good alignment of the brain outlines, there is not necessarily a good alinement's of the (deep) grey matter ROIs. Additionally, a linear transformation only allows for scaling but not for local distortion of the images via the registration, which does not completely capture the reality. The obvious solution to this problem would be to use a non-linear transformation for the registration process. However, there is probably not much to be gained by this approach. Using a non-linear transformation allows for local distortion, but there simply is not enough information in the echo-planar perfusion image (grey/white contrast, regional landmarks) to generate good local registration results with respect to the anatomical regions used here. Therefore using a non-linear transformation introduces a lot of uncontrollable distortion of the respective ROI, which is a likely to cause 
a loss in registration quality compared to a linear transformation as it is likely to cause an improvement in registration quality in this particular setting. So, in summary, even though the results of this study are consistent with what we know about MS pathology, these findings should not be interpreted as hard evidence that there is no difference in (regional) volumes between both groups.

\subsection{Perfusion parameters}

When interpreting results from perfusion studies, it should be kept in mind that the different processing methods might be accountable for some of the differences. DSC imaging only provides relative data and inherently depends on the application of the contrast agent bolus. Data is standardised and/or normalised according to different protocols, if at all. Especially in MS patients there can be substantial leaking of the constrast agent. Again there are several methods to correct for contrast agent leakage, but leakage correction is not always performed, making it even harder to assess the comparability of existing results. In this study, the leakage correction offered by the IB Neuro plug-in was used. There are no existing studies with respect to MS and the IB Neuro plug-in, but Boxerman et al. (2006) could show that leakage correction significantly improved the accuracy of rCBV estimation in gliomas with relevant contrast extravasation.

It should also be taken into account that as $\mathrm{rCBF}$ inherently depends on the manually defined AIF, which strongly depends on the vascular structure. An accurate acquisition of the AIF is essential for accurate results (van Osch et al. 2003). The AIF was defined by four manually chosen voxels in order to fully control this important process. However, it is possible that the four chosen voxels do not represent the arterial input sufficiently well, resulting in a bias in CBF. Generally, the arterial input will not be the same everywhere in the brain owing to circulation and vascular state as well as local vasoactivity (Mottet et al. 1997; Conturo et al. 2005). AIF signal-to-noise-ratio was optimised in this study by choosing a distant branch of the medial cerebral artery as suggested by Ebinger et al. (2010). But using AIF-derived parameters is still afflicted with some uncertainty. Additionally, a rater bias cannot be excluded. But a rater bias does not seem particularly likely, as there was only one rater and definition of the AIFs followed strict criteria (cf. Section 2.2.3. 


\subsubsection{Group Comparisons}

There is no significant group difference between gCIS and gMS with respect to both, rCBV and $\mathrm{rCBF}$, for each respective ROI, suggesting at first glance that there is no real difference in perfusion between both groups. The literature on perfusion changes in MS is heterogeneous, with respect to methods as well as with respect to results.

\section{Comparing subgroups with respect to cerebral blood volume (CBV)}

Peruzzo et al. (2012) found a significant reduction in CBV in cortical lesions compared with NAGM in RRMS patients. Adhya et al. (2006) found reduced CBV values in NAWM in RRMS patients compared to healthy controls. Papadaki et al. (2012) found comparable CBV values in RRMS patients and healthy controls. Inglese et al. (2007) also found no difference between RRMS patients and healthy controls with respect to CBV. However, Papadaki et al. (2012) found elevated CBV values in CIS patients compared to RRMS patients. But it should be kept in mind that subtle differences between the two subject groups might simply be obscured by the inherent methodological uncertainties. Overall, the results presented here most likely support the findings of Papadaki et al. (2012) and Inglese et al. (2007).

\section{Comparing subgroups with respect to $\mathrm{CBF}$}

Findings on $\mathrm{CBF}$ are more homogeneous,as most studies found a reduction in $\mathrm{CBF}$ when comparing patients to healthy controls. Two studies found a significant reduction in CBF for the nucleus caudatus and the thalamus in CIS patients compared to healthy controls (Papadaki et al. 2012; Papadaki et al. 2014b). However, there was no difference between CIS patients and healthy controls with respect to CBF values in the putamen and the NAWM (Papadaki et al. 2012). Using arterial spin labelling (ASL), Rashid et al. (2004) found reduced perfusion in CIS patients compared to healthy controls in both cortical and deep GM. Hojjat et al. (2016) found a significant reduction in CBF values in cognitively impaired RRMS patients compared to healthy controls in several cortical regions. Hojjat et al. (2016) also found a significant reduction in several deep grey matter regions including the thalamus and the putamen. Debernard et al. (2014) found reduced CBF values in RRMS patients compared to healthy controls in several cortical areas and deep grey matter as well. Papadaki et al. (2012) found siginifcantly decreased CBF 
values in all deep GM in RRMS patients compared to healthy controls. Inglese et al. (2007) also found CBF values in the thalamus, the putamen and the caudate nuclei to be reduced in RRMS patients compared with healthy controls. Conversely, Varga et al. (2009) only found a significant reduction in CBF in the putamen of RRMS patients in comparison to healthy controls, but not for the rest of the deep GM. Using ASL, Rashid et al. (2004) found reduced perfusion in RRMS patients compared to healthy controls in both cortical and deep GM. Ge et al. (2005b) also found reduced CBF in lesions and NAWM in RRMS patients compared with healthy controls. Papadaki et al. (2012) found siginifcantly decreased CBF values in NAWM in RRMS compared to healthy controls. Adhya et al. (2006) and Law et al. (2004) also found reduced CBF in NAWM in RRMS patients compared with healthy controls. But conversely, Rashid et al. (2004) also found elevated perfusion in cerebral white matter (WM) in RRMS patients compared to healthy controls using ASL. Varga et al. (2009) found RRMS patients to show significantly reduced CBF values in the putamen in comparison with CIS patients. Similarly, Papadaki et al. (2012) found RRMS patients to show significantly reduced CBF values in NAWM and deep GM compared to CIS patients, with the exception of the caudate nuclei. Papadaki et al. (2012) even found that there is no overlap in the distribution of CBV between CIS and RRMS patients, even after correcting for disease duration, EDSS score, T1-weighted lesion volume and T2w lesion volume.

In summary, all existing studies found reduced CBF values in MS patients, regardless of the subtype. The findings of this study neither argue for such a difference in CBF between patients and healthy controls, nor against it. If CBF was unaffected in both subgroups of subjects, there would be no difference. But equally, there would be no difference between both patient subgroups, if CBF was affected to a similar degree in both subgroups. Since additional to all the studies, which found a decrease in CBF in MS patients compared to healthy controls, there is considerable evidence that CIS patients already show signs of demyelination and diffuse axonal damage as well (Iannucci et al. 2000; Brex et al. 2001; Miller et al. 2005a), the most likely interpretation of the presented data is that the deficit in $\mathrm{rCBF}$ is similar in both subgroups. But in direct comparison several studies found a difference between CIS and RRMS patients, suggesting that CBF is more severely affected in RRMS. This is not reflected in the findings of this study. But it should be kept in mind that subtle differences between the two subject groups might simply be obscured by the inherent methodological uncertainties. Moreover, it 
should be noted that most of the results detailed above found differences in CBF but not in CBV. While this might simply reflect a real effect, it could equally well reflect the dependence of $\mathrm{CBF}$ on a somewhat arbitrarily defined AIF.

\section{Comparing ROIs within subject subgroups}

Even though there is no difference between the two subgroups for any ROI, there are differences between several pairs of ROIs within each group. Within both groups, there is a significant difference for each pair of a grey matter ROI (GMCort, THAL, PUT) and WMROI, respectively. This is expected considering the different structure of grey matter and white matter. There is a significant difference between PUT and THAL for $\mathrm{rCBV}$ in gCIS and gMS and for $\mathrm{rCBF}$ only in gCIS. That the difference in $\mathrm{rCBF}$ between THAL and PUT only exists for gCIS could suggest that rCBF is affected to the same degree in THAL and PUT in gMS, but not in gCIS. This finding is explicitly consistent with the results and the idea of Varga et al. (2009) that perfusion change is an ongoing process in MS, which reaches the putamen before it reaches the thalamus. Such a spreading perfusion change would result in exactly the pattern of differences in rCBF between THAL and PUT reported above. But Papadaki et al. (2012) found that both, the putamen and the thalamus displayed reduced CBF values in RRMS patients compared to CIS patients, which seems to be incompatible with the findings discussed above. However, it should be noted that these results could also be consistent with the presented findings, as a difference between THAL and PUT does not reflect whether ROI are affected, but whether they are affected to a different degree. The idea that rCBF is the first parameter to be affected, is in line with the findings of Law et al. (2004) and Varga et al. (2009), who found a difference in CBF between different groups of subjects, but not in CBV. Yet, since this study comprises only cross-sectional data and the gMS patients are not the ones who were in gCIS before, the evidence in support of a dynamically spreading change in perfusion remains circumstantial. And it should also be kept in mind that the results presented here are equally open to the interpretation that there is no difference between THAL and PUT in gMS, because both regions are unaffected in gMS, while one of them is affected in gCIS. 


\subsubsection{Correlations}

A multiple regression model comprising all ROIs with respect to either $\mathrm{rCBV}$ or $\mathrm{rCBF}$, as well as age, gender and disease duration was used to try to explain the variation in NBV and EDSS, respectively. NBV was chosen as the target variable because brain volume loss is considered to be a sensitive measure of neurodegeneration (Zivadinov and Bakshi 2004; Zivadinov et al. 2008; Barkhof et al. 2009). The extent of (early) brain volume loss has also been shown to be a predictor of more severe progression in terms of cognitive impairment and disability in MS patients (Bermel and Bakshi 2006; Simon 2006; Amato et al. 2007; Minneboo et al. 2008; Fisniku et al. 2009; Filippi and Rocca 2011; Zivadinov et al. 2013b; De Stefano et al. 2014; Jacobsen et al. 2014). EDSS was chosen as a comprehensive marker of clinical affection. The resulting adjusted $\mathrm{R}^{2}$ are comparatively bad, suggesting the model does not capture most of the systematic variation in NBV and EDSS, respectively. However, this is only mildly relevant, as the purpose is to simultaneously analyse correlations - and not to find the best predictors for NBV and EDSS, respectively.

On a note of caution, it should be noted that brain volume loss has been found in various studies to proceed at a rate of approximately $0.5-1.05 \%$ per year in MS patients and at a rate of approximately $0.1-0.3 \%$ per year in healthy individuals (Simon 2006; Fotenos et al. 2008; Fisher et al. 2008; Barkhof et al. 2009; De Stefano et al. 2010; De Stefano et al. 2014; Vollmer et al. 2015). However, SIENAX can reliably (Smith 2002; Sormani et al. 2004, Anderson et al. 2006; Smith et al. 2007) estimate brain volume with an accuracy of $0.5-1 \%$ (Smith et al. 2001; Smith 2002; De Stefano et al. 2007). As the enrolled CIS patients and most of the enrolled MS patients find themselves at the beginning of the course of the disease, the brain volume loss attributable to MS will probably be small. Therefore the brain volume loss due to the disease is expected to be only in the range of the accuracy of the method. Also, considering that likely only a fraction of the change in NBV which are attributable to the disease is caused by perfusion changes, the overall goodness of fit (as represented by adjusted $R^{2}$ ) is expected to be comparatively small even in ideal situations. This does not invalidate the approach as a means of simultaneously examining correlations.

Based on the group comparisons there is no a priori justification for excluding specific ROIs from the regression models. Therefore all ROIs were included. No model selection 
algorithms were applied and no further effort was made to optimise the amount of variability in NBV and EDSS explained by the respective model. Adhering to this argument, it would make most sense to include all perfusion parameters in the same model. However, this would likely render all perfusion-related predictors artificially insignificant, as rCBV and rCBF are inherently linearly correlated. Therefore rCBV and rCBF were investigated separately. Generally, it should be kept in mind that existing correlations between any two or more predictors would render all of the respective ttests insignificant. Also, an existing weak correlation could manifest itself only in $\mathrm{rCBF}$ and not in $\mathrm{rCBV}$, if it was amplified by AIF. Conversely, the AIF could blur an existing correlation in the model with respect to $\mathrm{rCBV}$, so that it did not have a visible statistical effect in the model with respect to rCBF anymore.

\section{Predicting Normalized Brain Volume}

Neither the model containing rCBV nor the model containing rCBF contributes significantly to explaining the systematic variability in NBV for gMS. For gCIS both models, that containing rCBV as well as that containing rCBF, contribute significantly to explaining the variation in NBV, but with somewhat different predictors. This argues strongly in favour of a systematic difference between gCIS and gMS with respect to the perfusion metrics, even though this difference is not reflected in the simple group comparisons.

In more detail, mean rCBV of GMCort is a significant predictor for NBV in gCIS, but mean $\mathrm{rCBF}$ of GMCort is not a predictor for NBV. Conversely, both, mean rCBV of WMROI and mean rCBF of WMROI are significant predictors for NBV in gCIS. Firstly, this indicates that there is no correlation between either rCBV in GMCort and WMROI or $\mathrm{rCBF}$ in GMCort and WMROI, as such a correlation would render both predictors insignificant. This is consistent with the study of Varga et al. (2009), who did not find a correlation between NAWM and NAGM in terms of perfusion metrics in CIS patients. Secondly, this indicates that ratio of $\mathrm{rCBV}$ and $\mathrm{rCBF}$ is not constant over all ROI in gCIS. Assuming this change in ratios is at least partly driven by a change in perfusion, these findings are generally consistent with the studies of Law et al. (2004) and Varga et al. (2009), who both found perfusion changes in CBF but not in CBV. However, it is worth noting that while the findings of this study support the idea of a different development in $\mathrm{rCBF}$ and $\mathrm{rCBV}$, there is no information on which of the perfusion 
parameters is changing. This study can be interpreted both ways, depending on the sensitivity of NBV to reflect the degree of damage due to the disease. Coming back to the string of arguments presented in Section 4.1, it seems not unlikely that NBV does not (yet) capture the diffuse damage induced by the disease. In this case, it would make perfect sense for the correlation of $\mathrm{rCBF}$ and $\mathrm{NBV}$ to break down, if $\mathrm{rCBF}$ is being affected while NBV is not. This notion is supported by the fact that all correlations break down in gMS, suggesting that the development is towards less association in more severely affected patients. It is also in line with the results of previous studies, which found that rCBF was affected in CIS and RRMS patients, while rCBV remained unaffected (Law et al. 2004; Varga et al. 2009).

For deep GM, only mean rCBV of THAL as well as mean rCBF of THAL for gCIS are significant predictors for NBV. Mean rCBV as well as mean rCBF of PUT are not significant predictors for NBV in gCIS. This argues in favour of a general difference between the perfusion in THAL and PUT. Coming back to the argument that a breakdown of the correlation with NBV represents a further advanced change, the results are in line with the idea of Varga et al. (2009) that the putamen is affected by changes in perfusion before the thalamus. However, the results presented here run counter to those of Papadaki et al. (2014b), who found all CIS patients included in their study to display elevated CBV and reduced CBF in the thalamus compared to healthy controls, suggesting that thalamus perfusion is also already affected in CIS patients. Furthermore, the existing correlation between mean $\mathrm{rCBV}$ and $\mathrm{rCBF}$ of THAL, respectively, and NBV argues against the idea that there is a significant change in one perfusion metric but not in the other.

Gender is a significant predictor for NBV in gCIS in the model with respect to $\mathrm{rCBV}$, but not in the model with respect to $\mathrm{rCBF}$. Gender is known to have an effect on NBV (Luders et al. 2005), so it would be expected to be a significant predictor. But, more importantly, since both models are based on the same dataset where gender and NBV are concerned, there is no conceivable reason why gender would be correlated to NBV in one case but not in the other. The most likely reason for this behaviour is an existing correlation between gender and another predictor in the model with respect to $\mathrm{rCBF}$. And since all non-perfusion-related predictors are identical in both models, this would have to be a correlation between gender and mean $\mathrm{rCBF}$ for at least one of the ROIs, GMCort or PUT, which do not show up as significant predictors. Assuming an 
underlying correlation to be responsible for this behaviour, GMCort is the most likely candidate.

DisDur is not a significant predictor of NBV, regardless of the perfusion metric. This is consistent behaviour, since both models represent exactly the same dataset with respect to DisDur and NBV. However, brain volume loss has been shown to proceed at a significantly higher rater per year in RRMS patients than in healthy controls (Simon 2006; Fotenos et al. 2008; Fisher et al. 2008; Barkhof et al. 2009; De Stefano et al. 2010; De Stefano et al. 2014; Vollmer et al. 2015), so a correlation between NBV and DisDur is an expected finding. There are two reasons for the absence of this correlation. Firstly, there could be a correlation between DisDur and mean rCBV or mean rCBF, respectively, for at least one of the ROI, which are not significant predictors. This is possible, but not likely, compare Figure 3.3. Also, Varga et al. (2009) found no correlation between DisDur and either perfusion metrics. Secondly, the reason could simply be that NBV does not (yet) reflect systematic disease-induced brain volume loss in the data underpinning this study, as explained above. This the most likely reason for the absence of a correlation between DisDur and NBV.

Age is a significant predictor in case of $\mathrm{rCBF}$, but not in case of $\mathrm{rCBV}$. This is counterintuitive at first glance, as age is known to be correlated to brain volume loss (e.g. Simon 2006; Fotenos et al. 2008; Fisher et al. 2008; Barkhof et al. 2009; De Stefano et al. 2010; De Stefano et al. 2014; Vollmer et al. 2015). Also, both models are based on exactly the same dataset where age and NBV are concerned, so either age is correlated NBV or it is not. However, it could simply be a statistical effect induced by a weak correlation between age and either of the non-significant predictors in the model with respect to rCBV. If such an existing correlation was sufficiently distorted by the AIFs, age could become a significant predictor in the model with respect to $\mathrm{rCBF}$ in turn.

When comparing the respective models for the two subject subgroups, gCIS and gMS, the results convincingly indicate that there is a difference in perfusion between gCIS and gMS. This seems to contradict the results from the simple group comparisons. But it probably only reflects that the differences in perfusion are too subtle and possibly also too local to be captured by simply comparing group medians. However, it is not always straightforward to translate the regression results to differences between the two subject subgroups for specific ROI. In case of PUT, neither mean rCBV nor mean rCBF are correlated with NBV. Superficially this differs from the findings of Varga 
et al. (2009) and Papadaki et al. (2012), who found CBF in the putamen to be reduced in RRMS patients compared to CIS patients. But keeping in mind that NBV is not likely to capture the disease-induced change (yet), the absence of a correlation in both models only implies that perfusion in the putamen is affected in both groups. However, it does not imply that putamen perfusion in the respective group, gCIS and gMS, is affected to the same degree. So the results of the presented do not contradict previous findings. In case of THAL, both perfusion metrics are correlated to NBV in gCIS, while none of the perfusion metrics is correlated to NBV in gMS. This could be due to correlations between the perfusion metrics of different ROIs, which only exist in gMS, but it more likely presents a systematic difference with respect to thalamus perfusion between gCIS and gMS. As discussed above, the existing literature is inconsistent a possible difference between CIS patients and RRMS patients in thalamus perfusion, so the results presented here fit in reasonably well. In view of the idea of a spreading change in perfusion (Varga et al. 2009), these findings are consistent with an ongoing process in the thalamus. However, it is impossible to derive only from these results, whether the effect in our models is caused by more severe change in gMS. But accepting the string of arguments presented above that NBV does not (yet) sufficiently reflect the changes induced by the disease, the model results indicate that thalamus perfusion is only affected as the disease progresses as Varga et al. (2009) suggested. The same arguments hold true for the perfusion of WMROI, which exhibits the same behaviour as the perfusion of THAL with respect to our models. There is evidence that perfusion in NAWM is more severly affected in RRMS patients than in CIS patients (Papadaki et al. 2012), which is consistent with the presented findings. However, there is also evidence that WM perfusion is already affected in CIS patients (Papadaki et al. 2012). But if NBV is not capturing the disease-induced damage, an already disturbed WM perfusion should also destroy a correlation between WMROI perfusion and NBV in gCIS. Therefore there is not much support in the results of the regression models for the idea of Varga et al. (2009) that NAWM is the first region to show relevant perfusion changes. But to put that into perspective, most studies found changes (only) in specific subregions of NAWM. Since WMROI also comprises the comparatively large part of still only lightly affected tissue, the early change in WM perfusion might be concealed in the regression models used in this study.

Interestingly, pooling all data results in a model which comprises more significant in- 
dividual predictors for both perfusion metrics, $\mathrm{rCBV}$ and $\mathrm{rCBF}$, than a simple union of both sets of significant predictors from the subgroup models would contain. This suggests that pooling the data either uncovers more correlations between the respective predictors and NBV, or that pooling distorts existing correlations between predictors enough to make these predictors assume statistical significance. Superficially, this also seems similar to the results of Varga et al. (2009), who did not find a correlation between NAWM and NAGM perfusion metrics for either CIS patients or MS patients, but found such a correlation in the pooled data of all patients. However, the findings presented here run counter to the findings of Varga et al. (2009) on closer inspection, as more pronounced correlations between the predictors would lead to a smaller number of significant predictors in the regression model for all pooled data.

Generally, the results presented here argue in favour of a difference in perfusion metrics and/or internal correlations between gCIS and gMS, which is in line with the discussed earlier studies, which is in line with the results of studies, which directly compared CIS patients and RRMS patients (Varga et al. 2009; Papadaki et al. 2012). But it should be kept in mind that those differences were derived from group comparisons, not from the existence of correlations. In terms of correlations, several studies found an inverse correlation between verbal memory and executive motor task function and elevated CBV values in NAWM and deep grey matter (Papadaki et al. 2012; Papadaki et al. 2014a; Papadaki et al. 2014b). Additionally, Inglese et al. (2007) found that both, CBV and CBF, correlated with the fatigue score of RRMS patients. None of the existing perfusions studies using DSC imaging found relevant correlations between perfusion metrics and any volumetric parameters such as NBV. A possible reason for this is the use of different statistical methods to investigate possible correlations. Interestingly, Marshall et al. (2014) not only found an inverse correlation between GM cerebrovascular reactivity and lesion volume, but also a correlation between GM cerebrovascular reactivity and GM atrophy using ASL imaging. So there might be other methodically issues apart from statistics, which should be investigated in future studies.

\section{Predicting Expanded Disability Status Scale}

Neither the model with respect to rCBV nor the model with respect torCBF contributes significantly to explaining the systematic variability in EDSS for either gCIS or gMS, respectively. Pooling all data does not improve the models in terms of significant pre- 
dictors. This is in line with the results of Varga et al. (2009) and Papadaki et al. (2012), who did not find any correlations between perfusion metrics EDSS scores for either CIS or RRMS patients. However, the results presented here do not necessarily mean that there is no correlation between changes in perfusion metrics and progressing disability. Although EDSS is a widely used measure for clinical disability, it is comparatively unsuitable as a measure of clinical disease progression in statistical studies as it is not continuous. With regard to content, using the EDSS it is not possible to discriminate disability on any finely grained scale, which also makes its suitability in the context of this and other comparable studies questionable. Furthermore, using a (single) number to quantify the degree to which a patient is clinically affected might seem absolutely objective, but it is not. For one thing, the EDSS does not at all reflect, which part of the nervous system is affected. Therefore it is only marginally useful in trying to attribute effects to a specific region and/or system. Furthermore, the EDSS attributes disproportionately high weight to the ability to walk. This makes perfect sense in a clinical context when judging disability in its relevance to daily life. But for generally analysing the progress of disability in all different functional systems and the potentially regional influences on this change, it would make more sense to weigh every all functional systems evenly - or alternatively use measures, which are less compressed than EDSS. Additionally, two patients can score the same EDSS while one is moderately to severely affected in one functional system and the other is only slightly affected in several functional system. Again, this makes sense in a clinical context with respect to functional disability. When analysing the different functional systems, both, severity of the disability and the affected functional system is most relevant information, which the EDSS cannot always provide. For all these reasons, the EDSS is rather unlikely to sufficiently represent the effect which should be investigated. Therefore the lack of any significant correlation in all models with respect to EDSS does not indicate there is no correlation between the progress of disability and a change in perfusion metrics in defined regions of the brain.

\subsubsection{Responsible mechanisms}

The mechanisms responsible for the change in perfusion in CIS and MS patients are not yet fully understood. Ge et al. (2005b) and Wuerfel et al. (2007) suggested vasodi- 
lation and/or angiogenesis induced by inflammation as the mechanism responsible for elevated CBV in lesions of CIS patients. Peruzzo et al. (2012) found some instances of inflammation-induced hyperperfusion in cortical lesions, but mostly they found cortical lesions to display a decrease in perfusion. They suggested a reduction of metabolism because of the loss of cortical neurons as a main reason for this reduction in perfusion (Peruzzo et al. 2012). Contrary to that, Saindane et al. (2007) suggested that the change in perfusion is driven by primary ischemia rather than decreased perfusion induced by axonal damage. They based their suggestions on the finding that decreased CBF positively correlated with mean diffusivity but not with fractional anisotropy (Saindane et al. 2007). Moreover, Debernard et al. (2014) found that RRMS patients who did not yet show any volume loss indicative of axonal loss already displayed reduced cortical and deep gray matter CBF. This does not discount neuronal metabolic dysfunction as a reason for changes in perfusion, but it argues against the idea of axonal loss being the reason for this change in metabolism.

However, changes in perfusion are not limited to lesions, but are a widespread finding in MS. There is no evidence that structural abnormalities of small blood vessels such as microthrombosis are a common occurrence in MS lesions (De Keyser et al. 2008), more or less discounting this as the mechanism responsible for decreased perfusion in cortical lesions. Varga et al. (2009) suggested that a continuum of tissue perfusion decrease due to inflammatory processes is responsible for the differences in perfusion between CIS and MS patients, spreads from white to grey matter as the disease progresses. Both assumptions were in some fashion corroborated by Lucchinetti et al. (2011), who found widespread cortical and even meningeal inflammation in cerebral biopsies of early MS patients. Data from two further studies (Law et al. 2004, Papadaki et al. 2014a; Papadaki et al. 2014b) supported the view that hemodynamic change is induced by diffuse and widespread inflammatory processes in the respective brain regions. Papadaki et al. (2012) suggested that vasodilatation and/or angiogenesis induced by inflammation are likely also responsible for elevated CBV in several normal appearing regions of the brains of CIS patients. However, the results of Marshall et al. (2014) showed a reduced dilatory capacity of cerebral arterioles in response to vasomotor stimulation MS patients compared to healthy controls using ASL imaging. This indicates that vasodilatation might not be a key player in perfusion change in MS patients, but at the same time it emphasises that cerebrovascular reactivity is affected in MS. Vessel pathology has 
received increasing attention as a potential reason for diffuse pathology in the last couple of years. Several studies found enhanced levels of the potent vasoconstrictive peptide ET-1 in the blood and some also in the cerebrospinal fluid in MS patients (Speciale et al. 2000; Haufschild et al. 2001; Pache et al. 2003). Moreover, D'haeseleer et al. (2013) found a $20 \%$ reduction of CBF in MS patients compared to healthy controls to be reversible after administration of a ET-1 receptor antagonist, which strongly indicates a causal relationship. D'haeseleer et al. (2015) also deduced that the release of ET-1 in the cerebral circulation of MS patients is likely responsible for inducing arteriolar vasoconstriction.

Generally, there is nothing in the data underpinning the study presented here to indicate any of these assumptions could be unfounded. In view of previous findings, inflammation and also ET-1 release still seem the most likely reasons for hemodynamic changes in CIS and MS patients. There is some evidence in our regression models that the change in cerebral perfusion in MS is an ongoing process, which affects almost all of the ROIs. This supports the theory of Varga et al. (2009), but not all of the findings presented here are consistent with that theory.

\subsection{Conclusions and Outlook}

Among the limitations of this study are its cross-sectional nature and the use of the comparatively coarse measures of disease severity and brain damage, EDSS and NBV. The use of DSC imaging also comes based on some assumptions and models. And while manual definition of the AIF is superior to automatic determination, basing the definition of the AIF on only four voxels is also a potential source of uncertainty.

In conclusion, there were no simple group differences in perfusion metrics between CIS and MS patients for the various white matter and grey matter ROIs. However, there is some evidence for existing differences in perfusion not only between CIS and MS patients, but also between several different (grey) matter ROIs within each group. Especially, the putamen displays disparate behaviour. The results of this study indicate that there are relevant hemodynamic changes early on in the course of the disease, as CIS patients show a different behaviour from the cohort of RRMS patients, even though the RRMS cohort mostly features comparatively short individual disease duration. Generally, the findings of this study suggest that the underlying mechanism responsible for 
hemodynamic changes is diffuse rather than focal and seems to be subject to some sort of (temporal) evolution, as changes do not seem to occur simultaneously in all regions in the brain. But because of the limited accuracy of calculating NBV, the results are open to several interpretations with respect to the sequence in which the different regions are affected. The results further suggest that hemodynamic changes might be a much more sensitive marker than previously thought. Moreover, this study indicates that there is likely little merit in simply comparing averaged perfusion metrics per ROI as this methods lacks the necessary sensitivity. The crucial information is most likely to be had from simultaneous correlation analyses.

Ideally, longitudinal studies with higher resolution ROIs are needed to further investigate the role of hemodynamic change at different stages of the disease and to evaluate the changing patterns of correlations. Known correlations between hemodynamic change and higher brain functions and neuropsychological functions, such as parts of the memory and fatigue, should be more thoroughly investigated with respect to a potential causal relationship. Considerings that possibly a continuous process is driving the change in perfusion, these correlations assume a new significance not only with respect to reliable markers for disease progression but also with respect to possible predictors of disease progression. But to fully utilise these presumed relationships, it is necessary to further scientific understanding of the mechanisms responsible for hemodynamic change, particularly on a microscopic scale.

Finally, perfusion metrics deserve some attention with respect to a pharmacological point of view. Not least, changes in perfusion metric show promising potential as a very sensitive marker for drug efficacy in MS treatment. But even more importantly, the potential response to treatment merits scientific efforts. If hemodynamic change is a slowly evolving process which starts early on in the course of the disease, it might present a suitable drug target. This is especially important, as the axonal damage which can occur in the wake of hypoperfusion even makes it a potential drug target in the so far elusive progressive forms of MS. 


\section{Summary}

A total of 106 untreated patients aged between 18 and 65, who had been diagnosed with clinically isolated syndrome (CIS) or relapsing-remitting multiple sclerosis (RRMS) were prospectively recruited, of which 95 patients were finally included in the study presented here. All patients were evaluated according to the Expanded Disability Status Scale (EDSS) and underwent MRI including perfusion imaging. Normalized brain volume (NBV), average relative cerebral blood volume (rCBV) and average relative cerebral blood flow $(\mathrm{rCBF})$ were obtained for non-lesional tissue of the thalamus, the putamen, the cortical grey matter and the white matter. Subjects were dichotomised according to their diagnosis.

The two groups are similar with respect to demographic and disease characteristics except for a small difference in disease duration. There is no significant difference between both subject subgroups with respect to regional volumes or perfusion parameters, respectively, in any of the regions of interest (ROIs). The relations between pairs of ROIs within each subject subgroup are heterogeneous. Perfusion parameters are not correlated with EDSS in any of the two subgroups. Perfusion parameters are not correlated with NBV in the subgroup of RRMS patients. Mean rCBV of the GM, of the thalamus and of the WM is correlated with NBV for the subgroup of CIS patients, but only rCBF of the thalamus and of the WM is correlated to NBV.

The results from the regression models indicate that there is a difference in local perfusion between CIS and RRMS patients, even though the RRMS cohort mostly features comparatively short individual disease duration, which is largely consistent with the literature. The fact that these differences are not reflected in the results of the simple group comparisons is likely due to its comparatively small magnitude. Especially, this study indicates that there is likely little merit in simply comparing averaged perfusion metrics per ROI as this methods lacks the necessary sensitivity. The crucial information 
is most likely to be had from simultaneous correlation analyses. Beside the comparatively coarse measures of disease severity and brain damage, EDSS and NBV, this study is limited by its cross-sectional nature.

In summary, the results of this study suggest that the underlying mechanism responsible for hemodynamic changes is diffuse rather than focal and is subject to some sort of (temporal) evolution. But because of the limited accuracy of calculating NBV, the results are open to several interpretations with respect to the sequence in which the different regions are affected. Ideally, longitudinal studies with higher resolution ROIs are needed to further investigate the role of hemodynamic change at different stages of the disease and to evaluate the changing patterns of correlations. 


\section{Bibliography}

Adams CW (1988): Perivascular iron deposition and other vascular damage in multiple sclerosis. J Neurol Neurosurg Psychiatr 51 (2), 260-265

Adams CW, Poston RN, Buk SJ, Sidhu YS, Vipond H (1985): Inflammatory vasculitis in multiple sclerosis. J Neurol Sci 69 (3), 269-283

Adhya S, Johnson G, Herbert J, Jaggi H, Babb JS, Grossman RI, Inglese M (2006): Pattern of hemodynamic impairment in multiple sclerosis: dynamic susceptibility contrast perfusion MR imaging at 3.0 t. NeuroImage 33 (4), 1029-1035

Alotaibi S, Kennedy J, Tellier R, Stephens D, Banwell B (2004): Epstein-Barr virus in pediatric multiple sclerosis. JAMA 291 (15), 1875-1879

Amato MP, Ponziani G, Pracucci G, Bracco L, Siracusa G, Amaducci L (1995): Cognitive impairment in early-onset multiple sclerosis. Pattern, predictors, and impact on everyday life in a 4-year follow-up. Arch Neurol $\underline{52}$ (2), 168-172

Amato MP, Ponziani G, Siracusa G, Sorbi S (2001): Cognitive dysfunction in early-onset multiple sclerosis: a reappraisal after 10 years. Arch Neurol 58 (10), 1602-1606

Amato MP, Grimaud J, Achiti I, Bartolozzi ML, Adeleine P, Hartung HP, Kappos L, Thompson A, Trojano M, Vukusic S (2004): European validation of a standardized clinical description of multiple sclerosis. J Neurol 251 (12), 1472-1480

Amato MP, Portaccio E, Goretti B, Zipoli V, Battaglini M, Bartolozzi ML, Stromillo ML, Guidi L, Siracusa G, Sorbi S (2007): Association of neocortical volume changes with cognitive deterioration in relapsing-remitting multiple sclerosis. Arch Neurol $\underline{64}(8), 1157-1161$ 
Anderson VM, Fox NC, Miller DH (2006): Magnetic resonance imaging measures of brain atrophy in multiple sclerosis. J Magn Reson Imaging 23 (5), 605-618

Androdias G, Reynolds R, Chanal M, Ritleng C, Confavreux C, Nataf S (2010): Meningeal T cells associate with diffuse axonal loss in multiple sclerosis spinal cords. Ann Neurol 68 (4), 465-476

Ascherio A, Munch M (2000): Epstein-Barr virus and multiple sclerosis. Epidemiology $\underline{11}(2), 220-224$

Ascherio A, Munger KL (2007a): Environmental risk factors for multiple sclerosis. Part I: the role of infection. Ann Neurol 61 (4), 288-299

Ascherio A, Munger KL (2007b): Environmental risk factors for multiple sclerosis. Part II: Noninfectious factors. Ann Neurol 61 (6), 504-513

Ascherio A, Munger KL, Lennette ET, Spiegelman D, Hernán MA, Olek MJ, Hankinson SE, Hunter DJ (2001): Epstein-Barr virus antibodies and risk of multiple sclerosis: a prospective study. JAMA 286 (24), 3083-3088

Bach JF (2002): The effect of infections on susceptibility to autoimmune and allergic diseases. N Engl J Med 347 (12), 911-920

Barkhof F (2002): The clinico-radiological paradox in multiple sclerosis revisited. Curr Opin Neurol 15 (3), 239-245

Barkhof F, Calabresi PA, Miller DH, Reingold SC (2009): Imaging outcomes for neuroprotection and repair in multiple sclerosis trials. Nat Rev Neurol $\underline{5}$ (5), 256-266

Barnett MH, Prineas JW (2004): Relapsing and remitting multiple sclerosis: pathology of the newly forming lesion. Ann Neurol 55 (4), 458-468

Barten LJ, Allington DR, Procacci KA, Rivey MP (2010): New approaches in the management of multiple sclerosis. Drug Des Devel Ther 4, 343-366

Bartolomei I, Salvi F, Galeotti R, Salviato E, Alcanterini M, Menegatti E, Mascalchi M, Zamboni P (2010): Hemodynamic patterns of chronic cerebrospinal venous insufficiency in multiple sclerosis. Correlation with symptoms at onset and clinical course. Int Angiol 29 (2), 183-188 
Bedekar D, Jensen T, Schmainda KM (2010): Standardization of relative cerebral blood volume (rCBV) image maps for ease of both inter- and intrapatient comparisons. Magn Reson Med 64 (3), 907-913

Bermel RA, Bakshi R (2006): The measurement and clinical relevance of brain atrophy in multiple sclerosis. Lancet Neurol $\underline{5}$ (2), 158-170

Bjartmar C, Kidd G, Mörk S, Rudick R, Trapp BD (2000): Neurological disability correlates with spinal cord axonal loss and reduced N-acetyl aspartate in chronic multiple sclerosis patients. Ann Neurol 48 (6), 893-901

Bö L, Geurts JJG, Mörk SJ, van der Valk P (2006): Grey matter pathology in multiple sclerosis. Acta Neurol Scand, Suppl 183, 48-50

Bourdette DN, Cohen JA (2014): Venous angioplasty for "CCSVI" in multiple sclerosis: ending a therapeutic misadventure. Neurology 83 (5), 388-389

Boxerman JL, Schmainda KM, Weisskoff RM (2006): Relative cerebral blood volume maps corrected for contrast agent extravasation significantly correlate with glioma tumor grade, whereas uncorrected maps do not. AJNR Am J Neuroradiol 27 (4), 859-867

Brex PA, Leary SM, Plant GT, Thompson AJ, Miller DH (2001): Magnetization transfer imaging in patients with clinically isolated syndromes suggestive of multiple sclerosis. AJNR Am J Neuroradiol 22 (5), 947-951

Calabresi PA, Radue EW, Goodin D, Jeffery D, Rammohan KW, Reder AT, Vollmer T, Agius MA, Kappos L, Stites T (2014): Safety and efficacy of fingolimod in patients with relapsing-remitting multiple sclerosis (FREEDOMS II): a double-blind, randomised, placebo-controlled, phase 3 trial. Lancet Neurol 13 (6), 545-556

Cantorna MT, Hayes CE, DeLuca HF (1996): 1,25-Dihydroxyvitamin D3 reversibly blocks the progression of relapsing encephalomyelitis, a model of multiple sclerosis. Proc Natl Acad Sci U S A 93 (15), 7861-7864

Cardaioli G, Di Filippo M, Bianchi A, Eusebi P, Di Gregorio M, Gaetani L, Gallina A, Calabresi P, Sarchielli P (2016): Extracranial Venous Drainage Pattern in Multi- 
ple Sclerosis and Healthy Controls: Application of the 2011 Diagnostic Criteria for Chronic Cerebrospinal Venous Insufficiency. Eur Neurol 76 (1-2), 62-68

Casanova B, Martínez-Bisbal MC, Valero C, Celda B, Martí-Bonmatí L, Pascual A, Landente L, Coret F (2003): Evidence of Wallerian degeneration in normal appearing white matter in the early stages of relapsing-remitting multiple sclerosis: a HMRS study. J Neurol 250 (1), 22-28

Chard DT, Miller DH (2009): Grey matter pathology in clinically early multiple sclerosis: evidence from magnetic resonance imaging. J Neurol Sci $\underline{282}$ (1-2), 5-11

Chard DT, Griffin CM, Parker GJM, Kapoor R, Thompson AJ, Miller DH (2002): Brain atrophy in clinically early relapsing-remitting multiple sclerosis. Brain 125, 327-337

Chard DT, Griffin CM, Rashid W, Davies GR, Altmann DR, Kapoor R, Barker GJ, Thompson AJ, Miller DH (2004): Progressive grey matter atrophy in clinically early relapsing-remitting multiple sclerosis. Mult Scler 10 (4), 387-391

Chiaravalloti ND, DeLuca J (2008): Cognitive impairment in multiple sclerosis. Lancet Neurol 7 (12), 1139-1151

Cohen JA, Coles AJ, Arnold DL, Confavreux C, Fox EJ, Hartung HP, Havrdova E, Selmaj KW, Weiner HL, Fisher E (2012): Alemtuzumab versus interferon beta 1a as first-line treatment for patients with relapsing-remitting multiple sclerosis: a randomised controlled phase 3 trial. Lancet 380 (9856), 1819-1828

Comi G, Filippi M, Barkhof F, Durelli L, Edan G, Fernández O, Hartung H, Seeldrayers P, Sørensen PS, Rovaris M (2001): Effect of early interferon treatment on conversion to definite multiple sclerosis: a randomised study. Lancet 357 (9268), 1576-1582

Comi G, Martinelli V, Rodegher M, Moiola L, Bajenaru O, Carra A, Elovaara I, Fazekas F, Hartung HP, Hillert J (2009): Effect of glatiramer acetate on conversion to clinically definite multiple sclerosis in patients with clinically isolated syndrome (PreCISe study): a randomised, double-blind, placebo-controlled trial. Lancet 374 (9700), $1503-1511$

Compston A (1999): The genetic epidemiology of multiple sclerosis. Philos Trans R Soc Lond, B, Biol Sci 354 (1390), 1623-1634 
Compston A, Coles A (2002): Multiple sclerosis. Lancet 359 (9313), 1221-1231

Confavreux C, Vukusic S (2006): Age at disability milestones in multiple sclerosis. Brain $\underline{129}, 595-605$

Conte A, Lenzi D, Frasca V, Gilio F, Giacomelli E, Gabriele M, Bettolo CM, Iacovelli E, Pantano P, Pozzilli C (2009): Intracortical excitability in patients with relapsingremitting and secondary progressive multiple sclerosis. J Neurol 256 (6), 933-938

Conturo TE, Akbudak E, Kotys MS, Chen ML, Chun SJ, Hsu RM, Sweeney CC, Markham J (2005): Arterial input functions for dynamic susceptibility contrast MRI: requirements and signal options. J Magn Reson Imaging 22 (6), 697-703

Dale A, Sereno MI (1993): Improved localizadon of cortical activity by combining eeg and meg with mri cortical surface reconstruction: A linear approach. J Cogn Neurosci $\underline{5}(2), 162-176$

Dale A, Fischl B, Sereno MI (1999): Cortical surface-based analysis: I. segmentation and surface reconstruction. NeuroImage 9 (2), 179-194

De Keyser J, Steen C, Mostert JP, Koch MW (2008): Hypoperfusion of the cerebral white matter in multiple sclerosis: possible mechanisms and pathophysiological significance. J Cereb Blood Flow Metab 28 (10), 1645-1651

De Stefano N, Matthews PM, Filippi M, Agosta F, De Luca M, Bartolozzi ML, Guidi L, Ghezzi A, Montanari E, Cifelli A (2003): Evidence of early cortical atrophy in MS: relevance to white matter changes and disability. Neurology $\underline{60}$ (7), 1157-1162

De Stefano N, Battaglini M, Smith SM (2007): Measuring brain atrophy in multiple sclerosis. J Neuroimaging 17 (Suppl 1), 10S-15S

De Stefano N, Giorgio A, Battaglini M, Rovaris M, Sormani MP, Barkhof F, Korteweg T, Enzinger C, Fazekas F, Calabrese M (2010): Assessing brain atrophy rates in a large population of untreated multiple sclerosis subtypes. Neurology 74 (23), 1868-1876

De Stefano N, Airas L, Grigoriadis N, Mattle HP, O'Riordan J, Oreja-Guevara C, Sellebjerg F, Stankoff B, Walczak A, Wiendl H (2014): Clinical relevance of brain volume measures in multiple sclerosis. CNS Drugs 28 (2), 147-156 
Debernard L, Melzer TR, Van Stockum S, Graham C, Wheeler-Kingshott CA, Dalrymple-Alford JC, Miller DH, Mason DF (2014): Reduced grey matter perfusion without volume loss in early relapsing-remitting multiple sclerosis. J Neurol Neurosurg Psychiatry 85 (5), 544-551

D'haeseleer M, Beelen R, Fierens Y, Cambron M, Vanbinst AM, Verborgh C, Demey J, De Keyser J (2013): Cerebral hypoperfusion in multiple sclerosis is reversible and mediated by endothelin-1. Proc Natl Acad Sci U S A 110 (14), 5654-5658

D'haeseleer M, Hostenbach S, Peeters I, Sankari SE, Nagels G, De Keyser J, D'hooghe MB (2015): Cerebral hypoperfusion: a new pathophysiologic concept in multiple sclerosis? J Cereb Blood Flow Metab 35 (9), 1406-1410

Doepp F, Paul F, Valdueza JM, Schmierer K, Schreiber SJ (2010): No cerebrocervical venous congestion in patients with multiple sclerosis. Ann Neurol $\underline{68}$ (2), 173-183

Doepp F, Würfel JT, Pfueller CF, Valdueza JM, Petersen D, Paul F, Schreiber SJ (2011): Venous drainage in multiple sclerosis: a combined MRI and ultrasound study. Neurology 77 (19), 1745-1751

Dutta R, Trapp BD (2007): Pathogenesis of axonal and neuronal damage in multiple sclerosis. Neurology 68 (22), S22-S31

Dutta R, Trapp BD (2011): Mechanisms of neuronal dysfunction and degeneration in multiple sclerosis. Prog Neurobiol 93 (1), 1-12

Dutta R, McDonough J, Yin X, Peterson J, Chang A, Torres T, Gudz T, Macklin WB, Lewis DA, Fox RJ (2006): Mitochondrial dysfunction as a cause of axonal degeneration in multiple sclerosis patients. Ann Neurol 59 (3), 478-489

Dziedzic T, Metz I, Dallenga T, König FB, Müller S, Stadelmann C, Brück W (2010): Wallerian degeneration: a major component of early axonal pathology in multiple sclerosis. Brain Pathol 20 (5), 976-985

Ebers GC, Koopman WJ, Hader W, Sadovnick AD, Kremenchutzky M, Mandalfino P, Wingerchuk DM, Baskerville J, Rice GP (2000): The natural history of multiple sclerosis: a geographically based study: 8: familial multiple sclerosis. Brain $\underline{123}(\mathrm{Pt}$ 3), 641-649 
Ebinger M, Brunecker P, Jungehülsing GJ, Malzahn U, Kunze C, Endres M, Fiebach JB (2010): Reliable perfusion maps in stroke MRI using arterial input functions derived from distal middle cerebral artery branches. Stroke 41 (1), 95-101

Eggert LD, Sommer J, Jansen A, Kircher T, Konrad C (2012): Accuracy and reliability of automated gray matter segmentation pathways on real and simulated structural magnetic resonance images of the human brain. PloS One 7 (9), e45081

Enzinger C, Fazekas F, Matthews PM, Ropele S, Schmidt H, Smith S, Schmidt R (2005): Risk factors for progression of brain atrophy in aging: six-year follow-up of normal subjects. Neurology 64 (10), 1704-1711

Epplen C, Jäckel S, Santos EJ, D'Souza M, Poehlau D, Dotzauer B, Sindern E, Haupts M, Rüde KP, Weber F (1997): Genetic predisposition to multiple sclerosis as revealed by immunoprinting. Ann Neurol $\underline{41}$ (3), 341-352

Eriksson M, Ben-Menachem E, Andersen O (2002): Epileptic seizures, cranial neuralgias and paroxysmal symptoms in remitting and progressive multiple sclerosis. Mult Scler 8 (6), 495-499

European Study Group on Interferon $\beta-1 b$ in Secondary Progressive MS (1998): Placebo-controlled multicentre randomised trial of interferon beta- $1 \mathrm{~b}$ in treatment of secondary progressive multiple sclerosis. Lancet 352 (9139), 1491-1497

Fellows K, Uher T, Browne RW, Weinstock-Guttman B, Horakova D, Posova H, Vaneckova M, Seidl Z, Krasensky J, Tyblova M (2015): Protective associations of HDL with blood-brain barrier injury in multiple sclerosis patients. J Lipid Res 56 (10), 2010-2018

Ferguson B, Matyszak MK, Esiri MM, Perry VH (1997): Axonal damage in acute multiple sclerosis lesions. Brain 120 (Pt 3), 393-399

Filippi M, Rocca MA (2011): MR imaging of multiple sclerosis. Radiology 259 (3), 659-681

Filippi M, Rocca MA, Barkhof F, Brück W, Chen JT, Comi G, DeLuca G, De Stefano N, Erickson BJ, Evangelou N (2012): Association between pathological and MRI findings in multiple sclerosis. Lancet Neurol 11 (4), 349-360 
Filippi M, Rocca MA, Ciccarelli O, De Stefano N, Evangelou N, Kappos L, Rovira A, Sastre-Garriga J, Tintorè M, Frederiksen JL (2016): MRI criteria for the diagnosis of multiple sclerosis: MAGNIMS consensus guidelines. Lancet Neurol 15 (3), 292-303

Fischl B, Dale AM (2000): Measuring the thickness of the human cerebral cortex from magnetic resonance images. Proc Natl Acad Sci U S A 97 (20), 11050-11055

Fischl B, Sereno MI, Dale A (1999a): Cortical surface-based analysis: II: Inflation, flattening, and a surface-based coordinate system. NeuroImage $\underline{9}$ (2), 195-207

Fischl B, Sereno MI, Tootell RB, Dale AM (1999b): High-resolution intersubject averaging and a coordinate system for the cortical surface. Hum Brain Mapp $\underline{8}$ (4), $272-284$

Fischl B, Liu A, Dale AM (2001): Automated manifold surgery: constructing geometrically accurate and topologically correct models of the human cerebral cortex. IEEE Trans Med Imaging 20 (1), 70-80

Fischl B, Salat DH, Busa E, Albert M, Dieterich M, Haselgrove C, van der Kouwe A, Killiany R, Kennedy D, Klaveness S (2002): Whole brain segmentation: automated labeling of neuroanatomical structures in the human brain. Neuron 33 (3), 341-355

Fischl B, Salat DH, van der Kouwe AJ, Makris N, Ségonne F, Quinn BT, Dale AM (2004a): Sequence-independent segmentation of magnetic resonance images. NeuroImage 23 (Supplement 1), S69-S84

Fischl B, van der Kouwe A, Destrieux C, Halgren E, Ségonne F, Salat DH, Busa E, Seidman LJ, Goldstein J, Kennedy D (2004b): Automatically Parcellating the Human Cerebral Cortex. Cereb Cortex 14 (1), 11-22

Fisher E, Lee JC, Nakamura K, Rudick RA (2008): Gray matter atrophy in multiple sclerosis: a longitudinal study. Ann Neurol 64 (3), 255-265

Fisniku LK, Chard DT, Jackson JS, Anderson VM, Altmann DR, Miszkiel KA, Thompson AJ, Miller DH (2008): Gray matter atrophy is related to long-term disability in multiple sclerosis. Ann Neurol 64 (3), 247-254 
Fisniku LK, Altmann DR, Cercignani M, Tozer DJ, Chard DT, Jackson JS, Miszkiel KA, Schmierer K, Thompson AJ, Miller DH (2009): Magnetization transfer ratio abnormalities reflect clinically relevant grey matter damage in multiple sclerosis. Mult Scler 15 (6), 668-677

Fotenos AF, Mintun MA, Snyder AZ, Morris JC, Buckner RL (2008): Brain volume decline in aging: evidence for a relation between socioeconomic status, preclinical Alzheimer disease, and reserve. Arch Neurol 65 (1), 113-120

FreeSurfer (2016): http://surfer.nmr.mgh.harvard.edu/fswiki/FreeSurferMe thodsCitation; accessed on 24/11/2016

Ge Y, Law M, Herbert J, Grossman RI (2005a): Prominent perivenular spaces in multiple sclerosis as a sign of perivascular inflammation in primary demyelination. AJNR Am J Neuroradiol 26 (9), 2316-2319

Ge Y, Law M, Johnson G, Herbert J, Babb JS, Mannon LJ, Grossman RI (2005b): Dynamic susceptibility contrast perfusion MR imaging of multiple sclerosis lesions: characterizing hemodynamic impairment and inflammatory activity. AJNR Am J Neuroradiol 26 (6), 1539-1547

Ge Y, Zohrabian VM, Grossman RI (2008): Seven-Tesla magnetic resonance imaging: new vision of microvascular abnormalities in multiple sclerosis. Arch Neurol 65 (6), $812-816$

Gold R, Kappos L, Arnold DL, Bar-Or A, Giovannoni G, Selmaj K, Tornatore C, Sweetser MT, Yang M, Sheikh SI (2012): Placebo-controlled phase 3 study of oral BG-12 for relapsing multiple sclerosis. N Engl J Med 367 (12), 1098-1107

Haahr S, Plesner AM, Vestergaard BF, Höllsberg P (2004): A role of late Epstein-Barr virus infection in multiple sclerosis. Acta Neurol Scand 109 (4), 270-275

Han X, Jovicich J, Salat D, van der Kouwe A, Quinn B, Czanner S, Busa E, Pacheco J, Albert M, Killiany R (2006): Reliability of MRI-derived measurements of human cerebral cortical thickness: The effects of field strength, scanner upgrade and manufacturer. NeuroImage 32 (1), 180-194 
Haselhorst R, Kappos L, Bilecen D, Scheffler K, Möri D, Radü EW, Seelig J (2000): Dynamic susceptibility contrast MR imaging of plaque development in multiple sclerosis: application of an extended blood-brain barrier leakage correction. J Magn Reson Imaging 11 (5), 495-505

Haufschild T, Shaw SG, Kesselring J, Flammer J (2001): Increased endothelin-1 plasma levels in patients with multiple sclerosis. J Neuroophthalmol 21 (1), 37-38

Henry RG, Shieh M, Okuda DT, Evangelista A, Gorno-Tempini ML, Pelletier D (2008): Regional grey matter atrophy in clinically isolated syndromes at presentation. J Neurol Neurosurg Psychiatr 79 (11), 1236-1244

Hojjat SP, Cantrell CG, Vitorino R, Feinstein A, Shirzadi Z, MacIntosh BJ, Crane DE, Zhang L, Morrow SA, Lee L (2016): Regional reduction in cortical blood flow among cognitively impaired adults with relapsing-remitting multiple sclerosis patients. Mult Scler 22 (11), 1421-1428

Holm S (1979): A simple sequentially rejective multiple test procedure. Scand J Stat $\underline{6}$, $65-70$

Hu LS, Kelm Z, Korfiatis P, Dueck AC, Elrod C, Ellingson BM, Kaufmann TJ, Eschbacher JM, Karis JP, Smith K (2015): Impact of Software Modeling on the Accuracy of Perfusion MRI in Glioma. AJNR Am J Neuroradiol 36 (12), 2242-2249

Hyndman RJ, Fan Y (1996): Sample quantiles in statistical packages. Am Stat 50 (4), $361-365$

Iannucci G, Tortorella C, Rovaris M, Sormani MP, Comi G, Filippi M (2000): Prognostic value of MR and magnetization transfer imaging findings in patients with clinically isolated syndromes suggestive of multiple sclerosis at presentation. AJNR Am J Neuroradiol 21 (6), 1034-1038

Inglese M, Ge Y, Filippi M, Falini A, Grossman RI, Gonen O (2004): Indirect evidence for early widespread gray matter involvement in relapsing-remitting multiple sclerosis. NeuroImage $\underline{21}$ (4), 1825-1829 
Inglese M, Park SJ, Johnson G, Babb JS, Miles L, Jaggi H, Herbert J, Grossman RI (2007): Deep gray matter perfusion in multiple sclerosis: dynamic susceptibility contrast perfusion magnetic resonance imaging at 3 T. Arch Neurol 64 (2), 196-202

Jacobs LD, Beck RW, Simon JH, Kinkel RP, Brownscheidle CM, Murray TJ, Simonian NA, Slasor PJ, Sandrock AW (2000): Intramuscular interferon beta-1a therapy initiated during a first demyelinating event in multiple sclerosis. CHAMPS study group. N Engl J Med 343 (13), 898-904

Jacobsen C, Hagemeier J, Myhr KM, Nyland H, Lode K, Bergsland N, Ramasamy DP, Dalaker TO, Larsen JP, Farbu E (2014): Brain atrophy and disability progression in multiple sclerosis patients: a 10-year follow-up study. J Neurol Neurosurg Psychiatry $\underline{85}$ (10), 1109-1115

Jenkinson M, Smith S (2001): A global optimisation method for robust affine registration of brain images. Med Image Anal $\underline{5}$ (2), 143-156

Jenkinson M, Bannister P, Brady M, Smith S (2002): Improved optimization for the robust and accurate linear registration and motion correction of brain images. NeuroImage $\underline{17}(2), 825-841$

Jenkinson M, Beckmann CF, Behrens TEJ, Woolrich MW, Smith SM (2012): FSL. NeuroImage $\underline{62}(2), 782-790$

Jezzard P, Balaban RS (1995): Correction for geometric distortion in echo planar images from B0 field variations. Magn Reson Med 34 (1), 65-73

Jovicich J, Czanner S, Greve D, Haley E, van der Kouwe A, Gollub R, Kennedy D, Schmitt F, Brown G, MacFall J (2006): Reliability in multi-site structural mri studies: Effects of gradient non-linearity correction on phantom and human data. NeuroImage $\underline{30}(2), 436-443$

Kappos L, Polman CH, Freedman MS, Edan G, Hartung HP, Miller DH, Montalban X, Barkhof F, Bauer L, Jakobs P (2006): Treatment with interferon beta-1b delays conversion to clinically definite and McDonald MS in patients with clinically isolated syndromes. Neurology 67 (7), 1242-1249 
Kappos L, Radue EW, O'Connor P, Polman C, Hohlfeld R, Calabresi P, Selmaj K, Agoropoulou C, Leyk M, Zhang-Auberson L (2010): A placebo-controlled trial of oral fingolimod in relapsing multiple sclerosis. N Engl J Med 362 (5), 387-401

Keegan BM, Noseworthy JH (2002): Multiple sclerosis. Annu Rev Med 53, 285-302

Kidd D, Barkhof F, McConnell R, Algra PR, Allen IV, Revesz T (1999): Cortical lesions in multiple sclerosis. Brain $\underline{122}$ (Pt 1), 17-26

Kipp M, van der Valk P, Amor S (2012): Pathology of multiple sclerosis. CNS Neurol Disord Drug Targets 11 (5), 506-517

Klauschen F, Goldman A, Barra V, Meyer-Lindenberg A, Lundervold A (2009): Evaluation of automated brain MR image segmentation and volumetry methods. Hum Brain Mapp 30 (4), 1310-1327

Kollia K, Maderwald S, Putzki N, Schlamann M, Theysohn JM, Kraff O, Ladd ME, Forsting M, Wanke I (2009): First clinical study on ultra-high-field MR imaging in patients with multiple sclerosis: comparison of 1.5T and 7T. AJNR Am J Neuroradiol $\underline{30}(4), 699-702$

Kornek B, Storch MK, Weissert R, Wallstroem E, Stefferl A, Olsson T, Linington C, Schmidbauer M, Lassmann H (2000): Multiple sclerosis and chronic autoimmune encephalomyelitis: a comparative quantitative study of axonal injury in active, inactive, and remyelinated lesions. Am J Pathol 157 (1), 267-276

Krogias C, Gold R, Chan A, Triantafyllou N, Voumvourakis K, Tsivgoulis G (2016): Brain Hyperechogenicities are not Associated with Venous Insufficiency in Multiple Sclerosis: A Pilot Neurosonology Study. J Neuroimaging 26 (1), 150-155

Kuhlmann T, Lingfeld G, Bitsch A, Schuchardt J, Brück W (2002): Acute axonal damage in multiple sclerosis is most extensive in early disease stages and decreases over time. Brain 125 (10), 2202-2212

Kurtzke JF (1983): Rating neurologic impairment in multiple sclerosis: an expanded disability status scale (EDSS). Neurology 33 (11), 1444-1452 
Kutzelnigg A, Lucchinetti CF, Stadelmann C, Brück W, Rauschka H, Bergmann M, Schmidbauer M, Parisi JE, Lassmann H (2005): Cortical demyelination and diffuse white matter injury in multiple sclerosis. Brain 128, 2705-2712

Law M, Saindane AM, Ge Y, Babb JS, Johnson G, Mannon LJ, Herbert J, Grossman RI (2004): Microvascular abnormality in relapsing-remitting multiple sclerosis: perfusion MR imaging findings in normal-appearing white matter. Radiology 231 (3), 645-652

Leary SM, Thompson AJ (2003): Interferon beta-1a in primary progressive multiple sclerosis. J Neurol Sci 206 (2), 215-216

Leibowitz U, Antonovsky A, Medalie JM, Smith HA, Halpern L, Alter M (1966): Epidemiological study of multiple sclerosis in Israel. II. Multiple sclerosis and level of sanitation. J Neurol Neurosurg Psychiatr 29 (1), 60-68

Lemire JM, Archer DC (1991): 1,25-dihydroxyvitamin D3 prevents the in vivo induction of murine experimental autoimmune encephalomyelitis. J Clin Invest 87 (3), 11031107

Lin R, Charlesworth J, van der Mei I, Taylor BV (2012): The genetics of multiple sclerosis. Pract Neurol 12 (5), 279-288

Lublin FD, Reingold SC (1996): Defining the clinical course of multiple sclerosis: results of an international survey. national multiple sclerosis society (USA) advisory committee on clinical trials of new agents in multiple sclerosis. Neurology 46 (4), $907-911$

Lublin FD, Reingold SC, Cohen JA, Cutter GR, Sørensen PS, Thompson AJ, Wolinsky JS, Balcer LJ, Banwell B, Barkhof F (2014): Defining the clinical course of multiple sclerosis: the 2013 revisions. Neurology 83 (3), 278-286

Lucchinetti CF, Popescu BFG, Bunyan RF, Moll NM, Roemer SF, Lassmann H, Brück W, Parisi JE, Scheithauer BW, Giannini C (2011): Inflammatory cortical demyelination in early multiple sclerosis. N Engl J Med 365 (23), 2188-2197

Luders E, Narr KL, Thompson PM, Woods RP, Rex DE, Jancke L, Steinmetz H, Toga AW (2005): Mapping cortical gray matter in the young adult brain: effects of gender. NeuroImage 26 (2), 493-501 
Lukas C, Minneboo A, de Groot V, Moraal B, Knol DL, Polman CH, Barkhof F, Vrenken H (2010): Early central atrophy rate predicts 5 year clinical outcome in multiple sclerosis. J Neurol Neurosurg Psychiatr 81 (12), 1351-1356

Mandoj C, Renna R, Plantone D, Sperduti I, Cigliana G, Conti L, Koudriavtseva T (2015): Anti-annexin antibodies, cholesterol levels and disability in multiple sclerosis. Neurosci Lett 606, 156-160

Marcus DS, Olsen TR, Ramaratnam M, Buckner RL (2007): The Extensible Neuroimaging Archive Toolkit: an informatics platform for managing, exploring, and sharing neuroimaging data. Neuroinformatics $\underline{5}$ (1), 11-34

Marshall O, Lu H, Brisset JC, Xu F, Liu P, Herbert J, Grossman RI, Ge Y (2014): Impaired cerebrovascular reactivity in multiple sclerosis. JAMA Neurol 71 (10), 12751281

McDonald WI, Compston A, Edan G, Goodkin D, Hartung HP, Lublin FD, McFarland HF, Paty DW, Polman CH, Reingold SC (2001): Recommended diagnostic criteria for multiple sclerosis: guidelines from the international panel on the diagnosis of multiple sclerosis. Ann Neurol 50 (1), 121-127

Mikol DD, Barkhof F, Chang P, Coyle PK, Jeffery DR, Schwid SR, Stubinski B, Uitdehaag BMJ, REGARD study group (2008): Comparison of subcutaneous interferon beta-1a with glatiramer acetate in patients with relapsing multiple sclerosis (the REbif vs glatiramer acetate in relapsing MS disease [REGARD] study): a multicentre, randomised, parallel, open-label trial. Lancet Neurol $\underline{7}$ (10), 903-914

Miller D, Barkhof F, Montalban X, Thompson A, Filippi M (2005a): Clinically isolated syndromes suggestive of multiple sclerosis, part 2: non-conventional MRI, recovery processes, and management. Lancet Neurol 4 (6), 341-348

Miller D, Barkhof F, Montalban X, Thompson A, Filippi M (2005b): Clinically isolated syndromes suggestive of multiple sclerosis, part I: natural history, pathogenesis, diagnosis, and prognosis. Lancet Neurol $\underline{4}$ (5), 281-288

Miller DH, Weinshenker BG, Filippi M, Banwell BL, Cohen JA, Freedman MS, Galetta 
SL, Hutchinson M, Johnson RT, Kappos L (2008): Differential diagnosis of suspected multiple sclerosis: a consensus approach. Mult Scler 14 (9), 1157-1174

Minneboo A, Jasperse B, Barkhof F, Uitdehaag BMJ, Knol DL, de Groot V, Polman CH, Castelijns JA (2008): Predicting short-term disability progression in early multiple sclerosis: added value of MRI parameters. J Neurol Neurosurg Psychiatry $\underline{79}$ (8), 917-923

Mottet I, Quast MJ, Dewitt DS, Hillman GR, Wei J, Uhrbrock DH, Perez-Polo JR, Kent TA (1997): NG-nitro-L-arginine methyl ester modifies the input function measured by dynamic susceptibility contrast magnetic resonance imaging. J Cereb Blood Flow Metab 17 (7), 791-800

Munch M, Hvas J, Christensen T, Møller-Larsen A, Haahr S (1997): The implications of Epstein-Barr virus in multiple sclerosis-a review. Acta Neurol Scand Suppl 169 , $59-64$

Munger KL, Levin LI, Hollis BW, Howard NS, Ascherio A (2006): Serum 25hydroxyvitamin D levels and risk of multiple sclerosis. JAMA 296 (23), 2832-2838

Myhr KM, Riise T, Barrett-Connor E, Myrmel H, Vedeler C, Grønning M, Kalvenes MB, Nyland H (1998): Altered antibody pattern to Epstein-Barr virus but not to other herpesviruses in multiple sclerosis: a population based case-control study from western Norway. J Neurol Neurosurg Psychiatr 64 (4), 539-542

Noseworthy JH, Lucchinetti C, Rodriguez M, Weinshenker BG (2000): Multiple sclerosis. N Engl J Med 343 (13), 938-952

Ontaneda D, Fox RJ, Chataway J (2015): Clinical trials in progressive multiple sclerosis: lessons learned and future perspectives. Lancet Neurol 14 (2), 208-223

Pache M, Kaiser HJ, Akhalbedashvili N, Lienert C, Dubler B, Kappos L, Flammer J (2003): Extraocular blood flow and endothelin-1 plasma levels in patients with multiple sclerosis. Eur Neurol $\underline{49}$ (3), 164-168

Papadaki EZ, Mastorodemos VC, Amanakis EZ, Tsekouras KC, Papadakis AE, Tsavalas ND, Simos PG, Karantanas AH, Plaitakis A, Maris TG (2012): White matter and 
deep gray matter hemodynamic changes in multiple sclerosis patients with clinically isolated syndrome. Magn Reson Med 68 (6), 1932-1942

Papadaki EZ, Simos PG, Mastorodemos VC, Panou T, Maris TG, Karantanas AH, Plaitakis A (2014a): Regional MRI perfusion measures predict motor/executive function in patients with clinically isolated syndrome. Behav Neurol 2014, 252419

Papadaki EZ, Simos PG, Panou T, Mastorodemos VC, Maris TG, Karantanas AH, Plaitakis A (2014b): Hemodynamic evidence linking cognitive deficits in clinically isolated syndrome to regional brain inflammation. Eur J Neurol 21 (3), 499-505

Peruzzo D, Castellaro M, Calabrese M, Veronese E, Rinaldi F, Bernardi V, Favaretto A, Gallo P, Bertoldo A (2012): Heterogeneity of cortical lesions in multiple sclerosis: an MRI perfusion study. J Cereb Blood Flow Metab 33 (3), 457-463

Peterson JW, Bø L, Mørk S, Chang A, Trapp BD (2001): Transected neurites, apoptotic neurons, and reduced inflammation in cortical multiple sclerosis lesions. Ann Neurol $50(3), 389-400$

Petzold GC, Murthy VN (2011): Role of astrocytes in neurovascular coupling. Neuron $71(5), 782-797$

Pittock SJ, Mayr WT, McClelland RL, Jorgensen NW, Weigand SD, Noseworthy JH, Rodriguez M (2004): Disability profile of MS did not change over 10 years in a population-based prevalence cohort. Neurology 62 (4), 601-606

Pohl D, Krone B, Rostasy K, Kahler E, Brunner E, Lehnert M, Wagner HJ, Gärtner J, Hanefeld F (2006): High seroprevalence of Epstein-Barr virus in children with multiple sclerosis. Neurology 67 (11), 2063-2065

Polman CH, O’Connor PW, Havrdova E, Hutchinson M, Kappos L, Miller DH, Phillips JT, Lublin FD, Giovannoni G, Wajgt A (2006): A randomized, placebo-controlled trial of natalizumab for relapsing multiple sclerosis. N Engl J Med 354 (9), 899-910

Polman CH, Reingold SC, Banwell B, Clanet M, Cohen JA, Filippi M, Fujihara K, Havrdova E, Hutchinson M, Kappos L (2011): Diagnostic criteria for multiple sclerosis: 2010 revisions to the McDonald criteria. Ann Neurol 69 (2), 292-302 
Ponsonby AL, van der Mei I, Dwyer T, Blizzard L, Taylor B, Kemp A, Simmons R, Kilpatrick T (2005): Exposure to infant siblings during early life and risk of multiple sclerosis. JAMA 293 (4), 463-469

Popescu V, Agosta F, Hulst HE, Sluimer IC, Knol DL, Sormani MP, Enzinger C, Ropele S, Alonso J, Sastre-Garriga J (2013): Brain atrophy and lesion load predict long term disability in multiple sclerosis. J Neurol Neurosurg Psychiatry 84 (10), 1082-1091

Pugliatti M, Rosati G, Carton H, Riise T, Drulovic J, Vécsei L, Milanov I (2006): The epidemiology of multiple sclerosis in europe. Eur J Neurol 13 (7), 700-722

Ransohoff RM, Hafler DA, Lucchinetti CF (2015): Multiple sclerosis-a quiet revolution. Nat Rev Neurol 11 (3), 134-142

Rao SM, Leo GJ, Bernardin L, Unverzagt F (1991): Cognitive dysfunction in multiple sclerosis. I. frequency, patterns, and prediction. Neurology 41 (5), 685-691

Rashid W, Parkes LM, Ingle GT, Chard DT, Toosy AT, Altmann DR, Symms MR, Tofts PS, Thompson AJ, Miller DH (2004): Abnormalities of cerebral perfusion in multiple sclerosis. J Neurol Neurosurg Psychiatr 75 (9), 1288-1293

Raz E, Cercignani M, Sbardella E, Totaro P, Pozzilli C, Bozzali M, Pantano P (2010): Gray- and white-matter changes 1 year after first clinical episode of multiple sclerosis: MR imaging. Radiology 257 (2), 448-454

Reuter M, Rosas HD, Fischl B (2010): Highly accurate inverse consistent registration: A robust approach. NeuroImage 53 (4), 1181-1196

Reuter M, Schmansky NJ, Rosas HD, Fischl B (2012): Within-subject template estimation for unbiased longitudinal image analysis. NeuroImage 61 (4), 1402-1418

Robertson NP, Fraser M, Deans J, Clayton D, Walker N, Compston DA (1996): Ageadjusted recurrence risks for relatives of patients with multiple sclerosis. Brain $119(\mathrm{Pt}$ 2), 449-455

Rosset A, Spadola L, Ratib O (2004): OsiriX: an open-source software for navigating in multidimensional DICOM images. J Digit Imaging 17 (3), 205-216 
Rot U, Mesec A (2006): Clinical, MRI, CSF and electrophysiological findings in different stages of multiple sclerosis. Clin Neurol Neurosurg 108 (3), 271-274

Sadovnick AD, Baird PA, Ward RH (1988): Multiple sclerosis: updated risks for relatives. Am J Med Genet 29 (3), 533-541

Sailer M, Fischl B, Salat D, Tempelmann C, Schönfeld MA, Busa E, Bodammer N, Heinze HJ, Dale A (2003): Focal thinning of the cerebral cortex in multiple sclerosis. Brain 126 (Pt 8), 1734-1744

Saindane AM, Law M, Ge Y, Johnson G, Babb JS, Grossman RI (2007): Correlation of diffusion tensor and dynamic perfusion MR imaging metrics in normal-appearing corpus callosum: support for primary hypoperfusion in multiple sclerosis. AJNR Am J Neuroradiol 28 (4), 767-772

Sanfilipo MP, Benedict RHB, Sharma J, Weinstock-Guttman B, Bakshi R (2005): The relationship between whole brain volume and disability in multiple sclerosis: A comparison of normalized gray vs. white matter with misclassification correction. NeuroImage 26 (4), 1068-1077

Sanfilipo MP, Benedict RHB, Weinstock-Guttman B, Bakshi R (2006): Gray and white matter brain atrophy and neuropsychological impairment in multiple sclerosis. Neurology 66 (5), 685-692

Schmainda KM, Rand SD, Joseph AM, Lund R, Ward BD, Pathak AP, Ulmer JL, Badruddoja MA, Krouwer HGJ (2004): Characterization of a first-pass gradientecho spin-echo method to predict brain tumor grade and angiogenesis. AJNR Am J Neuroradiol 25 (9), 1524-1532

Schulz D, Kopp B, Kunkel A, Faiss JH (2006): Cognition in the early stage of multiple sclerosis. J Neurol 253 (8), 1002-1010

Seewann A, Vrenken H, van der Valk P, Blezer ELA, Knol DL, Castelijns JA, Polman CH, Pouwels PJW, Barkhof F, Geurts JJG (2009): Diffusely abnormal white matter in chronic multiple sclerosis: imaging and histopathologic analysis. Arch Neurol 66 (5), 601-609 
Ségonne F, Dale AM, Busa E, Glessner M, Salat D, Hahn HK, Fischl B (2004): A hybrid approach to the skull stripping problem in mri. NeuroImage 22 (3), 1060-1075

Ségonne F, Pacheco J, Fischl B (2007): Geometrically accurate topology-correction of cortical surfaces using nonseparating loops. IEEE Trans Med Imaging 26, 518-529

SIENAX (2016): http://fsl.fmrib.ox.ac.uk/fsl/fslwiki/SIENA\#Referencing_ SIENA.2FX_.28more_detailed_text_and_references.29; accessed on 24/11/2016

Silber E, Sharief MK (1999): Axonal degeneration in the pathogenesis of multiple sclerosis. J Neurol Sci 170 (1), 11-18

Simon JH (2006): Brain atrophy in multiple sclerosis: what we know and would like to know. Mult Scler 12 (6), 679-687

Simon KC, van der Mei IaF, Munger KL, Ponsonby A, Dickinson J, Dwyer T, Sundström P, Ascherio A (2010): Combined effects of smoking, anti-EBNA antibodies, and HLADRB1*1501 on multiple sclerosis risk. Neurology 74 (17), 1365-1371

Sinnecker T, Dörr J, Pfueller CF, Harms L, Ruprecht K, Jarius S, Brück W, Niendorf T, Wuerfel J, Paul F (2012a): Distinct lesion morphology at 7-T MRI differentiates neuromyelitis optica from multiple sclerosis. Neurology $\underline{79}$ (7), 708-714

Sinnecker T, Mittelstaedt P, Dörr J, Pfueller CF, Harms L, Niendorf T, Paul F, Wuerfel J (2012b): Multiple sclerosis lesions and irreversible brain tissue damage: a comparative ultrahigh-field strength magnetic resonance imaging study. Arch Neurol 69 (6), 739 745

Sinnecker T, Bozin I, Dörr J, Pfueller CF, Harms L, Niendorf T, Brandt AU, Paul F, Wuerfel J (2013): Periventricular venous density in multiple sclerosis is inversely associated with t2 lesion count: a 7 tesla MRI study. Mult Scler 19 (3), 316-325

Sled J, Zijdenbos A, Evans A (1998): A nonparametric method for automatic correction of intensity nonuniformity in mri data. IEEE Trans Med Imaging 17, 87-97

Smeets D, Ribbens A, Sima DM, Cambron M, Horakova D, Jain S, Maertens A, Van Vlierberghe E, Terzopoulos V, Van Binst AM (2016): Reliable measurements 
of brain atrophy in individual patients with multiple sclerosis. Brain Behav $\underline{6}$ (9), $\mathrm{e} 00518$

Smith SM (2002): Fast robust automated brain extraction. Hum Brain Mapp 17 (3), $143-155$

Smith SM, De Stefano N, Jenkinson M, Matthews PM (2001): Normalized accurate measurement of longitudinal brain change. J Comput Assist Tomogr 25 (3), 466-475

Smith SM, Zhang Y, Jenkinson M, Chen J, Matthews PM, Federico A, De Stefano N (2002): Accurate, robust, and automated longitudinal and cross-sectional brain change analysis. NeuroImage 17 (1), 479-489

Smith SM, Jenkinson M, Woolrich MW, Beckmann CF, Behrens TEJ, Johansen-Berg H, Bannister PR, De Luca M, Drobnjak I, Flitney DE (2004): Advances in functional and structural MR image analysis and implementation as FSL. NeuroImage 23 (Suppl 1), S208-219

Smith SM, Rao A, De Stefano N, Jenkinson M, Schott JM, Matthews PM, Fox NC (2007): Longitudinal and cross-sectional analysis of atrophy in Alzheimer's disease: cross-validation of BSI, SIENA and SIENAX. NeuroImage 36 (4), 1200-1206

Sormani MP, Rovaris M, Valsasina P, Wolinsky JS, Comi G, Filippi M (2004): Measurement error of two different techniques for brain atrophy assessment in multiple sclerosis. Neurology 62 (8), 1432-1434

Sormani MP, Arnold DL, De Stefano N (2014): Treatment effect on brain atrophy correlates with treatment effect on disability in multiple sclerosis. Ann Neurol 75 (1), $43-49$

Spach KM, Hayes CE (2005): Vitamin D3 confers protection from autoimmune encephalomyelitis only in female mice. J Immunol 175 (6), 4119-4126

Speciale L, Sarasella M, Ruzzante S, Caputo D, Mancuso R, Calvo MG, Guerini FR, Ferrante P (2000): Endothelin and nitric oxide levels in cerebrospinal fluid of patients with multiple sclerosis. J Neurovirol 6 (Suppl 2), S62-66 
Steen C, D'haeseleer M, Hoogduin JM, Fierens Y, Cambron M, Mostert JP, Heersema DJ, Koch MW, De Keyser J (2013): Cerebral white matter blood flow and energy metabolism in multiple sclerosis. Mult Scler 19 (10), 1282-1289

Sumaya CV, Myers LW, Ellison GW, Ench Y (1985): Increased prevalence and titer of Epstein-Barr virus antibodies in patients with multiple sclerosis. Ann Neurol 17 (4), $371-377$

Sundström P, Juto P, Wadell G, Hallmans G, Svenningsson A, Nyström L, Dillner J, Forsgren L (2004): An altered immune response to Epstein-Barr virus in multiple sclerosis: a prospective study. Neurology $\underline{62}$ (12), 2277-2282

Sundström P, Wahlin A, Ambarki K, Birgander R, Eklund A, Malm J (2010): Venous and cerebrospinal fluid flow in multiple sclerosis: a case-control study. Ann Neurol $\underline{68}(2), 255-259$

Swanton JK, Fernando K, Dalton CM, Miszkiel KA, Thompson AJ, Plant GT, Miller DH (2006): Modification of MRI criteria for multiple sclerosis in patients with clinically isolated syndromes. J Neurol Neurosurg Psychiatr 77 (7), 830-833

Swanton JK, Rovira A, Tintore M, Altmann DR, Barkhof F, Filippi M, Huerga E, Miszkiel KA, Plant GT, Polman C (2007): MRI criteria for multiple sclerosis in patients presenting with clinically isolated syndromes: a multicentre retrospective study. Lancet Neurol $\underline{6}$ (8), 677-686

Tallantyre EC, Brookes MJ, Dixon JE, Morgan PS, Evangelou N, Morris PG (2008): Demonstrating the perivascular distribution of MS lesions in vivo with 7-Tesla MRI. Neurology $\underline{70}$ (22), 2076-2078

Tallantyre EC, Morgan PS, Dixon JE, Al-Radaideh A, Brookes MJ, Evangelou N, Morris PG (2009): A comparison of $3 \mathrm{~T}$ and $7 \mathrm{~T}$ in the detection of small parenchymal veins within MS lesions. Invest Radiol 44 (9), 491-494

Tallantyre EC, Dixon JE, Donaldson I, Owens T, Morgan PS, Morris PG, Evangelou N (2011): Ultra-high-field imaging distinguishes MS lesions from asymptomatic white matter lesions. Neurology $\underline{76}$ (6), 534-539 
Tanaka R, Iwasaki Y, Koprowski H (1975): Ultrastructural studies of perivascular cuffing cells in multiple sclerosis brain. Am J Pathol 81 (3), 467-478

Tedeholm H, Lycke J, Skoog B, Lisovskaja V, Hillert J, Dahle C, Fagius J, Fredrikson S, Landtblom AM, Malmeström C (2013): Time to secondary progression in patients with multiple sclerosis who were treated with first generation immunomodulating drugs. Mult Scler 19 (6), 765-774

Tedeschi G, Lavorgna L, Russo P, Prinster A, Dinacci D, Savettieri G, Quattrone A, Livrea P, Messina C, Reggio A (2005): Brain atrophy and lesion load in a large population of patients with multiple sclerosis. Neurology 65 (2), 280-285

Thacker EL, Mirzaei F, Ascherio A (2006): Infectious mononucleosis and risk for multiple sclerosis: a meta-analysis. Ann Neurol 59 (3), 499-503

Trapp BD, Nave KA (2008): Multiple sclerosis: an immune or neurodegenerative disorder? Ann Rev Neurosci 31, 247-269

Trapp BD, Peterson J, Ransohoff RM, Rudick R, Mörk S, Bö L (1998): Axonal transection in the lesions of multiple sclerosis. N Engl J Med 338 (5), 278-285

Tsivgoulis G, Faissner S, Voumvourakis K, Katsanos AH, Triantafyllou N, Grigoriadis N, Gold R, Krogias C (2015): "Liberation treatment" for chronic cerebrospinal venous insufficiency in multiple sclerosis: the truth will set you free. Brain Behav $\underline{5}$ (1), 3-12

Tsunoda I, Tanaka T, Saijoh Y, Fujinami RS (2007): Targeting inflammatory demyelinating lesions to sites of Wallerian degeneration. Am J Pathol 171 (5), 1563-1575

Valdueza JM, Doepp F, Schreiber SJ, van Oosten BW, Schmierer K, Paul F, Wattjes MP (2013): What went wrong? The flawed concept of cerebrospinal venous insufficiency. J Cereb Blood Flow Metab 33 (5), 657-668

van Osch MJP, Vonken EJPA, Wu O, Viergever MA, van der Grond J, Bakker CJG (2003): Model of the human vasculature for studying the influence of contrast injection speed on cerebral perfusion MRI. Magn Reson Med 50 (3), 614-622

Varga AW, Johnson G, Babb JS, Herbert J, Grossman RI, Inglese M (2009): White matter hemodynamic abnormalities precede sub-cortical gray matter changes in multiple sclerosis. J Neurol Sci 282 (1), 28-33 
Vollmer T, Signorovitch J, Huynh L, Galebach P, Kelley C, DiBernardo A, Sasane R (2015): The natural history of brain volume loss among patients with multiple sclerosis: a systematic literature review and meta-analysis. J Neurol Sci 357 (1-2), $8-18$

Vucic S, Burke T, Lenton K, Ramanathan S, Gomes L, Yannikas C, Kiernan MC (2012): Cortical dysfunction underlies disability in multiple sclerosis. Mult Scler 18 (4), 425432

Wagner HJ, Hennig H, Jabs WJ, Siekhaus A, Wessel K, Wandinger KP (2000): Altered prevalence and reactivity of anti-Epstein-Barr virus antibodies in patients with multiple sclerosis. Viral Immunol $\underline{13}$ (4), 497-502

Wattjes MP, Doepp F, Bendszus M, Fiehler J (2011a): "Chronic cerebrospinal venous insufficiency" in multiple sclerosis - is multiple sclerosis a disease of the cerebrospinal venous outflow system? RoFo 183 (6), 523-530

Wattjes MP, van Oosten BW, de Graaf WL, Seewann A, Bot JCJ, van den Berg R, Uitdehaag BMJ, Polman CH, Barkhof F (2011b): No association of abnormal cranial venous drainage with multiple sclerosis: a magnetic resonance venography and flowquantification study. J Neurol Neurosurg Psychiatr 82 (4), 429-435

Whitacre CC (2001): Sex differences in autoimmune disease. Nat Immunol 2 (9), $777-$ 780

Wuerfel J, Bellmann-Strobl J, Brunecker P, Aktas O, McFarland H, Villringer A, Zipp F (2004): Changes in cerebral perfusion precede plaque formation in multiple sclerosis: a longitudinal perfusion MRI study. Brain 127, 111-119

Wuerfel J, Paul F, Zipp F (2007): Cerebral blood perfusion changes in multiple sclerosis. J Neurol Sci 259 (1), 16-20

Wuerfel J, Haertle M, Waiczies H, Tysiak E, Bechmann I, Wernecke KD, Zipp F, Paul F (2008): Perivascular spaces-MRI marker of inflammatory activity in the brain? Brain 131 (Pt 9), 2332-2340 
Wuerfel J, Sinnecker T, Ringelstein EB, Jarius S, Schwindt W, Niendorf T, Paul F, Kleffner I, Dörr J (2012): Lesion morphology at 7 Tesla MRI differentiates Susac syndrome from multiple sclerosis. Mult Scler 18 (11), 1592-1599

Zamboni P (2006): The big idea: iron-dependent inflammation in venous disease and proposed parallels in multiple sclerosis. J R Soc Med 99 (11), 589-593

Zamboni P, Galeotti R (2010): The chronic cerebrospinal venous insufficiency syndrome. Phlebology 25 (6), 269-279

Zamboni P, Menegatti E, Bartolomei I, Galeotti R, Malagoni AM, Tacconi G, Salvi F (2007): Intracranial venous haemodynamics in multiple sclerosis. Curr Neurovasc Res $\underline{4}(4), 252-258$

Zamboni P, Galeotti R, Menegatti E, Malagoni AM, Gianesini S, Bartolomei I, Mascoli F, Salvi F (2009a): A prospective open-label study of endovascular treatment of chronic cerebrospinal venous insufficiency. J Vasc Surg 50 (6), 1348-1358

Zamboni P, Galeotti R, Menegatti E, Malagoni AM, Tacconi G, Dall'Ara S, Bartolomei I, Salvi F (2009b): Chronic cerebrospinal venous insufficiency in patients with multiple sclerosis. J Neurol Neurosurg Psychiatr 80 (4), 392-399

Zamboni P, Menegatti E, Galeotti R, Malagoni AM, Tacconi G, Dall'Ara S, Bartolomei I, Salvi F (2009c): The value of cerebral doppler venous haemodynamics in the assessment of multiple sclerosis. J Neurol Sci 282 (1), 21-27

Zhang Y, Brady M, Smith S (2001): Segmentation of brain MR images through a hidden markov random field model and the expectation-maximization algorithm. IEEE Trans Med Imaging 20 (1), 45-57

Zipp F (2009): A new window in multiple sclerosis pathology: non-conventional quantitative magnetic resonance imaging outcomes. J Neurol Sci 287 (Suppl 1), S24-29

Zivadinov R, Bakshi R (2004): Central nervous system atrophy and clinical status in multiple sclerosis. J Neuroimaging 14 (3 Suppl), 27S-35S

Zivadinov R, Reder AT, Filippi M, Minagar A, Stüve O, Lassmann H, Racke MK, Dwyer MG, Frohman EM, Khan O (2008): Mechanisms of action of disease-modifying agents and brain volume changes in multiple sclerosis. Neurology 71 (2), 136-144 
Zivadinov R, Weinstock-Guttman B, Hashmi K, Abdelrahman N, Stosic M, Dwyer M, Hussein S, Durfee J, Ramanathan M (2009): Smoking is associated with increased lesion volumes and brain atrophy in multiple sclerosis. Neurology 73 (7), 504-510

Zivadinov R, Lopez-Soriano A, Weinstock-Guttman B, Schirda CV, Magnano CR, Dolic K, Kennedy CL, Brooks CL, Reuther JA, Hunt K (2011): Use of MR venography for characterization of the extracranial venous system in patients with multiple sclerosis and healthy control subjects. Radiology 258 (2), 562-570

Zivadinov R, Bergsland N, Dolezal O, Hussein S, Seidl Z, Dwyer MG, Vaneckova M, Krasensky J, Potts JA, Kalincik T (2013a): Evolution of cortical and thalamus atrophy and disability progression in early relapsing-remitting MS during 5 years. AJNR Am J Neuroradiol 34 (10), 1931-1939

Zivadinov R, Havrdová E, Bergsland N, Tyblova M, Hagemeier J, Seidl Z, Dwyer MG, Vaneckova M, Krasensky J, Carl E (2013b): Thalamic Atrophy Is Associated with Development of Clinically Definite Multiple Sclerosis. Radiology 268 (3), 831-841 


\section{Acknowledgements}

I would like to express my gratitude and appreciation to Prof. Dr. med. Michael Knauth for giving me the opportunity to work on this project in his department and for his supervision of this thesis.

I would like to thank Dr. med. Jens Würfel for suggesting this project and for his scientific advice.

I am grateful to Dr. med. Philipp von Gottberg for his help regarding the quality assessment of the raw data. 